\title{
BOOTSTRAP NÃO-PARAMÉTRICO \\ APLICADO A DADOS INCOMPLETOS
}

\section{Delhi Teresä Paiva Salinas}

DISSERTAÇÃO APRESENTADA

$\mathrm{AO}$

INSTITUTO DE MATEMÁTICA E ESTATÍSTICA

DA

UNIVERSIDADE DE SÃO PAULO

PARA OBTENÇÃO DO GRAU

DE

MESTRE EM ESTATÍSTICA

Área de Concentração: Estatística

Orientadora: Profa. Dra. Lúcia Pereira Barroso

-São Paulo, julho de 1998- 
Este exemplar corresponde à redação final da dissertação devidamente corrigida e defendida por Delhi Teresa Paiva Salinas e aprovada pela comissão julgadora.

São Paulo, 23 de julho.

Banco Examinadora:

- Profa. Dra. Lúcia Pereira Barroso (Orientadora) - IME - USP

- Profa. Dra. Carmen Diva Saldiva de André - IME - USP

- Prof. Dr. Francisco Cribari Neto - UFPE 
A Deus, pela oportunidade de concluir mais uma etapa de minha vida, graças a tua força e sabedoria infinita.

À meus pais, Luis e Vilma meus irmãos Carmen e Luigy, por todo o amor, compreensão apoio e estímulo. À memoria de minha avó Hibertia por ter acreditado sempre em mim. 


\section{Agradecimentos}

Gostaria de registrar meu agradecimento às pessoas que, de uma forma ou outra, participaram do processo de elaboração deste trabalho.

Em especial, agradeço à Profa. Dra. Lúcia Pereira Barroso, que sugeriu o tema e orientou este trabalho e que me dispensou enorme dedicação e atenção estando sempre disponivel nos momentos em que precisei. Também agradeço aos demais professores do IME- ¿SP, que foram responsáveis pela minha formação.

Além disso, foi de enorme valia o auxílio contínuo fornecido por Adriano, José, Rosane e Eliana, pela força e amizade. Destaco Vicente e Beto dentre as pessoas que me auxiliaram a usar os computadores no uso do LATEX. À Katia, Sumaia, Miriam e Jô pela amizade, paciência, apoio e imensa solidariedade tão fundamentais neste período. Aos demais colegas e amigos que se fizeram presentes sob o aspecto da amizade, do incentivo e do apoio, o meu muito obrigado.

Desejo registrar meu agradecimento a Dona Benê, Paulo e Alê, pela oportunidade de sentir-me em família permitindo que minha estadia no Brasil fosse mais agradável.

Finalmente à minha família pela força e amor sempre presentes, por ter acreditado em minha capacidade de concluir este trabalho e por ter participado dos meus dias mais difícieis durante esse período. Obrigada por saber que posso contar com vocês.

Também agradeço ao CNPq e CAPES pela verba dispensada, sem a qual este projeto não seria possivel. 


\section{Resumo}

Um problema bastante comum em levantamentos amostrais é a falta de algumas das informações. Uma maneira de tratar esse problema é a imputação, que consiste em prever as observações perdidas, completando o conjunto de dados para depois analisá-lo como se fosse completo.

Em particular, essa idéia também pode ser aplicada quando se pretende utilizar o método bootstrap para se estimar o erro padrão de algum estimador. Apesar dessa técnica ser bastante utilizada atualmente, pouca atenção tem sido dada à sua aplicação na ausência de informações.

Este trabalho discorre sobre a aplicação da técnica bootstrap não-paramétrica a dados incompletos, caso em que o procedimento usual é aplicá-la em conjunto com algum método de imputação. Apresentamos os resultados de simulação sobre a estimação da variância dos estimadores das componentes da variância em um modelo linear misto e mediante a geração de dados através de uma distribuição normal bivariada. As amostras utilizadas são do tipo painel onde $n$ indivíduos foram gerados em dois instantes de tempo. 


\section{Abstract}

A commom problem in sampling surveys is the missing of some data. One way to handle this problem is using imputation techniques, which consist of predicting the missing observations to complete the data set which will be later analised.

Imputation techniques can be also applied when we want to use the bootstrap method to estimate the standard error of some estimator. Although that techniques has been widely utilized nowadays, it has not received much attention for data with missing values.

The main objetive in this work is the application of the non-parametric bootstrap techniques for data with missing values with imputation techniques to replace missing values.

We present the results of simulation about the estimation of the variance of the variance components estimators in a mixed linear model, and through of the generation data from normal bivariate distribution. The samples are panels, where $n$ individuals have been observed in two ocassions. 


\section{Sumário}

1 Introdução 1

2 A técnica bootstrap 3

2.1 Introdução . . . . . . . . . . . . . . . . . . 3

2.2 o bootstrap . . . . . . . . . . . . . . . . 5

2.2 .1 O bootstrap não paramétrico do erro padrão . . . . . . . . . 5

2.2 .2 Tipos de bootstrap . . . . . . . . . . . . . 7

2.3 Estimador bootstrap do vício . . . . . . . . . . . . . 10

2.4 Intervalos de confiança . . . . . . . . . . . . . . . . 11

2.4 .1 Intervalos bootstrap-t . . . . . . . . . . . . . 12

2.4 .2 Intervalos bootstrap-p . . . . . . . . . . . . . 14

2.5 Ilustração numérica . . . . . . . . . . . . . . . . . . 16

3 Imputação de dados $\quad 21$

3.1 Introdução . . . . . . . . . . . . . . . . . . . . 21

3.2 BLUP para o modelo misto . . . . . . . . . . . . . . . 23

3.2 .1 O método de imputação . . . . . . . . . . . . . . . . . . 28

3.2 .2 Método de estimação das componentes da variância . . . . . . . . 29

3.3 Método de Substituição da Média (MSM) . . . . . . . . . . . . . 33

3.4 Método de Componentes Principais de Dear (DPC) . . . . . . . . . 37 
3.5 O algoritmo EM . . . . . . . . . . . . . . . 41

3.5 .1 Descrição do algoritmo . . . . . . . . . . . . . . . . 42

4 O bootstrap aplicado a dados incompletos $\quad 48$

4.1 Introdução . . . . . . . . . . . . . . . . . . . . . . . . 48

4.2 Algoritmo bootstrap para dados incompletos . . . . . . . . . . 50

4.3 Resultados da simulação . . . . . . . . . . . . . . . . . 51

$\begin{array}{ll}\text { Conclusões } & 74\end{array}$

Apêndice $\quad 76$

$\begin{array}{ll}\text { Referências bibliográficas } & 98\end{array}$ 


\section{Capítulo 1}

\section{Introdução}

A utilização de técnicas de reamostragem tem tido um importante papel na Estatística, especialmente quando o cálculo de estimadores por métodos analíticos é complicado. Uma delas, a técnica bootstrap, foi introduzida por Efron (1979) e teve grande desenvolvimento nos últimos quinze anos, mostrando-se uma interessante ferramenta de estimação.

A idéia é reamostrar as observações de forma adequada, sorteando réplicas da amostra original para com elas estimar, por exemplo, o vício e o erro padrão de algum estimador.

Como essa técnica é relativamente nova, apesar da vasta bibliografia já existente, pouca atenção tem sido dada à sua aplicação a dados incompletos. A falta de dados em levantamentos amostrais é uma situação bastante frequente e uma maneira de tratar o problema é utilizar algum método de imputação, que consiste em preencher o conjunto de dados com previsões individuais para depois analisá-lo como se fosse completo.

O objetivo deste trabalho é descrever a técnica bootstrap não-paramétrica aplicada a dados com informações ausentes. A técnica usada em conjunto com algum método de imputação é descrita de uma maneira geral e é aplicada a um painel fixo, onde uma amostra é selecionada em um primeiro instante, tendo depois seus dados coletados para várias ocasiões.

Este trabalho está dividido em quatro capítulos. No Capítulo 2, descrevemos o bo- 
otstrap para dados completos, com especial atenção ao bootstrap não paramétrico do erro padrão e vício. No Capítulo 3 , mostramos quatro diferentes métodos de imputação determinísticos, escolhidos arbitrariamente. São eles o BLUP (Best Linear Unbiased Predictor), sustituição da média (MSM), componentes principais de Dear (DPC) e o algoritmo EM, cada um deles com exemplos numéricos. No Capítulo 4, introduzimos o bootstrap não-paramétrico aplicado a dados incompletos, apresentando o algoritmo modificado e os resultados da simulação feita no S-PLUS, cujo programa encontra-se no Apêndice deste trabalho. Esta simulação foi feita para estimar as variâncias dos estimadores das componentes de variância em um modelo linear misto com os dados gerados sob o modelo e sob a distribuição normal bivariada. 


\section{Capítulo 2}

\section{A técnica bootstrap}

\subsection{Introdução}

Neste capítulo descrevemos uma das técnicas de reamostragem, o bootstrap, introduzida por Efron (1979). As técnicas de reamostragem são ferramentas versáteis baseadas no uso de um conjunto de dados repetidas vezes. O bootstrap é uma técnica computacional utilizada frequentemente para medir o vício ou o erro padrão de um estimador. Ele é particularmente útil quando não se conhece a forma explícita do erro padrão do estimador.

Esta técnica procura reproduzir, a partir de uma amostra, o mecanismo de geração de dados, evitando a derivação de fórmulas complicadas.

O bootstrap foi introduzido na forma não paramétrica, que é a mais comumente utilizada, mas uma versão paramétrica do mesmo foi sugerida posteriormente. Efron (1979, 1982 ) consideram uma variedade de problemas estatísticos e mostram a facilidade da aplicação desse método de simulaçào. As contribuições particulares de Efron $(1982,1990)$ são a realização da simulação sem modelos paramétricos e o relacionamento dos métodos de simulação com as técnicas de jackknife e validação cruzada.

O jackknife e o bootstrap usual são métodos não paramétricos cujas vantagens sobre 
a aproximação paramétrica tradicional é que são fáceis de descrever e aplicam-se arbitrariamente a situações complicadas, não sendo necessário fazer suposições sobre a forma da distribuição da população.

Freedman (1981) e Wu (1986) proporcionam uma discussão detalhada sobre propriedades do bootstrap em análises de regressão, tratando de dois problemas, o primeiro, determinar a exatidão dos coeficientes de regressão ou valores ajustados da resposta média, e o segundo estudar a seleção de variáveis ou do modelo sobre o vício de alguma medida do modelo ajustado. No caso multivariado, Beran e Srivastava (1985) apresentam resultados do bootstrap para a matriz de covariâncias. Efron e Tibshirani (1986) discutem muitos exemplos interessiantes da aplicação do bootstrap para procedimentos estatísticos complicados tais como séries de tempo e dados censurados. Fisher e Hall (1989) mostram como obter regiões de confiança via bootstrap quando se trabalha com dados circulares. Mais recentemente, Manteiga, Sanchez e Romo (1994) apresentam as principais idéias da metodologia em vários contextos, Young (1994) faz uma revisão crítica do mesmo com a discussão de vários pesquisadores e Frangos e Schucany (1995) comparam o bootstrap, o jackknife e métodos clássicos em um experimento animal, através de intervalos de confiança quando o parâmetro de interesse é a proporção. Uma importante referência sobre o bootstrap é Efron e Tibshirani (1993), que traz uma vasta bibliografia sobre o assunto.

A técnica bootstrap tem também uma versão Bayesiana, chamada bootstrap Bayesiano, introduzida por Rubin (1981). Lo (1987) introduziu o bootstrap Bayesiano para populações finitas e discutiu as propriedades das distribuições para pequenas amostras. Ele mostrou que para uma variedade de funcionais, o bootstrap Bayesiano e o bootstrap para populações finitas são assintoticamente equivalentes de primeira ordem. 


\subsection{O bootstrap}

Um problema comum em estatística é a estimação de um parâmetro desconhecido $\theta$. Daquí surgem duas questões.

1. Qual é o estimador $\hat{\theta}$ que será usado?

2. Tendo escolhido $\hat{\theta}$, quão exato é $\hat{\theta}$ como um estimador de $\theta$ ?

Uma medida bastante utilizada para responder à segunda questão é o erro padrão do estimador, cuja expressão é obtida pela Teoria Estatística e muitas vezes é conhecida. Entretanto há situações em que a expressão do erro padrão é desconhecida e, nesse caso, o bootstrap ajudaria a responder essa questão.

Os métodos bootstrap são mais usados em problemas complexos, onde o trabalho teórico proporciona aproximaçoes não exatas, nem robustas. É visto como uma ferramenta geral que pode ser usada para abordar uma ampla variedade de problemas estatísticos, proporcionando usualmente um procedimento estatístico consistente quando os dados são independentes.

\subsubsection{O bootstrap não paramétrico do erro padrão}

Para introduzir a idéia do bootstrap descrevemos primeiro um caso de interesse, o bootstrap não paramétrico do erro padrão, para depois tratarmos dos tipos de bootstrap.

Seja $\mathbf{y}=\left(y_{1}, y_{2}, \ldots, y_{n}\right)$ uma amostra aleatória de variáveis aleatórias independentes e identicamente distribuídas, com função de distribuição comum $F$, isto é, a partir de uma distribuição de probabilidade $F$, selecionamos uma amostra de tamanho $n, F \longrightarrow$ $\mathrm{y}=\left(y_{1}, y_{2}, \ldots, y_{n}\right)$. Seja $\hat{F}_{n}$ a função de distribuição empírica de $\mathrm{y}$, ou seja, a função que associa a cada $y_{i}, i=1,2, \ldots, n$, a probabilidade $1 / n$. Uma amostra bootstrap é definida como uma amostra aleatória com reposição de tamanho $m$, selecionada de $\hat{F}_{n}$. Usualmente, sorteia-se a amostra bootstrap de mesmo tamanho da amostra original. ou 
seja, $m=n$, mas isso não é necessário. Há também possibilidade de se fazer o sorteio sem reposição. Neste caso o tamanho das amostras bootstrap tem que ser no máximo $n-1$ (ver Sitter (1992)). Neste trabalho adotamos tamanho igual, esquematizado por $\hat{F}_{n} \longrightarrow \mathrm{y}^{*}=\left(y_{1}^{*}, y_{2}^{*}, \ldots, y_{n}^{*}\right)$.

Uma outra forma de dizer isto é que os dados bootstrap $y_{1}^{*}, y_{2}^{*}, \ldots, y_{n}^{*}$ são uma amostra aleatória de dimensão $n$ selecionada com reposição de uma população de $n$ objetos. Podemos ter, por exemplo, que uma das amostras bootstrap seja formada por $y_{1}^{*}=y_{4}, y_{2}^{*}=y_{1}, y_{3}^{*}=y_{29}, y_{4}^{*}=y_{1}, \ldots, y_{n}^{*}=y_{8}$. Assim, dizemos que um conjunto de dados bootstrap, $y_{1}^{*}, y_{2}^{*}, \ldots, y_{n}^{*}$, é formado por elementos do conjunto de dados originais $y_{1}, y_{2}, \ldots, y_{n}$ com cada ponto podendo ser sorteado $0,1,2$ ou mais vezes.

Seja $\theta=t(F)$ um parâmetro escrito diretamente como função de $F$, o que indica que o valor de $\theta$ é obtido aplicando-se algum procedimento de avaliação numérica $t(\cdot)$ à função de distribuição $F$. Um estimador de $\theta$ é $\hat{\theta}=s(\mathrm{y})$, podendo ter a mesma forma de $t$ aplicada à amostra ou não. Se a forma é a mesma, $\hat{\theta}=t\left(\hat{F}_{n}\right)$, diz-se que foi aplicado o princípio plug-in. Os estimadores plug-in apresentam em geral bom resultado se toda a informação vem da amostra. Quando há outra fonte de informação, como por exemplo sabe-se que a distribuição é normal, os resultados podem não ser tão bons.

Segundo Efron e Tibishirani (1993), estimadores plug-in não são necessariamente não viesados mas tendem a ter vício pequeno comparado com a magnitude do seu erro padrão.

Uma réplica bootstrap de $\hat{\theta} \cdot \hat{\theta}^{*}=s^{*}(\mathbf{y})$, é obtida aplicando-se a mesma função $s(\cdot)$ a $\mathrm{y}^{*}$. O estimador bootstrap não paramétrico usa a função de distribuição empírica $\hat{F}_{n}$ no lugar da distribuição desconhecida $F$. O estimador bootstrap não paramétrico do erro padrão de $\hat{\theta}$ é o estimador plug-in baseado nas réplicas $\hat{\theta}^{*}$, obtido pelo algoritmo bootstrap, descrito a seguir:

1. selecionar $B$ amostras bootstrap independentes, com reposição de $\mathbf{y}=\left(y_{1}, y_{2}, \ldots, y_{n}\right)$ $\mathrm{y}^{* 1}, \mathrm{y}^{* 2}, \ldots, \mathrm{y}^{* B}$, cada uma de dimensão $n$; 
2. calcular a réplica de $\hat{\theta}$ de cada amostra bootstrap

$$
\hat{\theta}^{*}(b)=s\left(\mathbf{y}^{* b}\right), \quad b=1,2, \ldots, B
$$

3. estimar o erro padrão de $\hat{\theta}, e p_{F}(\hat{\theta})$, pelo erro padrão amostral das $B$ réplicas.

$$
\hat{e p_{B}}=\left[\frac{1}{B-1} \sum_{b=1}^{B}\left(\hat{\theta}^{*}(b)-\hat{\theta}^{*}(\cdot)\right)^{2}\right]^{1 / 2},
$$

onde

$$
\hat{\theta}^{*}(\cdot)=\frac{1}{B} \sum_{b=1}^{B} \hat{\theta}^{*}(b) .
$$

Note que com as $B$ réplicas de $\hat{\theta}$ estima-se a distribuição do estimador $\hat{\theta}$. Segundo Efron e Tibshirani (1993), $B=50$ réplicas já é suficiente para a obtenção de uma boa estimativa do erro padrão ou do vício de um estimador. Dificilmente mais do que 200 réplicas são necessárias.

Muitos aplicativos computacionais podem ser utilizados para desenvolver esta técnica. Dentre eles tem-se o MINITAB, S-PLUS, SAS, OX, sendo o mais usado atualmente, o S-PLUS, que também será utilizado neste trabalho.

\subsubsection{Tipos de bootstrap}

Três são os tipos de bootstrap: paramétrico, não paramétrico e Bayesiano. A diferença entre eles é a maneira como as amostras são selecionadas.

Num problema amostral, em geral os dados $y_{1}, y_{2}, \ldots, y_{n}$ são independentes e identicamente distribuídos de acordo com uma distribuição $F$, isto é, a distribuição conjunta de $y_{1}, y_{2}, \ldots, y_{n}$ é determinada por $F$. Em consequência, se $F$ pertence a uma família paramétrica, então, $F=F_{\theta}$, onde $\theta$ é um vetor de parâmetros desconhecidos. Neste caso, $\theta$ é estimado por $\hat{\theta}$. 
Se a forma de $F$ é conhecida, e neste caso estamos falando do bootstrap paramétrico, $F_{\theta}$ é estimada por $F_{\hat{\theta}}$. Por outro lado, no caso do bootstrap não paramétrico, quando a forma de $F$ não é conhecida, $F$ pode ser estimada por $\hat{F}_{n}$, a distribuição empírica, definida como uma distribuição discreta que atribui probabilidade $\frac{1}{n}$ para cada $y_{i}, i=1,2, \ldots, n$.

No caso paramétrico, o conjunto de dados bootstrap $y_{1}^{*}, y_{2}^{*}, \ldots, y_{n}^{*}$ é gerado a partir de $F_{\dot{\theta}}$; no não paramétrico, a partir de $\hat{F}_{n}$, com amostragem aleatória simples com reposição. Note que o bootstrap não paramétrico pode ser usado tanto no modelo paramétrico como no não paramétrico. O bootstrap paramétrico depende das suposições do modelo paramétrico, enquanto que o bootstrap não paramétrico é um modelo de suposições livres, embora não seja tão eficiente quando o modelo paramétrico é correto.

Considere $\theta$ um elemento do vetor de parâmetros $\theta$. O algoritmo para estimar o erro padrão no caso paramétrico é similar ao não paramétrico, a diferença está no primeiro passo, na geração das amostras bootstrap. No caso da estimação do erro padrão de $\hat{\theta}$, o primeiro passo do algoritmo dado na página 6 , é substituído por:

1A estimar $\theta$ com base na amostra original $y=\left(y_{1}, y_{2}, \ldots, y_{n}\right)$;

1B gerar $B$ amostras bootstrap independentes $\mathrm{y}^{* 1}, \mathrm{y}^{* 2}, \ldots, \mathrm{y}^{* \mathrm{~B}}$, cada uma de dimensão $n$, da distribuição $F_{\hat{\theta}}$.

Os passos 2 e 3 permanecem os mesmos.

Os estimadores bootstrap raramente podem ser expressos em forma fechada; na maioria das vezes as simulações de Monte Carlo são usadas para suas aproximações.

O bootstrap Bayesiano é análogo ao bootstrap não paramétrico, somente substitui o tamanho dos saltos da função de distribuição empírica pelas diferenças de $n-1$ variáveis aleatórias independentes e identicamente distribuídas, segundo uma distribuição uniforme no intervalo $(0,1)$.

No não paramétrico, em cada replicação, a distribuição empírica atribui a $y_{i}$ como probabilidade, a proporção de vezes que $y_{i}$ aparece na amostra. Se denotarmos essa 
proporção por $f_{i}$ e se, por exemplo, estivermos interessados em estimar a média, teríamos $\hat{\theta}=\sum_{i=1}^{n} f_{i} y_{i}$. A proporção $f_{i}$ pode assumir valores $0, \frac{1}{n}, \frac{2}{n}, \ldots, \frac{n}{n}$.

Cada réplica do bootstrap Bayesiano gera uma probabilidade a posteriori para cada $y_{i}$, onde valores de y não observados têm probabilidade a posteriori igual a zero. A probabilidade a posteriori de cada um dos $y_{i}$ é centrada em $\frac{1}{n}$, e cada réplica consiste em gerar $n-1$ variáveis aleatórias uniformes $(0,1), U_{1}, \ldots, U_{n-1}$, ordená-las e calcular as diferenças $g_{i}=U_{(i)}-U_{(i-1)}, i=1,2, \ldots, n$, onde $U_{(0)}=0$ e $U_{(n)}=1$. Assim, $g=\left(g_{1}, \ldots, g_{n}\right)$ é o vetor de probabilidades associado aos valores $y_{1}^{*}, \ldots, y_{n}^{*}$ na réplica bootstrap. Se o interesse é estimar a média, $\hat{\theta}=\sum_{i=1}^{n} g_{i} y_{i}$. Considerando todas as réplicas obtém-se a distribuição bootstrap Bayesiana de qualquer parâmetro de interesse.

Assim, seja y uma amostra fixa. O algoritmo do bootstrap Bayesiano é dado por:

1. gerar $n-1$ variáveis aleátorias independentes e identicamente distribuídas segundo $U(0,1)$ independentes de $\mathrm{y}$. Ordenar os valores obtidos de modo que $0=U_{(0)}<$ $U_{(1)}<\ldots<U_{(n-1)}<U_{(n)}=1$. Seja $g_{i}=U_{(i)}-U_{(i-1)}, i=1,2, \ldots, n$ as $n$ diferenças dos consecutivos $U_{(i)}$ 's. Construir uma função de distribuição discreta aleátoria $D_{n}$ com pesos $g_{i}$ em $y_{i}$ para $i=1,2, \ldots, n$;

2. repetir o passo $1, B$ vezes, obtendo $D_{n 1}, D_{n 2}, \ldots, D_{n B}$ e calcular $\theta\left(D_{n 1}, \mathbf{y}\right), \ldots$, $\theta\left(D_{n B}, \mathrm{y}\right)$, denotado por $\hat{\theta}_{1}^{*}, \ldots, \hat{\theta}_{B}^{*}$, respectivamente;

3. a função distribuição empírica a posteriori de $\theta$ atribui $\frac{1}{B}$ para cada $\hat{\theta}_{j}^{*}$, para $j=$ $1,2, \ldots, B$

A interpretação da distribuição empírica resultante dos métodos Bayesianos e dos anteriores é diferente. No Bayesiano ela é interpretada como a distribuição a posteriori do parâmetro $\theta$ enquanto que nos anteriores como a distibuição do estimador $\hat{\theta}$.

Neste trabalho, nos deteremos na discussão do bootstrap não paramétrico, uma vez que é o mais utilizado. A partir de agora nos referiremos a ele simplesmente como bootstrap, que aliás é o que também ocorre na literatura. 


\subsection{Estimador bootstrap do vício}

$\mathrm{O}$ vício de qualquer estimador $\hat{\theta}=s(\mathrm{y})$ é definido pela diferença entre a esperança de $\hat{\theta}$ e o valor do parâmetro $\theta=t(F)$, ou seja,

$$
\text { vicio }_{F}(\hat{\theta})=E_{F}[s(\mathbf{y})]-t(F)=E_{F}[s(\mathbf{y})]-\theta \text {. }
$$

Efron (1979) propõe o estimador do vício aplicando o princípio de sustituição tanto na esperança como em $t(F)$, com

$$
\operatorname{vicio}_{\hat{F}}(\hat{\theta})=E_{\hat{F}}\left[s\left(\mathbf{y}^{*}\right)\right]-t(\hat{F})=E_{\hat{F}}\left[s\left(\mathbf{y}^{*}\right)\right]-\hat{\theta} .
$$

Assim o estimador bootstrap do vício $\hat{F}(\hat{\theta})$ pode ser aproximado pela simulação de Monte Carlo, isto é,

1. gerar $B$ amostras bootstrap independentes $\mathrm{y}^{* 1}, \mathrm{y}^{* 2}, \ldots, \mathrm{y}^{* B}$;

2. calcular as réplicas bootstrap $\hat{\theta}^{*}(b)=s\left(\mathbf{y}^{* b}\right)$;

3. aproximar a esperança bootstrap $E_{\hat{F}}\left[s\left(\mathbf{y}^{*}\right)\right]$ pela média, isto é,

$$
\hat{\theta}^{*}(\cdot)=\frac{1}{B} \sum_{b=1}^{B} \hat{\theta}^{*}(b)=\frac{1}{B} \sum_{b=1}^{B} s\left(\mathbf{y}^{* b}\right) ;
$$

4. finalmente obter o estimador bootstrap do vício baseado nas $B$ réplicas.

$$
\operatorname{vicio}_{B}=\hat{\theta}^{*}(\cdot)-\hat{\theta}
$$

Outra técnica bastante comum para estimação do vício e do erro padrão é o jackknife, sugerida por Quenouille (1949). Embora sendo menos eficiente, a vantagem dessa técnica sobre o bootstrap é que ela usa somente $n$ réplicas, que em geral é menor do que o número de réplicas do bootstrap. É também possível fazer o bootstrap do jackknife, podendo-se ter idéia do erro padrão do vício estimado pelo jackknife. 


\subsection{Intervalos de confiança}

Outra aplicação do bootstrap é encontrar intervalos de confiança para $\theta$. O propósito de obter o erro padrão estimado de um estimador $\hat{\theta}$ é estabelecer o intervalo de confiança $(1-2 \alpha)$ aproximado para o parâmetro desconhecido $\theta$.

O número de réplicas para a obtenção de um intervalo de confiança bootstrap é bem maior do que o usado para estimar o vício ou o erro padrão de um estimador. O número de réplicas bastante comum na literatura é 2000 .

Para introduzir a idéia do intervalo, suponha que o estimador $\hat{\theta}$ tenha uma distribuição normal com média $\theta$ e variância $\operatorname{Var}(\hat{\theta})$, isto é, $\hat{\theta} \sim N(\theta, \operatorname{Var}(\hat{\theta}))$. Assim,

$$
Z=\frac{\hat{\theta}-\theta}{\sqrt{\operatorname{Var}(\hat{\theta})}} \sim N(0,1) .
$$

O intervalo de confiança com coeficiente de confiança de $100(1-2 \alpha) \%$ é dado por

$$
\left[\hat{\theta}-z^{(1-\alpha)} \sqrt{\operatorname{Var}(\hat{\theta})} \quad ; \quad \hat{\theta}-z^{(\alpha)} \sqrt{\operatorname{Var}(\hat{\theta})}\right]
$$

onde $z^{(\alpha)}$ é o percentil de ordem $100 \alpha \%$ da distibuição normal padrão . O intervalo pode ser escrito como

$$
\hat{\theta} \pm z^{(1-\alpha)} \sqrt{\operatorname{Var}(\hat{\theta})}
$$

pois na distribuição Normal, $z^{(\alpha)}=-z^{(1-\alpha)}$.

Muitas vezes a forma da distribuição e a variância de $\hat{\theta}$ não são conhecidas e uma aproximação para esse intervalo, quando $n$ é grande, é dada por

$$
\hat{\theta} \pm z^{(1-\alpha)} \hat{e p}(\hat{\theta})
$$

onde $\hat{e p}(\hat{\theta})$ é uma estimativa do desvio padrão de $\hat{\theta}$.

O problema com esse intervalo, conhecido como intervalo padrão, é que ele é baseado em aproximações assintóticas e pode não ser muito acurado. 
Efron (1987), DiCiccio e Romano (1988), Hall (1988) e DiCiccio e Efron (1996) mostram que o bootstrap pode ser usado para produzir intervalos de confiança não paramétricos mais exatos do que o intervalo padrão.

Há vários procedimentos bootstrap para a obtenção de intervalos de confiança. Os mais comuns são o bootstrap-t e o bootstrap-p, que descrevemos nas próximas subseções.

\subsubsection{Intervalos bootstrap-t}

Sem fazer suposições sobre a distribuição de $\hat{\theta}$, este procedimento introduzido por Efron (1979) estima a distribuição de $Z$ em (2.2) diretamente a partir dos dados, isto é, a idéia é construir uma tabela bootstrap como a da distribuição normal ou a da distribuição t-Student, que seja apropriada para o conjunto de dados observados. Esta tabela é construída gerando-se $B$ amostras bootstrap, calculando-se os $z^{*}(b)$, cuja expressão é dada abaixo, e consiste dos percentis dos $B$ valores. A tabela é usada da mesma maneira como se usa as tabelas Normal e t-Student. O algoritmo do método bootstrap-t segue os seguintes passos:

1. gerar $B$ amostras bootstrap $\mathrm{y}^{* 1}, \mathrm{y}^{* 2}, \ldots, \mathrm{y}^{* B}$;

2. para cada amostra, calcular

$$
z^{*}(b)=\frac{\hat{\theta}^{*}(b)-\hat{\theta}}{\hat{e p}^{*}(b)}, \quad b=1, \ldots, B
$$

onde, $\hat{\theta}^{*}(b)=s\left(\mathrm{y}^{* b}\right)$ é o valor de $\hat{\theta}$ para a amostra $\mathrm{y}^{* b} ; \hat{e p^{*}}(b)$ é o erro padrão estimado de $\hat{\theta}^{*}$ para a amostra bootstrap $\mathrm{y}^{* b}$;

3. calcular o percentil $\alpha$ de $z^{*}(b)$ que é estimado pelo valor $\hat{t}^{(\alpha)}$, tal que,

$$
\frac{\#\left\{z^{*}(b) \leq \hat{t}^{(\alpha)}\right\}}{B}=\alpha .
$$

Para encontrar as posições onde se encontram os $\hat{t}^{(\alpha)}$ estimados, procedemos da seguinte maneira: 
$\hat{t}^{(\alpha)}$ : estará na posição $B \alpha$ da tabela dos $z^{*}(b)$ ordenados,

$\hat{t}^{(1-\alpha)}$ : estará na posição $B(1-\alpha)$ da tabela dos $z^{*}(b)$ ordenados;

4. finalmente o intervalo de confiança bootstrap-t é dado por

$$
\left(\hat{\theta}-\hat{t}^{(1-\alpha)} \hat{e p}(\hat{\theta}) \quad ; \quad \hat{\theta}-\hat{t}^{(\alpha)} \hat{e p}(\hat{\theta})\right)
$$

onde $\hat{e p}(\hat{\theta})$ é o erro padrão estimado de $\hat{\theta}$ para a amostra original.

Quando $B \alpha$ não é inteiro é possível calcular o percentil aproximado tomando em seu lugar o maior inteiro contido em $(B+1) \alpha$.

O método bootstrap-t, em geral, apresenta bons resultados quando da estimação de parâmetros de locação, embora o mesmo não aconteça quando o parâmetro de interesse é mais complicado.

O cálculo de $Z$ depende de $\hat{e p}(\hat{\theta})$ para cada amostra bootstrap, ou seja, depende de se conhecer a expressão do estimador de $e p(\hat{\theta})$, o que nem sempre acontece. Quando isto não ocorre, pode-se estimar o erro padrão através do bootstrap, mas isso aumentaria muito o número de réplicas. Se, por exemplo, usarmos 2000 réplicas para o cálculo do intervalo e para cada uma delas tomarmos 25 réplicas para a estimação do erro padrão, teremos tomado 50000 réplicas.

O que nos permite construir esse tipo de intervalo é que $Z$, dado por (2.2), tem aproximadamente a mesma distribuição, para cada valor de $\theta$.

Efron e Tibshirani (1993) mostram que, para valores grandes de $n$, a cobertura do intervalo tende a ser mais próxima do nível de confiança desejado do que no intervalo padrão ou no intervalo baseado em t-Student.

Outra observação interessante é que a tabela da distribuição Normal padrão se aplica a amostras de qualquer tamanho, a tabela da distribuição t-Student se aplica a todas as amostras de tamanho $n$ e a tabela gerada pelo bootstrap-t se aplica somente à amostra original que está sendo usada. Se outra amostra for sorteada, a tabela bootstrap-t pode ser diferente. 


\subsubsection{Intervalos bootstrap-p}

O intervalo bootstrap-p pode ser paramétrico ou não dependendo como as réplicas são sorteadas. No caso paramétrico pode-se usar o estimador de máxima verossimilhança. Os intervalos bootstrap-p são baseados na distribuição bootstrap.

Seja $\hat{G}$ a função de distribuição acumulada de $\hat{\theta}^{*}=s\left(\mathrm{Y}^{*}\right)$.

0 intervalo bootstrap-p com cobertura $1-2 \alpha$, é definido pelos percentis $\alpha$ e $1-\alpha$ de $\hat{G}$, ou seja,

$$
\left[\hat{G}^{-1}(\alpha) \quad ; \quad \hat{G}^{-1}(1-\alpha)\right] .
$$

Consideremos que $\hat{G}^{-1}(\alpha)=\hat{\theta}^{*(\alpha)}$, o $100 \alpha$-percentil empírico dos valores $\hat{\theta}^{*}(b)$. Assim, o intervalo de confiança pode ser escrito como

$$
\left[\hat{\theta}^{*(\alpha)} ; \quad \hat{\theta}^{*(1-\alpha)}\right] .
$$

Os passos do algoritmo não-paramétrico são os seguintes:

1. gerar $B$ amostras aleatórias bootstrap independentes de tamanho $n$;

2. calcular as réplicas bootstrap $\hat{\theta}^{*}(1), \hat{\theta}^{*}(2), \ldots, \hat{\theta}^{*}(B)$. Ordenar estas réplicas;

3. sejam $\hat{\theta}_{(1)}^{*}, \hat{\theta}_{(2)}^{*}, \ldots, \hat{\theta}_{(B)}^{*}$, as correspondentes estatísticas de ordem. O intervalo de confiança bootstrap-p pode ser aproximado por:

$$
\left[\hat{\theta}_{(r)}^{*} ; \quad \hat{\theta}_{(s)}^{*}\right]
$$

onde $r$ e $s$ são inteiros, com $r=B \alpha$ e $s=B(1-\alpha)$, e representam as posições na tabela dos $\hat{\theta}^{*}(b)$ ordenados.

Se $r$ e $s$ não são inteiros, é possível obter o intervalo aproximado, como no caso do intervalo bootstrap-t, tomando como base o maior inteiro contido em $(B+1) \alpha$. Johns(1988) 
mostrou como as demandas computacionais deste método percentil podem ser substancialmente reduzidas ao incorporar técnicas de importance sampling (Hammersley e Handscomb (1964)).

Um comentário interessante é que o intervalo padrão, dado no início da Seção 2.4 e o intervalo baseado na distribuição t-Student, são forçosamente simétricos, mesmo que o intervalo exato não seja. Isto não ocorre com os intervalos bootstrap-t e bootstrap-p. Se $\hat{G}$ tem cauda longa à direita, o bootstrap-t tem cauda longa à esquerda, ocorrendo o inverso com o intervalo bootstrap-p.

Se a distribuição de $\hat{\theta}^{*}$ é rudemente normal, o intervalo padrão e o bootstrap-t são próximos do bootstrap-p. Senão, o bootstrap-p, em geral, é melhor. Uma maneira de corrigir o intervalo padrão é obter o intervalo de confiança para uma transformação do parâmetro de interesse, cujo estimador tenha distribuição aproximadamente normal. Outro problema, que às vezes também pode ser corrigido por transformação, é que o erro padrão em certos casos depende de $\theta$. A transformação nesse caso tenta estabilizar a. variância. Entretanto, nem sempre uma transformação desse tipo é conhecida.

Uma desvantagem do bootstrap-p é que ele subestima as caudas da distribuição de $\hat{\theta}^{*}$.

Tanto o bootstrap-t como o bootstrap-p apresentam problemas. O bootstrap-t apresenta boa probabilidade teórica de cobertura, mas é errático. O oposto ocorre com o bootstrap-p que é menos errático mas também é menos satisfatório com relação às propriedades de cobertura.

Ambos podem ser melhorados. Duas opções de como fazê-lo são dadas em Efron e Tibshirani (1993) e DiCiccio e Efron (1996). São os métodos BCa (bias-corrected and accelerated) e ABC (approximate bootstrap confidence). O BCa melhora o bootstrap-p e o $\mathrm{ABC}$ reduz a quantidade de computação necessária para o $\mathrm{BCa}$. 


\subsection{Ilustração numérica}

Esta seção nos ajudará a visualizar a técnica bootstrap não paramétrica, mediante um exemplo, tomado do livro de Bussab e Morettin (1991).

Exemplo 2.5.1 Os dados abaixo representam a porcentagem média da receita familiar gasta com alimentação pelos moradores de uma grande vila industrial. Para isso selecionou-se uma amostra de 16 famílias que apresentou os seguintes resultados:

$\begin{array}{llllllllllllllll}41 & 44 & 35 & 42 & 34 & 22 & 42 & 42 & 38 & 62 & 29 & 63 & 38 & 45 & 48 & 40\end{array}$.

A seguir utilizaremos estes dados para estimar o erro padrão, o vício e intervalos de confiança para a média.

Consideremos os valores como observações independentes $y_{i}$ de uma distribuição contínua $F$ com média $\theta$. Os estimadores da média e de seu erro padrão e as estimativas obtidas pelas respectivas expressões são

$$
\begin{gathered}
\hat{\theta}=\bar{Y}=\frac{1}{n} \sum_{i=1}^{n} y_{i}=41,5625 \\
\hat{e p}(\hat{\theta})=\left[\frac{1}{n^{2}} \sum_{i=1}^{n}\left(y_{i}-\bar{y}\right)^{2}\right]^{1 / 2}=2,5046 .
\end{gathered}
$$

\section{Simulações bootstrap}

Tirar uma amostra bootstrap a partir de $F_{n}(y)$ é equivalente a retirar cada $y_{i}^{*}$ de uma quantidade aleatória de valores observados $y_{1}, y_{2}, \ldots, y_{16}$. Posto que $y_{i}^{*}$ são independentes, sorteamos as observações com reposição.

As simulações foram feitas primeiro para a média, erro padrão e vício e depois para os intervalos de confiança bootstrap-t e bootstrap-p.

A Tabela 2.1 abaixo mostra as estimativas da média, erro padrão e vício para o número de réplicas variando de 25 a 2000 
Tabela 2.1: Estimativas da média, erro padrão e vício

\begin{tabular}{|c|c|c|c|c|c|c|c|}
\hline B & 25 & 50 & 100 & 200 & 500 & 1000 & 2000 \\
\hline média & 42,3325 & 41,8825 & 42,0887 & 41,5094 & 41,69936 & 41,5365 & 41,4812 \\
\hline$\hat{e p}$ & 2,3778 & 2,4453 & 2,7207 & 2,6824 & 2,6151 & 2,5717 & 2,1780 \\
\hline vicio $_{B}$ & 0,7700 & 0,3200 & 0,5262 & $-0,0500$ & 0,1311 & $-0,0260$ & $-0,0813$ \\
\hline
\end{tabular}

$\mathrm{Na}$ tabela pode-se observar que:

1. quanto maior é o número de réplicas $B$ mais próximos são os valores dos estimadores bootstrap dos valores dos estimadores obtidos da amostra original;

2. os valores mais próximos dos obtidos pelas expressões ocorreram para $B=1000$. Para $\mathrm{B}=200$ que é o número de réplicas comumente usado, as estimativas bootstrap da média, erro padrão e vício são

média: $\hat{\theta}_{B}=41,5094$

erro padrão: $\hat{e p}_{B}=2,6824$

vício: $v_{i \hat{c i o}}=-0,0500$.

Os intervalos bootstrap-t e bootstrap-p foram obtidos com 2000 réplicas.

A Tabela 2.2 mostra os percentis da distribuição Normal $(0,1)$, da distribuição $t$ Student com 15 graus de liberdade e da distribuição bootstrap para o exemplo.

Tabela 2.2: Percentis das distribuições normal padrão, $t$-Student e bootstrap-t

\begin{tabular}{|c|c|c|c|c|c|c|}
\hline percentil & $1 \%$ & $5 \%$ & $10 \%$ & $90 \%$ & $95 \%$ & $99 \%$ \\
\hline Normal & $-2,33$ & $-1,64$ & $-1,28$ & 1,28 & 1.64 & 2,33 \\
\hline$t_{(n-1)}$ & -2.602 & $-1,753$ & -1.341 & 1.341 & 1.753 & 2.602 \\
\hline bootstrap- $t$ & -5.0721 & -1.8898 & -2.7609 & 3.0686 & 1.1912 & 6.3359 \\
\hline
\end{tabular}


Os intervalos de confiança. com coeficientes de confiança de $90 \%$ são dados a seguir.

Intervalo de confiança padrão

$$
\begin{aligned}
\hat{\theta} \pm z^{(1-\alpha)} \hat{e p}(\hat{\theta}) & =41,5625 \pm 1,64(2,5046) \\
I C_{(90 \%)} & =[37,4550 \quad ; \quad 45,6700]
\end{aligned}
$$

Intervalo de confiança t-Student

$$
\begin{array}{r}
\hat{\theta} \pm t_{(n-1)}^{(1-\alpha)} \hat{e p}(\hat{\theta})=41,5625 \pm 1,753(2,5046) \\
I C_{(90 \%)}=[37,1719 \quad ; \quad 45,9531]
\end{array}
$$

\section{Intervalo de confiança bootstrap-t}

Os valores utilizados correspondem às posições 100 e 1900

$$
\begin{aligned}
& \left(\hat{\theta}-\hat{t}^{(1-\alpha)} \hat{e p}(\hat{\theta}) \quad ; \quad \hat{\theta}-\hat{t}^{(\alpha)} \hat{e p}(\hat{\theta})\right) \\
& I C_{(90 \%)}=(41,5625-1,1912(2,5056) ; 41,5625-(-1,8898(2,5056))) \\
& I C_{(90 \%)}=(38,5778 ; 46,2976)
\end{aligned}
$$

\section{Intervalo de confiança bootstrap-p}

A Figura 2.1 mostra o histograma obtido através das 2000 réplicas $\hat{\theta}_{b}^{*}$ que sugere uma distribuição aproximadamente normal. Assim, tem-se que o intervalo bootstrap-p, com cobertura de $90 \%$, é dado por

$$
\begin{aligned}
& {\left[\hat{\theta}_{(r)}^{*} \quad ; \quad \hat{\theta}_{(s)}^{*}\right]=\left[\begin{array}{lll}
\hat{\theta}_{(100)}^{*} & ; & \hat{\theta}_{(1900)}^{*}
\end{array}\right]} \\
& I C_{(90 \%)}=[37,5000 ; 45,8125],
\end{aligned}
$$

onde $r$ e $s$ são as posições 100 e 1900 das estimativas $\hat{\theta}_{b}^{*}$ ordenadas. 
Figura 2.1: Histograma dos $2000 \hat{\theta}_{b}^{*}$

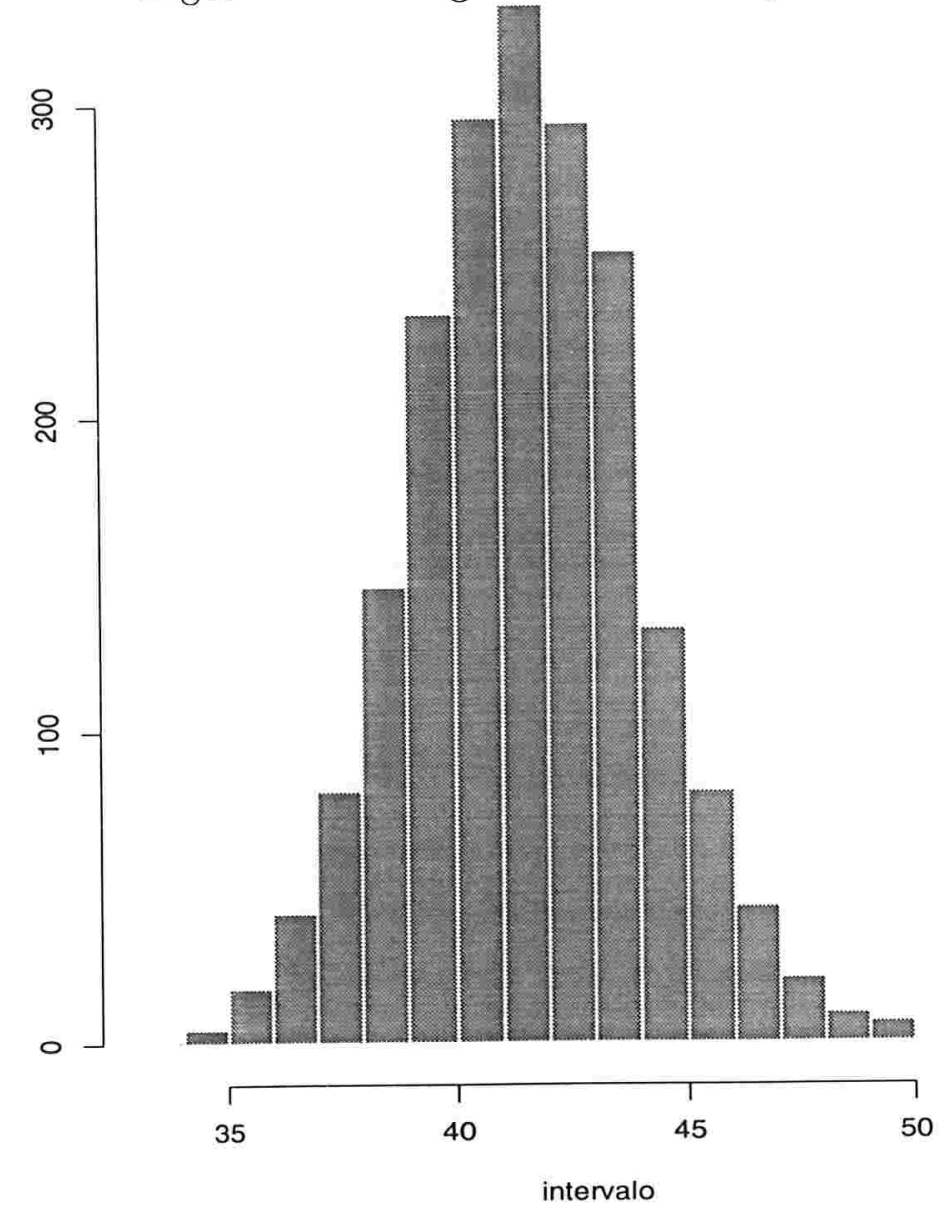

A Tabela 2.3 resume os resultados encontrados.

Tabela 2.3: Intervalos de confiança para a proporção média da receita familiar gasta com alimentação

\begin{tabular}{|c|lcl|}
\hline Intervalo de confiança & \multicolumn{3}{|c|}{ limites } \\
\hline normal & {$[37,4550 ;$} & $45,6700]$ \\
\hline t-Student & {$[37,1719 ;$} & $45,9531]$ \\
\hline bootstrap- $t$ & $(38,5778 ;$ & $46,2976)$ \\
\hline bootstrap-p & {$[37,5000 ;$} & $45,8125]$ \\
\hline
\end{tabular}


Pode-se notar que o intervalo com menor amplitude foi o obtido pelo método bootstrapt, seguido por normal, bootstrap-p e t-Student.

Os intervalos normal e t-Student são, por construção, simétricos com relação a $\hat{\theta}$. $O$ intervalo bootstrap-p resultou praticamente simétrico, o que não ocorreu com o bootstrapt, que apresentou cauda maior à direita. 


\section{Capítulo 3}

\section{Imputação de dados}

\subsection{Introdução}

Um problema bastante comum em levantamentos amostrais é a falta de algumas observações. Muitos destes problemas são de natureza multivariada. incluincio regressão, análise fatorial, análise discriminante e outros. Uma questão é como trata-los quando se tem dados ausentes em uma ou mais variáveis.

A ocorrência de dados ausentes pode ser devida à não resposta à investigação por várias razões ou à eliminação dos valores inconsistentes durante a avaliação da consistência das respostas. A não resposta pode ser de dois tipos, total ou parcial; a primeira que se caracteriza pela não resposta de uma unidade inteira da amostra selecionada e consiste na chamada não resposta da unidade, e a segunda, que se caracteriza pela não resposta a uma das variáveis investigadas, chamada não resposta de item.

Rubin (1976) classifica o mecanismo de dados ausentes de acordo com a probabilidade de não resposta. Assim, quando a probabilidade de não resposta depende dos dados presentes mas não dos ausentes, os dados são ditos perdidos por um processo aleatório (MAR- missing at random). Se o mecanismo de não resposta não depende de dados presentes ou ausentes, dizemos que os dados são perdidos por um processo totalmente 
aleatório (MCAR- missing completely at random).

Suponha que temos duas variáveis: $X$, que tem registros para todas as $n$ unidades da amostra, e $Y$, que está sujeito a não resposta. Assim, consideremos $X=\left(x_{1}, x_{2}, \ldots, x_{n}\right)$ e $Y=\left(y_{1}, y_{2}, \ldots, y_{n-m}, y_{n-m+1}, \ldots, y_{n}\right)$, onde $n-m$ das $n$ unidades em $Y$ são observadas e $m$ estão ausentes. Teremos uma das seguintes situações:

1. se a probabilidade de não resposta é independente de $X$ e $Y$, dizemos que os dados ausentes são MAR e os dados presentes são observados aleatoriamente OAR (observed at random), ou mais simplesmente que os dados faltantes são MCAR;

2. se a probabilidade de não reposta não depende de $\left(y_{n-m+1}, \ldots, y_{n}\right)$, mas depende de $\left(y_{1}, y_{2}, \ldots, y_{n-m}\right)$ ou de $X$, dizemos que os dados ausentes são MAR;

3. se a probabilidade de não resposta depende de $\left(y_{n-m+1}, \ldots, y_{n}\right)$, podendo depender de $X$ ou não, dizemos que os dados são NMAR (not missing at random).

Neste trabalho estamos supondo que os dados são MCAR, ou seja, que não há necessidade de se modelar o mecanismo de não resposta.

Little e Rubin (1987) classificam os métodos utilizados para trabalhar com dados parcialmente ausentes, agrupando-os nas seguintes categorias:

1. Procedimentos baseados em unidades observadas completamente: quando algumas variáveis não têm registro para algumas das unidades, costuma-se descartar as unidades incompletas, analisando-se somente as unidades com dados completos.

2. Procedimentos baseados em imputação: quando os valores ausentes são primeiro preenchidos e depois analisados através de métodos para dados completos. São mais indicados para a não resposta de item.

3. Procedimentos de ponderação: as inferências sobre amostras com dados completos são comumente baseadas em pesos, determinados pelo planejamento amostral. 
muitas vezes inversamente proporcionais às probabilidades de seleção. Este procedimento sugere que se modifiquem os pesos para considerar a não resposta e é geralmente aplicado quando se tem problemas de não resposta total.

4. Procedimentos baseados em modelos: neste caso supõe-se um modelo para os dados incompletos e os parâmetros são estimados com base nesse modelo.

O foco deste trabalho é a aplicação do método bootstrap a dados incompletos, caso em que o procedimento de imputação é utilizado. Método de imputação é qualquer procedimento de estimação de valores individuais ausentes em um conjunto de dados.

Na literatura há indicação de vários métodos, iterativos ou não, que podem ser usados. Esses métodos são classificados como estocásticos ou determinísticos. Algumas referências são Albieri (1989), Silva (1989), Bello (1993), Bello (1994) e Barroso (1995). Rubin (1987) sugere a imputação múltipla, que consiste em fazer a previsão por diferentes métodos e depois analisar os vários conjuntos completos.

Dentre as diversas alternativas, destacamos quatro métodos que serão utilizados neste trabalho, os quais são descritos a seguir.

\subsection{BLUP para o modelo misto}

O BLUP (Best Linear Unbiased Prediction) desenvolvido por Henderson (1950) para dados completos é um procedimento usado para estimar simultaneamente tanto as componentes fixas como as aleatórias de um modelo misto. Esse procedimento de estimação foi revisado por Robinson(1991), que apresenta uma ampla bibliografia sobre o assunto. Neste procedimento o vetor de variáveis aleatórias observadas y tem uma distribuição que depende do vetor de parâmetros fixos $\alpha$ e das realizações desconhecidas do vetor aleatório b. O modelo considerado por Henderson é

$$
\mathrm{y}=\mathrm{X} \alpha+\mathrm{Zb}+\mathrm{e}
$$


onde

y: vetor de $d$ variáveis aleatórias observáveis;

$\alpha$ : vetor de $p$ parâmetros fixos desconhecidos (efeitos fixos);

b: vetor de $q$ variáveis aleatórias não observáveis (efeitos aleatórios);

$\mathrm{X}$ e $\mathbf{Z}$ : matrizes conhecidas;

e: vetor de variáveis aleatórias (erros),

tais que

$$
\begin{aligned}
\mathbb{E}(\mathrm{b}) & =0 \\
\mathbb{E}(\mathrm{e}) & =0 \\
\mathrm{~V}=\operatorname{Var}\left(\begin{array}{l}
\mathrm{b} \\
\mathrm{e}
\end{array}\right) & =\mathrm{W} \sigma^{2}=\left(\begin{array}{ll}
\mathbf{G} & 0 \\
\mathbf{0} & \mathbf{R}
\end{array}\right) \sigma^{2},
\end{aligned}
$$

onde $\mathbf{G}$ e $\mathbf{R}$ são matrizes positivas definidas de dimensão $(q \times q)$ e $(d \times d)$, respectivamente, de posto completo e $\sigma^{2}$ é uma constante positiva, possivelmente desconhecida.

Henderson (1975) mostra que os BLUP's dos efeitos são: lineares, no sentido que eles são funções lineares dos dados y; não viesados no sentido que o valor médio do estimador é igual ao valor médio da quantidade a ser estimada e melhores, no sentido que eles têm erro quadrático médio mínimo dentro da classe dos estimadores lineares não viesados.

As estimativas BLUP's de $\alpha$ e b, segundo Henderson (1953) são definidas como soluções das equações simultâneas:

$$
\begin{array}{r}
\mathbf{X}^{T} \mathbf{R}^{-1} \mathbf{X} \hat{\alpha}+\mathbf{X}^{T} \mathbf{R}^{-1} \mathbf{Z} \hat{\mathbf{b}}=\mathbf{X}^{T} \mathbf{R}^{-1} \mathbf{y} \\
\mathbf{Z}^{T} \mathbf{R}^{-1} \mathbf{X} \hat{\boldsymbol{\alpha}}+\left(\mathbf{Z}^{T} \mathbf{R}^{-1} \mathbf{Z}+\mathbf{G}^{-1}\right) \hat{\mathbf{b}}=\mathbf{Z}^{T} \mathbf{R}^{-1} \mathbf{y}
\end{array}
$$

conhecidas como equações do modelo misto, sendo suas soluções:

$$
\begin{gathered}
\hat{\alpha}=\left(\mathrm{X}^{T} \mathbf{W}^{-1} \mathbf{X}\right)^{-1} \mathbf{X}^{T} \mathbf{W}^{-1} \mathrm{y} \\
\hat{\mathrm{b}}=\left(\mathbf{Z}^{T} \mathbf{R}^{-1} \mathrm{Z}+\mathrm{G}^{-1}\right)^{-1}\left[\mathbf{Z}^{T} \mathbf{R}^{-1}-\mathbf{Z}^{T} \mathbf{R}^{-1} \mathbf{X}\left(\mathbf{X}^{T} \mathbf{W}^{-1} \mathbf{X}\right)^{-1} \mathbf{X}^{T} \mathbf{W}^{-1}\right] \mathrm{y} .
\end{gathered}
$$


Barroso (1995) deduziu o BLUP para efeitos fixos e aleatórios na presença de dados ausentes, quando a razão entre as componentes da variância é conhecida. Estudou o caso quando $m$ das $d$ observações são ausentes e o modelo pode ser escrito da seguinte maneira:

$$
\mathrm{y}=\left(\begin{array}{c}
\mathrm{y}_{o} \\
\mathrm{y}_{a}
\end{array}\right)=\left(\begin{array}{c}
\mathrm{x}_{o} \\
\mathrm{X}_{a}
\end{array}\right) \alpha+\left(\begin{array}{c}
\mathrm{Z}_{o} \\
\mathrm{Z}_{a}
\end{array}\right) \mathrm{b}+\left(\begin{array}{c}
\mathrm{e}_{o} \\
\mathrm{e}_{a}
\end{array}\right)
$$

onde os índices $o$ e $a$ referem-se aos dados observados e ausentes, respectivamente.

Sob o modelo (3.1), com $m$ observações perdidas, o pręvisor linear não viesado de erro quadrático médio mínimo (BLUP) de $\mathrm{y}_{a}$ é dado por:

$$
\hat{\mathbf{y}}_{a}=\left(\mathbf{I}_{m}-\mathbf{E}^{T} \mathbf{Q} \mathbf{E} \mathbf{E}^{T} \mathbf{R}^{-1} \mathbf{E}\right)^{-1} \mathbf{E}^{T} \mathbf{Q F} \mathbf{F}^{T} \mathbf{R}^{-1} \mathbf{F} \mathbf{F}^{T} \mathbf{y}
$$

onde

$\mathrm{Q}=\mathbf{R}\left[\mathbf{W}^{-1} \mathbf{X}\left(\mathbf{X}^{T} \mathbf{W}^{-1} \mathbf{X}\right)^{-1} \mathbf{X}^{T} \mathbf{W}^{-1}+\mathbf{R}^{-1}-\mathbf{W}^{-1}\right] \mathbf{R} ;$

E: matriz indicadora dos dados ausentes $(d \times m)$;

F: matriz indicadora dos dados presentes $(d \times(d-m))$.

Para visualizar melhor a forma das matrizes $\mathbf{E}$ e $\mathbf{F}$, a seguir mostramos um exemplo.

Exemplo. Consideremos o caso em que três indivíduos são observados em três instantes.

Notação $y_{t i}$ : observação do $i$-ésimo indivíduo no $t$-ésimo instante

\begin{tabular}{c|ccc} 
& \multicolumn{3}{|c}{ tempo } \\
Indiv & $\mathbf{1}$ & $\mathbf{2}$ & $\mathbf{3}$ \\
\hline $\mathbf{1}$ & $y_{11}$ & $?$ & $y_{31}$ \\
$\mathbf{2}$ & $?$ & $y_{22}$ & $?$ \\
$\mathbf{3}$ & $y_{13}$ & $y_{23}$ & $?$
\end{tabular}

O sinal '?' indica a ausência da informação.

Neste caso, as matrizes $\mathbf{E}$ e $\mathbf{F}$ são: 


$$
\mathbf{E}=\left(\begin{array}{cccc}
0 & 0 & 0 & 0 \\
1 & 0 & 0 & 0 \\
0 & 0 & 0 & 0 \\
\hline 0 & 1 & 0 & 0 \\
0 & 0 & 0 & 0 \\
0 & 0 & 1 & 0 \\
0 & 0 & 0 & 0 \\
0 & 0 & 0 & 0 \\
0 & 0 & 0 & 1
\end{array}\right) \quad \mathbf{F}=\left(\begin{array}{ccccc}
1 & 0 & 0 & 0 & 0 \\
0 & 0 & 0 & 0 & 0 \\
0 & 1 & 0 & 0 & 0 \\
\hline 0 & 0 & 0 & 0 & 0 \\
0 & 0 & 1 & 0 & 0 \\
0 & 0 & 0 & 0 & 0 \\
\hline 0 & 0 & 0 & 1 & 0 \\
0 & 0 & 0 & 0 & 1 \\
0 & 0 & 0 & 0 & 0
\end{array}\right)
$$

Definindo $\hat{\mathbf{y}}=\left(\begin{array}{c}\mathbf{y}_{\circ} \\ \hat{\mathbf{y}}_{a}\end{array}\right)$, os BLUP's de $\boldsymbol{\alpha}$ e b são:

$$
\begin{gathered}
\hat{\boldsymbol{\alpha}}=\left(\mathbf{X}^{T} \mathbf{W}^{-1} \mathbf{X}\right)^{-1} \mathbf{X}^{T} \mathbf{W}^{-1} \hat{\mathbf{y}} \\
\hat{\mathbf{b}}=\left(\mathbf{Z}^{T} \mathbf{R}^{-1} \mathbf{Z}+\mathbf{G}^{-1}\right)^{-1}\left[\mathbf{Z}^{T} \mathbf{R}^{-1}-\mathbf{Z}^{T} \mathbf{R}^{-1} \mathbf{X}\left(\mathbf{X}^{T} \mathbf{W}^{-1} \mathbf{X}\right)^{-1} \mathbf{X}^{T} \mathbf{W}^{-1}\right] \hat{\mathbf{y}}
\end{gathered}
$$

Observe que as duas últimas expressões são funções de $\hat{\mathbf{y}}$, o que sugere que devemos primeiro prever $\mathbf{y}_{a}$ e depois $\boldsymbol{\alpha}$ e $\mathbf{b}$, já com os dados imputados. O caminho inverso também é possivel, isto é, prever $\boldsymbol{\alpha}$ e $\mathbf{b}$ usando os dados incompletos para depois prever $\mathbf{y}_{a}$, usando o modelo (3.1).

Em nosso caso estamos trabalhando com painéis e adotamos o modelo misto usual com dois fatores, no qual o tempo é um fator fixo e o indivíduo um fator aleatório. Esse modelo é um caso particular do modelo mais geral que acabamos de descrever. Assim.

$$
y_{t i}=\mu+\alpha_{t}+b_{i}+e_{t i} \quad t=1,2 \ldots, T \quad i=1.2, \ldots, n,
$$

onde

$y_{t i}$ : observação do indivíduo $i$ no instante $t$ 
$\mu$ : média geral;

$\alpha_{t}$ : fator fixo, medindo o efeito do tempo;

$b_{i}$ : fator aleatório, medindo o efeito do indivíduo;

$e_{t i}$ : erro associado ao indivíduo $i$ no instante $t$, sob as suposições usuais,

$$
\begin{gathered}
e_{t i} \sim N\left(0, \sigma^{2}\right) \\
b_{i} \sim N\left(0, \sigma_{b}^{2}\right) \\
e_{t i} \text { 's independentes } \\
b_{i} \text { 's independentes } \\
e_{t i} \text { 's e } b_{i} \text { 's independentes. }
\end{gathered}
$$

Definimos,

$$
\sum_{t=1}^{T} \alpha_{t}=0
$$

Seja y o vetor de todas as observações,

$$
\mathbf{y}^{T}=\left(y_{11}, y_{21}, \ldots, y_{T 1}, y_{12}, y_{22}, \ldots, y_{T 2}, \ldots, y_{1 n}, y_{2 n}, \ldots, y_{T n}\right)
$$

Uma limitação deste modelo é que ele impõe a mesma estrutura de covariância entre tempos diferentes, quer sejam próximos ou não, ou seja

$$
\mathbf{V}=\operatorname{Var}(\mathbf{y})=\mathbf{W} \sigma^{2}
$$

ou

$$
\mathbf{V}=\left[\left(\mathbf{I}_{n} \odot \mathbf{I}_{T}\right)+K\left(\mathbf{I}_{n} \odot \mathbf{J}_{T}\right)\right] \sigma^{2}
$$

onde

$\mathrm{I}_{n}$ : matriz identidade de dimensão $n$;

$\mathbf{J}_{T}$ : matriz quadrada de 1's de dimensão $T$; 
$\otimes$ : produto de Kronecker;

$K=\sigma_{b}^{2} / \sigma^{2}:$ razão entre as componentes da variância.

Para estimar as componentes da variância, utilizamos os estimadores usuais obtidos através da tabela da ANOVA, truncado no zero, no caso da variância do efeito aleatório.

\subsubsection{O método de imputação}

Consideramos o caso em que $m$ observações são perdidas em um dos instantes. Consideramos que os primeiros $n-m$ indivíduos têm os dados completos e que os últimos $m$ tiveram observações ausentes no instante $\mathrm{T}$.

O BLUP para as observações ausentes é dado por

$$
\hat{y}_{T i}=\bar{y}_{T .}+\left(\frac{K(T-1)}{K(T-1)+1}\right)\left(\bar{y}_{. i}-\bar{y}_{c}\right)
$$

para $i=n-m+1, \ldots, n$, onde

$\bar{y}_{c}=\frac{1}{(n-m)(T-1)} \sum_{t=1}^{T-1} \sum_{i=1}^{n-m} y_{t i}$

$\bar{y}_{c}$ : média da parte completa, isto é, média das observações dos $n-m$ indivíduos com dados completos, nos $T-1$ primeiros instantes;

$\bar{y}_{T .}=\frac{1}{n-m} \sum_{i=1}^{n-m} y_{T i}$

$\bar{y}_{T}$ : média das observações presentes no instante $T$;

$\bar{y}_{. i}=\frac{1}{T-1} \sum_{t=1}^{T-1} y_{t i}$

$\bar{y}_{. i}$ : média das observações do $i$-ésimo indivíduo (incompleto), nos $T-1$ primeiros instantes.

No caso, $K$ é estimado com base em um conjunto de dados completos, que pode ser o conjunto de $n-m$ dados completos em $T$ instantes ou o conjunto de $n$ observações nos $T-1$ primeiros instantes. Não há conclusòes sobre qual é o melhor conjunto completo a ser usado, por isso decidimos pelo maior deles. O estimador usado é $\hat{K}=\hat{\sigma}_{b}^{2} / \hat{\sigma}^{2}$, que depende dos estimadores das componentes de variância, dados a seguir. 


\subsubsection{Método de estimação das componentes da variância}

Quando não se conhecem as componentes da variância é comum substituí-las por valores estimados obtidos por estimadores consistentes. Para o modelo adotado, o estimador MINQUE (Minimum Norm Quadratic Unbiased Estimation) das componentes de variância, sob norma Euclideana. é o mesmo estimador da ANOVA truncado no zero, que por sua vez é o mesmo que o estimador de máxima verossimilhança restrito, o REML (Restricted Estimator Maximum Likelihood) sob normalidade de efeitos e erros.

Assim as componentes da variância foram estimadas pelo estimador ANOVA, truncado no zero, dado por

$$
\begin{gathered}
\hat{\sigma}^{2}=\frac{A-B}{\left(n_{c}-1\right)\left(T_{c}-1\right)} \\
\hat{\sigma}_{b}^{2}=\left\{\begin{array}{ll}
s=\frac{T_{c} B-A}{T_{c}\left(n_{c}-1\right)\left(T_{c}-1\right)} & \text { se } s \geq 0 \\
0 & \text { se } s<0
\end{array},\right.
\end{gathered}
$$

onde $n_{c}$ e $T_{c}$ são respectivamente o número de indivíduos e o número de instantes do conjunto de dados utilizado para o cálculo das estimativas e

$$
\begin{aligned}
& A=\sum_{i=1}^{n_{c}} \sum_{t=1}^{T_{c}}\left(y_{t i}-\bar{y}_{t .}\right)^{2} \\
& B=\sum_{i=1}^{n_{c}} \sum_{t=1}^{T_{c}}\left(\bar{y}_{. i}-\bar{y}_{. .}\right)^{2} .
\end{aligned}
$$

As variâncias dos estimadores $\hat{\sigma}^{2}$ e $\hat{\sigma}_{b}^{2}$, cujas deduções são apresentadas em Searle (1971), são dadas por

$$
\begin{gathered}
\operatorname{Var}\left(\hat{\sigma}^{2}\right)=\frac{2}{\left(n_{c}-1\right)\left(T_{c}-1\right)} \sigma^{4} \\
\operatorname{Var}(s)=\frac{2}{\left(n_{c}-1\right)} \sigma_{b}^{4}+\frac{2}{T_{c}\left(n_{c}-1\right)\left(T_{c}-1\right)} \sigma^{4}+\frac{4}{T_{c}\left(n_{c}-1\right)} \sigma_{b}^{2} \sigma^{2}
\end{gathered}
$$

onde

$$
\mathbb{E}\left(e_{t i}^{2}\right)=\sigma^{2}, \quad \mathbb{E}\left(\epsilon_{t i}^{4}\right)=3 \sigma^{4}, \quad \mathbb{E}\left(b_{t i}^{2}\right)=\sigma_{b}^{2} \quad \text { e } \quad \mathbb{E}\left(b_{t i}^{4}\right)=3 \sigma_{b}^{4} .
$$


Três exemplos são dados a seguir, com o objetivo de ilustrar a aplicação deste método de imputação.

Exemplo 3.2.1 Consideremos o caso onde em um instante de tempo, observou-se uma variável para 10 unidades amostrais e três delas são perdidas.

$$
\mathrm{y}^{T}=\left(\begin{array}{lllllll}
14,23 & 12,18 & 19,21 & 16,38 & 20,63 & 15,51 & 17,43
\end{array} \quad ? \quad ? \quad ?\right)
$$

Tem-se que $n=10, T=1$ e $m=3$. Neste caso, o modelo se reduz a

$$
y_{i}=\mu+b_{i}
$$

e o BLUP a $\hat{y}_{i}=\bar{y}=\frac{1}{n-m} \sum_{i=1}^{n-m} y_{i}$. Assim, $\bar{y}=16,51$ e o vetor de dados, com os valores já imputados é

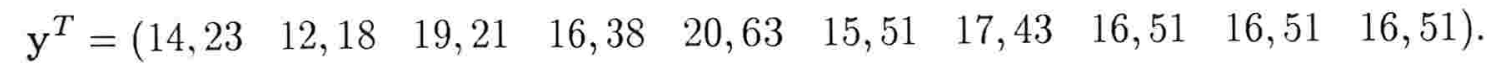

Exemplo 3.2.2 Consideremos o caso onde 7 indivíduos são observados em dois instantes de tempo e para três deles a última observação é perdida.

$$
\mathbf{Y}=\left(\begin{array}{cc}
32,81 & 29,93 \\
41,39 & 42,12 \\
28,61 & 35,02 \\
40,73 & 39,11 \\
36,08 & ? \\
30,25 & ? \\
37,14 & ?
\end{array}\right) .
$$

Temos que, $n=7, T=2$ e $m=3$. Para estimar as componentes da variância foram utilizadas as informações dos 4 primeiros indivíduos nos 2 instantes. Os resultados foram

$$
\begin{aligned}
& A=\sum_{i=1}^{4} \sum_{t=1}^{2}\left(y_{t i}-\bar{y}_{t .}\right)^{2}=199,9040 \\
& B=\sum_{i=1}^{4} \sum_{t=1}^{2}\left(\bar{y}_{. i}-\bar{y}_{. .}\right)^{2}=174,5053 \\
& \hat{\sigma}^{2}=\frac{A-B}{(4-1)(2-1)}=8,4662
\end{aligned}
$$


$\hat{\sigma}_{b}^{2}=\frac{2 B-A}{2(4-1)(2-1)}=24,8511$

$\hat{K}=\hat{\sigma}_{b}^{2} / \hat{\sigma}^{2}=2,9353$

$\bar{y}_{c}=\frac{1}{(n-m)(T-1)} \sum_{t=1}^{T-1} \sum_{i=1}^{n-m} y_{t i}=\frac{1}{4}(143.54)=35,885$

$\bar{y}_{T .}=\frac{1}{n-m} \sum_{i=1}^{n-m} y_{T i}=\frac{1}{4}(146,18)=36,545$

$\bar{y}_{.5}=36,08$

$\bar{y}_{.6}=30,25$

$\bar{y}_{.7}=37,14$.

Assim,

$$
\begin{aligned}
& \hat{y}_{25}=36,545+\left(\frac{2,9353}{2,9353+1}\right)(36,08-35,885)=36,6904 \\
& \hat{y}_{26}=36,545+\left(\frac{2,9353}{2,9353+1}\right)(30,25-35,885)=32,3419 \\
& \hat{y}_{2 \tau}=36,545+\left(\frac{2,9353}{2,9353+1}\right)(37,14-35,885)=37,4811
\end{aligned}
$$

e escrevendo a matriz com os dados já imputados, temos

$$
\mathbf{Y}=\left(\begin{array}{cc}
32,81 & 29,93 \\
41,39 & 42,12 \\
28,61 & 35,02 \\
40,73 & 39,11 \\
36,08 & 36,69 \\
30,25 & 32,34 \\
37,14 & 37,48
\end{array}\right)
$$

Exemplo 3.2.3 Consideremos o caso onde 10 indivíduos são observados em 4 instantes de tempo e no último instante as 4 últimas observações são perdidas. Então $n=10, T=4$ 
e $m=4$

$$
\mathbf{Y}=\left(\begin{array}{cccc}
6,669 & 4,376 & 4,994 & 3,539 \\
4,937 & 4,676 & 2,996 & 1,821 \\
5,285 & 4,252 & 2,697 & 0,775 \\
1,977 & 2,388 & 2,278 & 0,904 \\
3,885 & 2,594 & 0,441 & 1,035 \\
3,639 & 3,614 & 3,987 & 2,872 \\
6,035 & 4,441 & 3,839 & ? \\
5,519 & 4,552 & 3,254 & ? \\
3,890 & 4,849 & 4,684 & ? \\
6,309 & 5,429 & 5,742 & ?
\end{array}\right)
$$

Neste caso, para estimar $K$ foram usados os dados dos 10 indivíduos nos 3 primeiros instantes, o que resultou em

$$
\begin{aligned}
& A=\sum_{i=1}^{10} \sum_{t=1}^{3}\left(y_{t i}-\bar{y}_{t .}\right)^{2}=48,3799 \\
& B=\sum_{i=1}^{10} \sum_{t=1}^{3}\left(\bar{y}_{. i}-\bar{y}_{. .}\right)^{2}=36,4012 \\
& \hat{\sigma}^{2}=\frac{A-B}{(10-1)(3-1)}=0,6655 \\
& \hat{\sigma}_{b}^{2}=\frac{3 B-A}{3(10-1)(3-1)}=1,1264 \\
& \hat{K}=\hat{\sigma}_{b}^{2} / \hat{\sigma}^{2}=1,6926 \\
& \bar{y}_{c}=3,6492 \quad \bar{y}_{4 .}=1,8243 \\
& \bar{y}_{.7}=4,7717 \quad \bar{y}_{.8}=4,4417 \quad \bar{y}_{.9}=4,4743 \quad \bar{y}_{.10}=5,8267
\end{aligned}
$$

Assim, os BLUP's são

$$
\begin{aligned}
& \hat{y}_{47}=1,8243+\left(\frac{1,6926(3)}{1,6926(3)+1}\right)(4,7717-3,6492)=2,7621 \\
& \hat{y}_{48}=1,8243+\left(\frac{1,6926(3)}{1,6926(3)+1}\right)(4,4417-3,6492)=2,4864 \\
& \hat{y}_{49}=1,8243+\left(\frac{1,6926(3)}{1,6926(3)+1}\right)(4,4743-3,6492)=2,5136 \\
& \hat{y}_{410}=1,8243+\left(\frac{1,6926(3)}{1,6926(3)+1}\right)(5,8267-3,6492)=3,6435
\end{aligned}
$$


e o conjunto com dados imputados

$$
\mathbf{Y}=\left(\begin{array}{llll}
6,669 & 4,376 & 4,994 & 3,539 \\
4,937 & 4,676 & 2,996 & 1,821 \\
5,285 & 4,252 & 2,697 & 0,775 \\
1,977 & 2,388 & 2,278 & 0,904 \\
3,885 & 2,594 & 0,441 & 1,035 \\
3,639 & 3,614 & 3,987 & 2,872 \\
6,035 & 4,441 & 3,839 & 2,762 \\
5,519 & 4,552 & 3,254 & 2,486 \\
3,890 & 4,849 & 4,684 & 2,514 \\
6,309 & 5,429 & 5,742 & 3,643
\end{array}\right) .
$$

\subsection{Método de Substituição da Média (MSM)}

Este método, sugerido por Wilks (1932), é talvez o mais antigo e o mais usado, devido à sua facilidade de aplicação.

A idéia intuitiva deste método é substituir os valores faltantes de uma determinada variável pela média dos dados disponíveis. No caso de painel, onde as observações são repetidas no tempo, essa média pode ser a média do indivíduo com dado ausente ou então a média das observações presentes no instante em que a imputação está sendo feita. Cabe salientar que este método ignora, no caso multivariado, a correlação que frequentemente existe entre variáveis medidas em um mesmo indivíduo e também a intercorrelação quando as medidas são feitas no tempo.

Além disso, como vários dados ausentes são substituídos por um mesmo valor, a média, esse método cria picos na distribuição empírica, fazendo com que variâncias e covariâncias sejam subestimadas.

Como solução desse problema, a literatura (ver por exemplo Bello, 1993) dá duas sugestões: 
1. Ajustar os graus de liberdade no cálculo das estatísticas: denota-se por $s_{t t^{\prime}}$ o $\left(t . t^{\prime}\right)$-ésimo elemento da matriz de covariância ajustada $\mathbf{S}$, definida como

$$
s_{t t^{\prime}}=\frac{\sum_{i=1}^{n}\left(y_{t i}-\bar{y}_{t .}\right)\left(y_{t^{\prime} i}-\bar{y}_{t^{\prime} .}\right)}{n-c_{t t^{\prime}}-1} \quad t, t^{\prime}=1, \ldots, T
$$

onde

$$
c_{t t^{\prime}}=\left\{\begin{array}{ll}
\text { número de valores imputados no instante } t, & \text { se } s_{t t^{\prime}}=s_{t t} \\
\text { número de valores com } y_{t i}, y_{t^{\prime} i} \text { ou ambos imputados, } & \text { caso contrário }
\end{array} .\right.
$$

2. Adicionar uma pequena perturbação a cada valor imputado: consiste em substituir o valor ausente $y_{t i}$ pela média mais uma variável aleatória $\varepsilon_{i}$ com média zero e variância igual à variância da variável que está sofrendo imputação. A perturbação $\varepsilon_{i}$, pode por exemplo ser sorteada de um conjunto de dados gerados com distribuição normal ou então ser sorteada de um banco de resíduos construído das observações presentes. A imputação simples da média é a versão determinística do método, enquanto que a imputação da média mais uma perturbação é sua versão estocástica. Essa última é útil na imputação múltipla (Rubin (1978)) uma vez que pode-se substituir cada valor perdido por dois ou mais valores para representar a distribuição de probabilidades. Outros métodos de imputação determinísticos também têm sua versão estocástica, analogamente a este.

Os mesmos exemplos da seção anterior foram utilizados para ilustrar a aplicação deste método e são dados a seguir.

Exemplo 3.3.1 Consideremos os dados do exemplo 3.2.1. Neste caso, temos que $n=10, m=3, T=1$. Substituímos os valores faltantes pela média dos dados disponíveis, ou seja,

$$
\bar{y}=\frac{1}{n-m} \sum_{i=1}^{n-m} y_{i}=\frac{1}{7}(115,57)=16,51
$$


Assim. o vetor de observações completo é

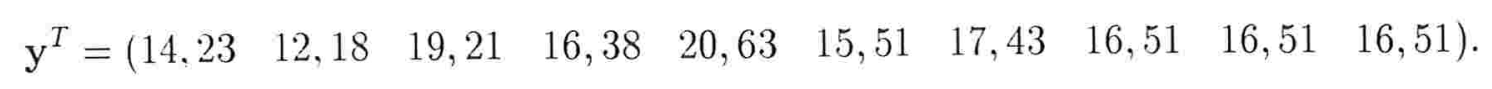

Posto que os dados ausentes foram substituídos pelo mesmo valor, este fato faz com que as variâncias e covariâncias sejam subestimadas. O cálculo da variância ajustada pelo número de graus de liberdade é

$$
S^{2}=\frac{\sum_{i=1}^{10}\left(y_{1 i}-\bar{y}_{1}\right)^{2}}{n-c-1}=\frac{50,075}{10-3-1}=8,3458
$$

Note que, para o tipo de informação considerada neste exemplo, os critérios MSM e BLUP resultam nos mesmos valores imputados. Outra observação interessante é que se a variância fosse estimada somente com a parte completa das observações, o valor da estimativa seria o mesmo, isto é, 8,3458 enquanto que se fosse estimada sem o ajuste do número de graus de liberdade seria 5,5639. Isso dá idéia de quanto o critério sem ajuste afeta a estimativa da variância.

Exemplo 3.3.2 Consideremos os dados do exemplo 3.2.2. Neste caso pode-se optar pela média das observações no segundo instante, ou pela média de cada indivíduo. No primeiro caso, os valores imputados seriam todos iguais, ou seja,

$$
\bar{y}_{T .}=\frac{1}{n-m} \sum_{i=1}^{n-m} y_{T i}=\frac{1}{4}(146,18)=36,545 .
$$

Escrevendo a matriz com os dados já imputados temos

$$
\mathbf{Y}=\left(\begin{array}{cc}
32,81 & 29,93 \\
41,39 & 42,12 \\
28,61 & 35,02 \\
40,73 & 39,11 \\
36,08 & 36,54 \\
30,25 & 36,54 \\
37,14 & 36,54
\end{array}\right)
$$


No segundo caso, temos

$$
\mathbf{Y}=\left(\begin{array}{cc}
32,81 & 29,93 \\
41,39 & 42,12 \\
28,61 & 35,02 \\
40,73 & 39,11 \\
36,08 & 36,08 \\
30,25 & 30,25 \\
37,14 & 37,14
\end{array}\right)
$$

Exemplo 3.3.3 Consideremos os dados do exemplo 3.2.3. Como no exemplo anterior é possível obter os dois tipos de médias.

1. Média do indivíduo.

$$
\begin{aligned}
& \bar{y}_{. i}=\frac{1}{T-1} \sum_{t=1}^{T-1} y_{t i}, \quad \text { para } i=n-m+1, \ldots, n \\
& \bar{y}_{.7}=\frac{1}{3} \sum_{t=1}^{3} y_{t 7}=\frac{1}{3}(14,315)=4,7717 \\
& \bar{y}_{.8}=\frac{1}{3} \sum_{t=1}^{3} y_{t 8}=\frac{1}{3}(13,325)=4,4417 \\
& \bar{y}_{.9}=\frac{1}{3} \sum_{t=1}^{3} y_{t 9}=\frac{1}{3}(13,423)=4,4743 \\
& \bar{y}_{.10}=\frac{1}{3} \sum_{t=1}^{3} y_{t 10}=\frac{1}{3}(17,480)=5,8267 .
\end{aligned}
$$

Assim, a matriz com os dados imputados é:

$$
\mathbf{Y}=\left(\begin{array}{llll}
6,669 & 4,376 & 4,994 & 3,539 \\
4,937 & 4,676 & 2,996 & 1,821 \\
5,285 & 4,252 & 2,697 & 0,775 \\
1,977 & 2,388 & 2,278 & 0,904 \\
3,885 & 2,594 & 0,441 & 1,035 \\
3,639 & 3,614 & 3,987 & 2,872 \\
6,035 & 4,441 & 3,839 & 4,772 \\
5,519 & 4,552 & 3,254 & 4,442 \\
3,890 & 4,849 & 4,684 & 4,474 \\
6,309 & 5,429 & 5,742 & 5,827
\end{array}\right) .
$$


2. Média das observações no instante $T=4$

$$
\bar{y}_{T .}=\frac{1}{n-m} \sum_{i=1}^{n-m} y_{T i}=\frac{1}{6}(10,946)=1,8243 .
$$

Então a matriz com os dados imputados estará dada por

$$
\mathbf{Y}=\left(\begin{array}{cccc}
6,669 & 4,376 & 4,994 & 3,539 \\
4,937 & 4,676 & 2,996 & 1,821 \\
5,285 & 4,252 & 2,697 & 0,775 \\
1,977 & 2,388 & 2,278 & 0,904 \\
3,885 & 2,594 & 0,441 & 1,035 \\
3,639 & 3,614 & 3,987 & 2,872 \\
6,035 & 4,441 & 3,839 & 1,824 \\
5,519 & 4,552 & 3,254 & 1,824 \\
3,890 & 4,849 & 4,684 & 1,824 \\
6,309 & 5,429 & 5,742 & 1,824
\end{array}\right)
$$

\subsection{Método de Componentes Principais de Dear (DPC)}

A análise de componentes principais é uma das técnicas multivariadas mais usadas, posto que não requer suposições sobre a distribuição dos dados. Dear (1959) usou a primeira componente principal para fazer imputação, da seguinte maneira:

1. definir uma matriz indicadora $(n \times T)$ de valores faltantes $\mathbf{H}=\left\{h_{t i}\right\}$, onde

$$
h_{t i}=\left\{\begin{array}{ll}
0 & \text { se } y_{t i} \text { é ausente } \\
1 & \text { se } y_{t i} \text { é observado }
\end{array} ;\right.
$$

2. transformar as variáveis $Y$ em $Z$, mediante a padronização

$$
\tilde{z}_{t i}=\frac{y_{t i}-\bar{y}_{t .}}{\sqrt{s_{t t}}} \quad t=1,2, \ldots, T
$$


onde $\bar{y}_{t}$. e $s_{t t}$ são a média e a variância dos dados disponíveis da $t$-ésima variável. respectivamente. Obter a matriz de correlação R, também com os dados disponíveis. ou seja, para o cálculo da correlação $r_{t t^{\prime}}$, usar as observações simultaneamente presentes em $t$ e $t^{\prime}$

3. calcular o maior autovalor da matriz de correlação $\mathbf{R}, \lambda_{1}=\max _{t}\left(\lambda_{t}\right)$ e o autovetor associado a ele, $\boldsymbol{\eta}_{1}$. Seja $\eta_{1 t}$ a $t$-ésima componente desse vetor;

4. definir o valor da primeira componente principal para o $i$-ésimo caso, como

$$
\gamma_{i}=\sum_{t=1}^{T} \eta_{1 t} z_{t i} h_{t i}
$$

isto é, o valor é dado pela combinação dos dados presentes de cada caso;

5. reconstruir os dados padronizados por

$$
\hat{z}_{t i}=\left\{\begin{array}{ll}
z_{t i} & \text { se } h_{t i}=1 \\
\eta_{1 t} \gamma_{i} & \text { se } h_{t i}=0
\end{array} ;\right.
$$

6. fazer a transformação inversa da padronização para obter os dados originais com a imputação.

Neste caso, a ilustração foi feita somente para o terceiro exemplo. 
Exemplo 3.4.1 Consideremos os dados do exemplo 3.2.3. Para aplicar o método de componentes principais de Dear, primeiro definimos a matriz indicadora $H=\left\{h_{t i}\right\}$,

$$
\mathbf{H}=\left(\begin{array}{llll}
1 & 1 & 1 & 1 \\
1 & 1 & 1 & 1 \\
1 & 1 & 1 & 1 \\
1 & 1 & 1 & 1 \\
1 & 1 & 1 & 1 \\
1 & 1 & 1 & 1 \\
1 & 1 & 1 & 0 \\
1 & 1 & 1 & 0 \\
1 & 1 & 1 & 0 \\
1 & 1 & 1 & 0
\end{array}\right) .
$$

A matriz dos dados padronizados é

$$
\mathbf{Z}=\left(\begin{array}{cccc}
1,2748 & 0,2662 & 0,9880 & 1,4914 \\
0,0842 & 0,5747 & -0,3256 & -0,0029 \\
0,3234 & 0,1387 & -0,5222 & -0,9127 \\
-1,9505 & -1,7781 & -0,7976 & -0,8005 \\
-0,6389 & -1,5662 & -2,0054 & -0,6866 \\
-0,8080 & -0,5174 & 0,3260 & 0,9113 \\
0,8390 & 0,3331 & 0,2287 & ? \\
0,4843 & 0,4472 & -0,1560 & ? \\
-0,6355 & 0,7526 & 0,7842 & ? \\
1,0273 & 1,3491 & 1,4798 & ?
\end{array}\right)
$$

O maior autovalor da matiz de correlação é $\lambda_{1}=3,0869$ e o autovetor associado a ele, $\eta_{1}^{T}=(-0.539-0,489-0,506-0,463)$. Assim, a primeira componente principal para o $i$-ésimo indivíduo tem a forma

$$
\gamma_{i}=\eta_{11} z_{1 i} h_{1 i}+\eta_{12} z_{2 i} h_{2 i}+\eta_{13} z_{3 i} h_{3 i}+\eta_{14} z_{4 i} h_{4 i}
$$


Os valores das componentes principais para os dados faltantes são

$$
\begin{aligned}
& \gamma_{7}=-0,539(0,8390)-0,489(0,3331)-0,506(0,2287)-0,463(0)=-0,7308 \\
& \gamma_{8}=-0,539(0,4843)-0,489(0,4472)-0,506(-0,1560)-0,463(0)=-0,4008 \\
& \gamma_{9}=-0,539(-0,6355)-0,489(0,7526)-0.506(0,7842)-0,463(0)=-0,4223 \\
& \gamma_{10}=-0,539(1,0273)-0,489(1,3491)-0,506(1,4798)-0,463(0)=-1,9622 .
\end{aligned}
$$

Assim, os valores imputados da matriz padronizada são

$$
\begin{aligned}
& \hat{z}_{47}=\eta_{14} \gamma_{7}=-0,463(-0,7308)=0,3384 \\
& \hat{z}_{48}=\eta_{14} \gamma_{8}=-0,463(-0,4008)=0,1856 \\
& \hat{z}_{49}=\eta_{14} \gamma_{9}=-0,463(-0,4223)=0,1955 \\
& \hat{z}_{410}=\eta_{14} \gamma_{10}=-0,463(-1,9622)=0,9085
\end{aligned}
$$

e, para voltarmos à variável original, consideramos $\sqrt{s_{44}}=1,1497, \bar{y}_{4 .}=1,8243$ e temos

$$
\begin{aligned}
& \hat{y}_{47}=0,3384(1,1497)+1,8243=2,2134 \\
& \hat{y}_{48}=0,1856(1,1497)+1,8243=2,0377 \\
& \hat{y}_{49}=0,1955(1,1497)+1,8243=2,0491 \\
& \hat{y}_{410}=0,9085(1,1497)+1,8243=2,8688 .
\end{aligned}
$$

A matriz com os dados já imputados é dada por

$$
\mathbf{Y}=\left(\begin{array}{cccc}
6,669 & 4,376 & 4,994 & 3,539 \\
4,937 & 4,676 & 2,996 & 1,821 \\
5,285 & 4,252 & 2,697 & 0,775 \\
1,977 & 2,388 & 2,278 & 0,904 \\
3,885 & 2,594 & 0,441 & 1,035 \\
3,639 & 3,614 & 3,987 & 2,872 \\
6,035 & 4,441 & 3,839 & 2,213 \\
5,519 & 4,552 & 3,254 & 2,038 \\
3,890 & 4,849 & 4,684 & 2,049 \\
6,309 & 5,429 & 5,742 & 2,869
\end{array}\right)
$$




\subsection{O algoritmo EM}

Uma estratégia computacional para problemas com dados ausentes é o Algoritmo Expectation-Maximization (EM). Dempster, Laird e Rubin (1977) introduziram este algoritmo para calcular os estimadores de máxima verossimilhança a partir de dados ausentes. A idéia principal do algoritmo EM tem sido apresentada por muitos autores para casos especiais como o da família exponencial. A desvantagem deste algoritmo é que sua taxa de convergência, quando existem muitos dados ausentes, é baixa.

O artigo de Dempster, Laird e Rubin tem dado três contribuições significativas sobre o assunto:

1. reconhecer os dois passos do algoritmo EM, cálculo da esperança (passo-E) e maximizacão (passo-M) em suas formas gerais;

2. dar algumas propriedades teóricas do algoritmo;

3. reconhecer e dar uma ampla variedade de aplicações em estatística.

O algoritmo EM formaliza uma idéia relativamente antiga de imputação de valores ausentes e iteração, isto é,

1. substituir valores ausentes por valores previstos;

2. estimar parâmetros;

3. prever novamente os valores ausentes assumindo que os novos estimadores dos parâmetros são corretos;

4. re-estimar parâmetros e assim sucessivamente, até a convergência.

No passo-M do algoritmo, executa-se a estimação de máxima verossimilhança do parâmetro considerando os dados "completados", enquanto que no passo-E, encontrase a esperança condicional dos dados ausentes, conhecendo-se os dados presentes e os atuais parâmetros estimados. Os dados ausentes são substituidos por essas esperanças. 


\subsubsection{Descrição do algoritmo}

Para descrever com mais detalhe este algoritmo, assumimos que tem-se a seguinte situação. Sejam $Y_{1}, Y_{2}, \ldots, Y_{T}, T$ variáveis aleatórias independentes com uma distribuição normal $T$-variada, com média $\boldsymbol{\mu}=\left(\mu_{1}, \ldots, \mu_{T}\right)$ e matriz de covariância $\boldsymbol{\Sigma}=\left(\sigma_{t t^{\prime}}\right)$. Consideremos $\mathrm{y}_{\mathbf{i}}^{T}=\left(\mathrm{y}_{o i}^{T}, \mathrm{y}_{a i}^{T}\right)$ a $i$-ésima observação de uma amostra de tamanho $n$, $\mathbf{y}_{i}^{T}=\left(y_{1 i}, y_{2 i}, \ldots, y_{T i}\right)$, rearranjada e particionada de modo que $\mathrm{y}_{o i}$ indica o conjunto de dados observados e $\mathrm{y}_{a i}$ o conjunto de dados ausentes do $i$-ésimo indivíduo.

Consideremos a hipótese de que os dados completos y pertencem à família exponencial regular com estatísticas suficientes,

$$
S= \begin{cases}\sum_{i=1}^{n} y_{t i} & t=1, \ldots, T \\ \sum_{i=1}^{n} y_{t i} y_{t^{\prime} i} & t, t^{\prime}=1, \ldots, T\end{cases}
$$

e derivemos o algoritmo EM na k-ésima iteração.

Seja $\hat{\boldsymbol{\theta}}^{(k)}=\left(\hat{\boldsymbol{\mu}}^{(k)}, \hat{\boldsymbol{\Sigma}}^{(k)}\right)$ o estimador de máxima verossimilhança do parâmetro $\theta=(\boldsymbol{\mu}, \boldsymbol{\Sigma})$ na $k$-ésima iteração. Os passos $\mathrm{E}$ e $\mathrm{M}$ do algoritmo são ilustrados como segue:

Passo E: consiste em calcular,

$$
\begin{array}{r}
\mathbb{E}\left(\sum_{i=1}^{n} y_{t i} / \mathrm{y}_{o i}, \hat{\boldsymbol{\theta}}^{(k)}\right)=\sum_{i=1}^{n} y_{t i}^{(k)}, \quad t=1, \ldots, T \\
\mathbb{E}\left(\sum_{i=1}^{n} y_{t i} y_{t^{\prime} i} / \mathrm{y}_{o i}, \hat{\boldsymbol{\theta}}^{(k)}\right)=\sum_{i=1}^{n}\left(y_{t i}^{(k)} y_{t^{\prime} i}^{(k)}+c_{t t^{\prime} i}^{(k)}\right), \quad t, t^{\prime}=1, \ldots, T
\end{array}
$$

onde

$$
y_{t i}^{(k)}= \begin{cases}y_{t i}, & \text { se } y_{t i} \text { é observado } \\ \mathbb{E}\left(y_{t i} / \mathrm{y}_{o i}, \hat{\theta}^{(k)}\right), & \text { se } y_{t i} \text { é ausente }\end{cases}
$$

e

$$
c_{t t^{\prime} i}^{(k)}=\left\{\begin{array}{ll}
0, & \text { se } y_{t i} \text { ou } y_{t^{\prime} i} \text { são observados } \\
\operatorname{Cov}\left(y_{t i}, y_{t^{\prime} i} / \mathbf{y}_{o i}, \hat{\theta}^{(k)}\right), & \text { se } y_{t i} \text { e } y_{t^{\prime} i} \text { são ausentes }
\end{array} .\right.
$$


Os valores ausentes $\mathrm{y}_{o i}$ são substituídos pela média condicional de $\mathrm{y}_{a i}$ dado o conjunto de valores $\mathrm{y}_{o i}$ observados para a unidade $i$.

Passo M: os novos estimadores $\hat{\theta}^{(k+1)}=\left(\hat{\boldsymbol{\mu}}^{(k+1)}, \hat{\Sigma}^{(k+1)}\right)$ são estimados a partir das estatísticas suficientes dos dados completos, isto é;

$$
\mu_{t}^{(k+1)}=\frac{1}{n} \sum_{i=1}^{1} y_{t i}^{(k)}, \quad t=1, \ldots, T
$$

e

$$
\sigma_{t t^{\prime}}^{(k+1)}=\frac{1}{n} \sum_{i=i}^{n}\left[\left(y_{t i}^{(k)}-\mu_{t}^{(k+1)}\right)\left(y_{t^{\prime} i}^{(k)}-\mu_{t^{\prime}}^{(k+1)}\right)+c_{t t^{\prime} i}^{(k)}\right], \quad t, t^{\prime}=1, \ldots, T .
$$

O algoritmo prossegue de maneira iterativa até que a diferença entre $\hat{\theta}^{(k)}$ e $\hat{\theta}^{(k+1)}$ seja pequena. No final, os valores ausentes são substituídos pelos valores obtidos na última realização do passo E.

Neste caso, também foi usado o terceiro exemplo como ilustração.

Exemplo 3.5.1 Consideremos os dados do exemplo 3.2.3.

Para aplicar o algoritmo EM, fizemos os seguintes passos

1. como critério de parada usamos a diferença entre os logaritmos das funções de verossimilhanças em duas etapas consecutivas. Para que o processo fosse interrompido, definimos que essa diferença fosse menor do que 0,001. A densidade da normal multivariada é dada por

$$
f(\mathrm{y})=\frac{1}{|2 \pi \boldsymbol{\Sigma}|^{2}} \exp \left\{-\frac{1}{2}(\mathrm{y}-\boldsymbol{\mu})^{T} \boldsymbol{\Sigma}^{-1}(\mathrm{y}-\boldsymbol{\mu})\right\}, \quad \boldsymbol{\Sigma}>0, \quad-\infty<x_{i}<\infty
$$

cujo logaritmo da função de verossimilhança é

$$
l(\mathrm{y}, \boldsymbol{\theta})=\ln L(\mathrm{y}, \boldsymbol{\theta})=\frac{10}{2} \log |2 \pi \Sigma|-\frac{1}{2} \sum_{i=1}^{10}\left(y_{i}-\boldsymbol{\mu}\right)^{T} \Sigma^{-1}\left(y_{i}-\boldsymbol{\mu}\right) ;
$$

2. usamos como valor inicial as estimativas obtidas a partir da parte completa dos dados, neste caso uma matriz de dimensão $(6 \times 4)$. Assim, o vetor de médias e a 
matriz de variâncias-covariâncias iniciais são:

$$
\begin{gathered}
\hat{\boldsymbol{\mu}}=\left(\begin{array}{l}
\boldsymbol{\mu}_{1} \\
\boldsymbol{\mu}_{2}
\end{array}\right)=\left(\begin{array}{l}
\mu_{1} \\
\mu_{2} \\
\mu_{3} \\
\mu_{4}
\end{array}\right)=\left(\begin{array}{l}
4,3987 \\
3,6500 \\
\frac{2,8988}{1,8243}
\end{array}\right) \\
\hat{\boldsymbol{\Sigma}}=\left(\begin{array}{l|l}
\boldsymbol{\Sigma}_{11} & \boldsymbol{\Sigma}_{12} \\
\hline \boldsymbol{\Sigma}_{21} & \boldsymbol{\Sigma}_{22}
\end{array}\right)=\left(\begin{array}{lll|l}
2,1559 & 1,0600 & 1,0949 & 0,7999 \\
1,0600 & 0,7752 & 0,8065 & 0,4278 \\
1,0949 & 0,8065 & 2,0084 & 1,2426 \\
\hline 0,7999 & 0,4278 & 1,2426 & 1,1015
\end{array}\right),
\end{gathered}
$$

já particionadas de acordo com o exemplo. Em seguida calculamos $l_{1}(\mathrm{y}, \theta)$, segundo a expressão (3.14), resultando em $l_{1}(\mathrm{y}, \theta)=-0,9768$;

3. a imputação, via algoritmo EM, foi feita mediante a seguinte expressão

$$
\begin{aligned}
& \mathbb{E}\left(y_{4 i} / \mathrm{y}_{o i}=\left(y_{1 i}, y_{2 i}, y_{3 i}\right)\right)=\mu_{2}+\Sigma_{21} \Sigma_{11}^{-1}\left(\mathrm{y}_{o i}-\boldsymbol{\mu}_{1}\right), \text { ou seja, } \\
& \mathbb{E}\left(y_{47} /(6,035 ; 4,441 ; 3,839)\right)=1,8243+\Sigma_{21} \Sigma_{11}^{-1}\left(\left(\begin{array}{l}
6,035 \\
4,441 \\
3,839
\end{array}\right)-\left(\begin{array}{l}
4,3987 \\
3,6500 \\
2,8988
\end{array}\right)\right)=2,5141 \\
& \mathbb{E}\left(y_{48} /(5,519 ; 4,552 ; 3,254)\right)=1,8243+\Sigma_{21} \Sigma_{11}^{-1}\left(\left(\begin{array}{c}
5,519 \\
4,552 \\
3,254
\end{array}\right)-\left(\begin{array}{l}
4,3987 \\
3,6500 \\
2,8988
\end{array}\right)\right)=1,8878 \\
& \mathbb{E}\left(y_{49} /(3,890 ; 4,849 ; 4,684)\right)=1,8243+\Sigma_{21} \Sigma_{11}^{-1}\left(\left(\begin{array}{l}
3,890 \\
4,849 \\
4,684
\end{array}\right)-\left(\begin{array}{l}
4,3987 \\
3,6500 \\
2,8988
\end{array}\right)\right)=2,1839 \\
& \mathbb{E}\left(y_{410} /(6,309 ; 5,429 ; 5.742)\right)=1,8243+\Sigma_{21} \Sigma_{11}^{-1}\left(\left(\begin{array}{l}
6,309 \\
5.429 \\
5,742
\end{array}\right)-\left(\begin{array}{l}
4,3987 \\
3,6500 \\
2,8988
\end{array}\right)\right)
\end{aligned}
$$


onde,

$$
\Sigma_{21}=\left(\begin{array}{lll}
0,7999 & 0,4278 \quad 1,2426)
\end{array}\right.
$$

e

$$
\boldsymbol{\Sigma}_{11}^{-1}=\left(\begin{array}{ccc}
1,4157 & -1,9458 & 0,0096 \\
-1,9458 & 4,8900 & -0,9029 \\
0,0096 & -0,9029 & 0,8553
\end{array}\right) \text {. }
$$

Logo, a matriz com dados imputados no primeiro passo é

$$
\mathbf{Y}=\left(\begin{array}{llll}
6,669 & 4,376 & 4,994 & 3,539 \\
4,937 & 4,676 & 2,996 & 1,821 \\
5,285 & 4,252 & 2,697 & 0,775 \\
1,977 & 2,388 & 2,278 & 0,904 \\
3,885 & 2,594 & 0,441 & 1,035 \\
3,639 & 3,614 & 3,987 & 2,872 \\
6,035 & 4,441 & 3,839 & 2,514 \\
5,519 & 4,552 & 3,254 & 1,888 \\
3,890 & 4,849 & 4,684 & 2,184 \\
6,309 & 5,429 & 5,742 & 3,322
\end{array}\right) ;
$$

4. repetimos todos os passos até convergir. Neste caso, a convergência foi obtida em dois passos, sendo o vetor de médias e a matriz de variâncias-covariâncias no segundo passo iguais a

$$
\hat{\boldsymbol{\mu}}=\left(\begin{array}{l}
\boldsymbol{\mu}_{1} \\
\boldsymbol{\mu}_{2}
\end{array}\right)=\left(\begin{array}{c}
\mu_{1} \\
\mu_{2} \\
\frac{\mu_{3}}{\mu_{4}}
\end{array}\right)=\left(\begin{array}{c}
4,8145 \\
4,1171 \\
\frac{3,4912}{2,0854}
\end{array}\right)
$$




$$
\hat{\Sigma}=\left(\begin{array}{c|c}
\Sigma_{11} & \Sigma_{12} \\
\hline \Sigma_{21} & \Sigma_{22}
\end{array}\right)=\left(\begin{array}{lll|l}
1,9047 & 0,9511 & 1,0563 & 0,7592 \\
0,9511 & 0,8511 & 1,0334 & 0,5046 \\
1,0563 & 1,0334 & 2,0821 & 1,1480 \\
\hline 0,7592 & 0,5046 & 1,1480 & 0,8779
\end{array}\right)
$$

e o valor de $l_{2}(\mathrm{y}, \theta)=1, \$ 964$, onde $\left|l_{1}-l_{2}\right|=2,8732$, que ainda é maior do que 0,001. Então

$$
\begin{aligned}
& \mathbb{E}\left(y_{47} /(6,035 ; 4,441 ; 3,839)\right)=2,0854+\Sigma_{21} \Sigma_{11}^{-1}\left(\left(\begin{array}{l}
6,035 \\
4,441 \\
3,839
\end{array}\right)-\left(\begin{array}{l}
4,8145 \\
4,1171 \\
3,4912
\end{array}\right)\right)=2,514 \\
& \mathbb{E}\left(y_{48} /(5,519 ; 4,552 ; 3,254)\right)=2,0854+\Sigma_{21} \Sigma_{11}^{-1}\left(\left(\begin{array}{c}
5,519 \\
4,552 \\
3,254
\end{array}\right)-\left(\begin{array}{l}
4,8145 \\
4,1171 \\
3,4912
\end{array}\right)\right)=1,888 \\
& \mathbb{E}\left(y_{49} /(3,890 ; 4,849 ; 4,684)\right)=2,0854+\Sigma_{21} \Sigma_{11}^{-1}\left(\left(\begin{array}{c}
3,890 \\
4,849 \\
4,684
\end{array}\right)-\left(\begin{array}{c}
4,8145 \\
4,1171 \\
3,4912
\end{array}\right)\right) \\
& \mathbb{E}\left(y_{410} /(6,309 ; 5,429 ; 5,742)\right)=2,0854+\Sigma_{21} \Sigma_{11}^{-1}\left(\left(\begin{array}{c}
6,309 \\
5,429 \\
5,742
\end{array}\right)-\left(\begin{array}{c}
4,8145 \\
4,1171 \\
3,4912
\end{array}\right)\right)
\end{aligned}
$$

onde,

$$
\Sigma_{21}=(0,7592 \quad 0,5046 \quad 1,1480)
$$

e

$$
\Sigma_{11}^{-1}=\left(\begin{array}{ccc}
1,2047 & -1,5203 & 0,1434 \\
-1,5203 & 4,8755 & -1,6485 \\
0,1434 & -1,6485 & 1,2258
\end{array}\right)
$$


Assim, a matriz com os dados imputados resultou em

$$
\mathbf{Y}=\left(\begin{array}{cccc}
6,669 & 4,376 & 4,994 & 3,539 \\
4,937 & 4,676 & 2,996 & 1,821 \\
5,285 & 4,252 & 2,697 & 0,775 \\
1,977 & 2,388 & 2,278 & 0,904 \\
3,885 & 2,594 & 0,441 & 1,035 \\
3,639 & 3,614 & 3,987 & 2,872 \\
6,035 & 4,441 & 3,839 & 2,514 \\
5,519 & 4,552 & 3,254 & 1,888 \\
3,890 & 4,849 & 4,684 & 2,184 \\
6,309 & 5,429 & 5,742 & 3,322
\end{array}\right) .
$$




\section{Capítulo 4}

\section{O bootstrap aplicado a dados incompletos}

\subsection{Introdução}

Neste capítulo descrevemos como a técnica bootstrap não paramétrica pode ser aplicada na presença de dados incompletos. Como já foi citado anteriormente, um método de imputação é usado para preencher os dados ausentes.

A questão que se coloca é: como estimar o erro padrão de um estimador $\hat{\theta}$, para dados incompletos?

Uma boa alternativa para responder a essa pergunta é aplicar a técnica bootstrap não paramétrica pois, além de não ser necessário conhecer a forma fechada do erro padrão, ainda tem como vantagem não depender do mecanismo de não resposta.

Da reduzida bibliografia sobre este tópico, pode-se citar Bello (1994), Efron (1994) e Shao e Sitter (1996). A literatura cita três diferentes alternativas para a utilização do bootstrap a dados incompletos. São elas:

1. efetuar a imputação aos dados ausentes na amostra original e depois aplicar a técnica bootstrap como se os dados fossem completos; 
2. selecionar amostras bootstrap sem levar em conta a presença de dados ausentes e depois efetuar a imputação em cada amostra bootstrap;

3. selecionar as amostras bootstrap, de modo que cada uma contenha a mesma proporção de dados observados e dados ausentes da amostra original e depois efetuar a imputação, garantindo desta maneira que cada réplica bootstrap selecionada esteja formada por dados da parte completa e da parte incompleta.

A primeira alternativa não é recomendável porque ignora o processo de imputação nas réplicas bootstrap. Os estimadores da variância são inconsistentes pois não é possível capturar a inflação da variância devida à imputação.

O ideal seria que o conjunto de dados bootstrap fosse também imputado na mesma forma como foi o conjunto dos dados originais.

O inconveniente da segunda alternativa é que as amostras bootstrap são tiradas diretamente, na forma usual, a partir dos dados originais incompletos. Nesse caso pode ocorrer a seleção de alguma amostra formada unicamente pela parte incompleta, não sendo possível assim, fazer a imputação. Por outro lado pode acontecer também a seleção de uma réplica composta somente por dados completos e isto implicaria em que nem todas as amostras bootstrap tivessem valores imputados.

A opção pela terceira alternativa garante que todas as réplicas tenham a mesma proporção de dados ausentes da amostra original, representando melhor a distribuição empírica dos dados.

Pelas razões acima expostas, os resultados de simulação apresentados adiante neste capítulo foram obtidos com a aplicação da terceira alternativa, cujo algoritmo está descrito na próxima seção. 


\subsection{Algoritmo bootstrap para dados incompletos}

No algoritmo bootstrap original, o passo 1 é selecionar $B$ amostras independentes com reposição. Davison e Hinkley (1992) implementaram o passo 1 do algoritmo bootstrap quando se têm dados faltantes. Eles propõem um método chamado Método Bootstrap Proporcional, no qual a proporção de dados completos e incompletos aparecem em cada amostra bootstrap, garantindo assim que:

1. cada amostra bootstrap seja incompleta de modo que representem completamente os dados incompletos originais;

2. permita o uso de alguma técnica de imputação de forma eficiente para todas as amostras bootstrap;

3. de certa forma, controle a proporção de valores ausentes da amostra bootstrap.

Assim, a implementação do passo 1 no algoritmo bootstrap para dados incompletos é feita, primeiro particionando os dados originais nas partes observada e ausente, ou seja, $\mathrm{y}=\left(\mathbf{y}_{\mathbf{o}}, \mathrm{y}_{\mathrm{a}}\right)$, de modo que existam $n_{1}$ casos completos e $n_{2}$ casos incompletos, onde $n=n_{1}+n_{2}$. A seguir seleciona-se uma amostra bootstrap de cada uma das partes. A reunião dessas duas amostras forma a réplica bootstrap, que sofrerá a imputação. Esquematizando, o passo 1 do algoritmo original, descrito na Seção 2.2.1 é substituído por

1 (a) selecionar uma amostra aleatória com reposição de tamanho $n_{1}$ de $\mathbf{y}_{\mathbf{o}}$;

(b) selecionar uma amostra aleatória com reposição de tamanho $n_{2}$ de $\mathbf{y}_{\mathbf{a}}$;

(c) juntar as amostras selecionadas em (a) e (b) para formar uma réplica bootstrap $\mathrm{y}^{* 1}=\left(\mathrm{y}_{\mathrm{o}}^{* 1}, \mathrm{y}_{\mathrm{a}}^{* 1}\right)$

(d) repetir os procedimentos em (a), (b) e (c), $B$ vezes; 
(e) fazer a imputação em $\mathrm{y}^{* 1} \cdot \mathrm{y}^{* 2}, \ldots, \mathrm{y}^{* \mathrm{~B}}$, completando as amostras.

A partir daí os passos 2 e 3 do algoritmo original são seguidos.

\subsection{Resultados da simulação}

Nesta seção apresentamos os resultados de simulações feitas com o objetivo de estimar as componentes da variância em um modelo linear misto e também as variâncias dessas componentes. As simulações foram feitas no S-PLUS de acordo com o seguinte critério.

Foram geradas 2000 amostras de dados $y_{i}=\left(y_{1 i}, y_{2 i}\right), i=1,2, \ldots, n$, de cada uma das formas:

1. Modelo linear misto: geramos $n$ pares de observações segundo o modelo linear misto usual com dois fatores, no qual o tempo é o fator fixo e o indivíduo o fator aleatório, ou seja,

$$
y_{t i}=\mu+\alpha_{t}+b_{i}+e_{t i}
$$

com $T=2, \mu=0, \alpha_{1}=0$ e $\alpha_{2}=0$, isto é, uma amostra painel com dois instantes.

Foram consideradas três combinações das componentes da variância:

$$
\begin{array}{llll}
\text { - } \sigma^{2}=4 \quad, \quad \sigma_{b}^{2}=4 & (\rho=0,5) \\
\text { - } \sigma^{2}=16 \quad, \quad \sigma_{b}^{2}=4 & (\rho=0,2) \\
\text { - } \sigma^{2}=4 \quad, \quad \sigma_{b}^{2}=16 & (\rho=0,8)
\end{array}
$$

2. Distribuição normal bivariada: geramos amostras com $n$ pares de dados independentes de uma distribuição normal bivariada. Foram consideradas as três matrizes de variâncias-covariâncias correspondentes às mesmas três combinações das componentes da variância no modelo linear misto, ou seja, 


$$
\begin{aligned}
& \text { - } \Sigma_{1}=\left(\begin{array}{ll}
8 & 4 \\
4 & 8
\end{array}\right) \\
& \text { - } \Sigma_{2}=\left(\begin{array}{ll}
20 & 4 \\
4 & 20
\end{array}\right) \\
& \text { - } \Sigma_{3}=\left(\begin{array}{ll}
20 & 16 \\
16 & 20
\end{array}\right)
\end{aligned}
$$

Nos três casos o vetor de médias foi fixado como $\boldsymbol{\mu}^{T}=(0,0)$.

Além das variâncias, mais dois fatores foram variados

a) o tamanho das amostras, fixados em $n=30$ e $n=100$;

b) a proporção de dados ausentes: as amostras foram geradas completas para avaliação de suas estimativas nessas condições. Para avaliação com dados incompletos, foram descartados aleatoriamente $p=20 \%$ e $p=40 \%$ da amostra.

Somente os dados do segundo instante é que ficaram sujeitos a ausência de informação.

A estimação das componentes da variância e de suas variâncias foi obtida através:

1. da expressão da variância dos estimadores com dados completos;

2. da técnica bootstrap com dados completos;

3. da técnica bootstrap aplicada a dados incompletos que sofreram imputação sob
a) o BLUP (Best Linear Unbiased Predictor);
b) o método de substituição da média (MSM);
c) o algoritmo EM;
d) o método das componentes principais de Dear (DPC). 
Para qualquer combinação. quando o bootstrap completo ou incompleto foi usado, foram selecionadas 200 amostras bootstrap.

As amostras foram geradas sob os dois modelos (misto e normal bivariada), pois havia o receio de que a geração sob o modelo misto pudesse privilegiar o BLUP, uma vez que, dado o modelo misto, ele é o melhor no sentido de ser linear, não viesado, de erro quadrático médio mínimo.

Os resultados obtidos estão apresentados em 17 tabelas.

As Tabelas 4.3.1, 4.3.2, 4.3.3 e 4.3.4 mostram as médias e os desvios padrão das 2000 estimativas das componentes da variância, no modelo linear misto. As Tabelas 4.3.1 e 4.3.2 contêm os resultados para $n=30$ e as Tabelas 4.3 .3 e 4.3 .4 , para $n=100$. As Tabelas 4.3 .1 e 4.3 .3 correspondem a $p=20 \%$ e as Tabelas 4.3 .2 e 4.3 .4 a $p=40 \%$. Para calcular os resultados obtidos pela expressão dos estimadores foram usadas as fórmulas (3.9) e (3.10) do Capítulo 3. Os valores constantes nas quatro colunas das tabelas foram obtidos, respectivamente, pelas seguintes expressões:

$$
\begin{aligned}
& \overline{\hat{\sigma}}^{2}=\frac{1}{2000} \sum_{i=1}^{2000}\left(\hat{\sigma}_{i}^{2}\right) \\
& \hat{\sigma}_{b}^{2}=\frac{1}{2000} \sum_{i=1}^{2000}\left(\hat{\sigma}_{b_{i}}^{2}\right) \\
& \hat{S}=\left\{\frac{1}{1999} \sum_{i=1}^{2000}\left(\hat{\sigma}_{i}^{2}-\overline{\hat{\sigma}}^{2}\right)^{2}\right\}^{1 / 2} \\
& \hat{S}_{b}=\left\{\frac{1}{1999} \sum_{i=1}^{2000}\left(\hat{\sigma}_{b_{i}}^{2}-\overline{\hat{\sigma}}_{b}^{2}\right)^{2}\right\}^{1 / 2} .
\end{aligned}
$$

As Tabelas 4.3.1a, 4.3.2a, 4.3.3a e 4.3.4a mostram as médias e desvios padrão das 2000 estimativas das variâncias das componentes da variância no modelo linear misto . A combinação entre as tabelas é análoga àquela feita nas Tabelas 4.3.1, 4.3.2, 4.3.3 e 4.3.4. Os resultados referentes à expressão foram obtidos pela aplicação de (3.11) e (3.12) do 
Capítulo 3. Para os cálculos dos números apresentados nestas tabelas foram usadas

$$
\begin{aligned}
\overline{\operatorname{Var}}\left(\hat{\sigma}^{2}\right) & =\frac{1}{2000} \sum_{i=1}^{2000}\left[\hat{\operatorname{Var}}\left(\hat{\sigma}_{i}^{2}\right)\right] \\
\overline{\operatorname{Var}\left(\hat{\sigma}_{b}^{2}\right)} & =\frac{1}{2000} \sum_{i=1}^{2000}\left[\hat{\left.\operatorname{Var}\left(\hat{\sigma}_{b_{i}}^{2}\right)\right]}\right. \\
\hat{S}_{\text {var }} & =\left\{\frac{1}{1999} \sum_{i=1}^{2000}\left[\hat{\operatorname{Var}}\left(\hat{\sigma}_{i}^{2}\right)-\overline{\operatorname{Var}}\left(\hat{\sigma}^{2}\right)\right]^{2}\right\}^{1 / 2} \\
\hat{S}_{\text {var }_{b}} & =\left\{\frac{1}{1999} \sum_{i=1}^{2000}\left[\hat{\operatorname{Var}}\left(\hat{\sigma}_{b_{i}}^{2}\right)-\overline{\operatorname{Var}}\left(\hat{\sigma}_{b}^{2}\right)\right]^{2}\right\}^{1 / 2} .
\end{aligned}
$$

As Tabelas 4.3.5, 4.3.6, 4.3.7, 4.3.8 e 4.3.5a, 4.3.6a, 4.3.7a, 4.3.8a são análogas às anteriores só que correspondem aos dados gerados pela distribuição normal bivariada.

A Tabela 4.3.9 mostra os coeficientes de variação obtidos a partir das Tabelas 4.3.1,

\subsection{2, 4.3.3 e 4.3.4.}

Dessas simulações, pudemos observar o que segue.

Quando $\rho=0,5, \hat{S}, \hat{S}_{b}, \hat{S}_{\text {var }}$ e $\hat{S}_{\text {var }}$ são menores do que quando $\rho=0,2$ e $\rho=0,8$, sugerindo que os desvios padrão aumentam quando $\rho$ se afasta de 0,5 .

Quando $n$ cresce, $\hat{S}$ e $\hat{S}_{b}$ decrescem, enquanto que $\hat{\sigma}^{2}$ e $\hat{\sigma}_{b}^{2}$ são mais próximos dos valores originais. Da mesma forma, podemos observar que $\overline{\operatorname{Var}}\left(\hat{\sigma}^{2}\right), \overline{\operatorname{Var}}\left(\hat{\sigma}_{b}^{2}\right), \hat{S}_{\text {var }}$ e $\hat{S}_{\text {var }_{b}}$ decrescem a medida que $n$ cresce.

O estimador da variância do efeito aleatório dado pela expressão quando $\rho=0,5$ subestima o parâmetro.

Para $n=100$, os resultados obtidos pela expressão e pelo bootstrap completo são muito similares, somente o desvio padrão da variância de $\hat{\sigma}_{b}^{2}$ é maior para o bootstrap completo em comparação com a expressão.

Para dados incompletos, as componentes da variância são subestimadas qualquer que seja o método de imputação utilizado. Isso já era esperado pois todos os métodos aqui usados são determinísticos e não consideram a inflação da variância devida à falta de 
informações. A idéia de usar esses métodos foi para poder avaliar o tamanho dessa subestimação. Cabe ressaltar que a imputação determinística não aumenta muito a quantidade de computação sobre o bootstrap completo. Já para a imputação aleatória, tem-se que gerar os resíduos aleatórios para cada dado, causando um expressivo aumento de computação.

Ainda com relação à simulação, quando a proporção de dados ausentes aumenta, os resultados pioram, as médias se distanciam dos parâmetros e os desvios padrão aumentam.

A Tabela 4.3.9 mostra os coeficientes de variação calculados sobre as Tabelas 4.3.1, 4.3 .2 , 4.3.3 e 4.3.4, para os quatro métodos de imputação, para $p=20 \%$ e $p=40 \%$ e para $n=30$ e $n=100$. As três repetições em cada casela correspondem aos três valores de $\rho, 0,5,0,2$ e 0,8 , respectivamente. Pode-se perceber que os maiores coeficientes de variação ocorrem quando $\rho=0,2$, para estimar $\sigma_{b}^{2}$ e são substancialmente maiores do que os outros. O BLUP e o método da média têm comportamentos semelhantes e melhores do que o algoritmo EM e do que o método de componentes principais, uma vez que apresentam, em geral, coeficientes de variação menores do que os dois últimos. Isso ocorre pois, em geral, as médias do algoritmo EM e do componentes principais são menores do que as médias dos outros dois métodos, ou seja, eles subestimam mais as componentes da variância.

Com relação às Tabelas 4.3.1a, 4.3.2a, 4.3.3a e 4.3.4a as médias das variâncias de $\hat{\sigma}^{2} \mathrm{e}$ $\hat{\sigma}_{b}^{2}$ são maiores quando se usa a expressão dos estimadores, com exceção da variância de $\hat{\sigma}^{2}$ quando $\rho=0,8$ e $n=100$ e quando o método de componentes principais é usado com $\rho=0,5$ e $n=100$.

Quando $\rho=0,2$ e $n=30$, as médias e os desvios padrão da variância de $\hat{\sigma}_{b}^{2}$ são extremamente altos, mostrando que se perde muito na estimação quando $\sigma_{b}^{2}$ é bem menor do que $\sigma^{2}$. Isso talvez possa ser explicado porque $\sigma_{b}^{2}$ é um estimador truncado no zero e problemas costumam surgir para amostras pequenas quando a razão entre $\sigma_{b}^{2}$ e $\sigma^{2}$ é baixa. 
Quando os dados são gerados a partir de uma distribuição normal bivariada, os resultados são praticamente os mesmos, mostrando que o BLUP não foi privilegiado, o que dá certa robustez a esse método, quando se supõe o modelo linear misto. Nesta simulação somente dois instantes foram considerados. O mesmo pode não ocorrer se mais instantes tivessem sido observados, pois o modelo linear misto impõe uma estrutura de correlação uniforme no tempo, que aqui não aparece pois consideramos somente dois instantes. 
Tabela 4.3.1: Médias e desvios padrão das 2000 estimativas das componentes da variância no modelo linear misto $(n=30, p=20 \%$ )

\begin{tabular}{|c|c|c|c|c|c|c|c|}
\hline$n$ & $\rho$ & MLM & Método & $\overline{\hat{\sigma}}^{2}$ & $\overline{\hat{\sigma}}_{b}^{2}$ & $\hat{S}$ & $\hat{S}_{b}$ \\
\hline \multirow{6}{*}{30} & \multirow{6}{*}{0,5} & \multirow{6}{*}{$\begin{aligned} e_{t i} & \sim N(0,4) \\
b_{i} & \sim N(0,4)\end{aligned}$} & expressão & 4,0198 & 3,7689 & 1,0876 & 1,5844 \\
\hline & & & boots completo & 3,7865 & 3,9765 & 1,0098 & 1,4567 \\
\hline & & & BLUP $(20 \%)$ & 3,8978 & 3,4436 & 1,2175 & 1,4839 \\
\hline & & & Média(20\%) & 4,0065 & 3,8604 & 1,4637 & 1,8163 \\
\hline & & & $\operatorname{EM}(20 \%)$ & 3,2976 & 3,8654 & 1,3943 & 1,9632 \\
\hline & & & $\mathrm{CP}(20 \%)$ & 3,6432 & 3,5486 & 1,5375 & 1,7391 \\
\hline \multirow{6}{*}{30} & \multirow{6}{*}{0,2} & \multirow{6}{*}{$\begin{array}{l}e_{t i} \sim N(0,16) \\
b_{i} \sim N(0,4)\end{array}$} & expressão & 16,1114 & 4,0098 & 4,5647 & 3,9845 \\
\hline & & & boots completo & 15,3413 & 4,8639 & 4,2639 & 2,8712 \\
\hline & & & BLUP(20\%) & 14,6591 & 3,8536 & 5,0967 & 3,4853 \\
\hline & & & Média(20\%) & 14,3976 & 3,7849 & 5,2354 & 3,3642 \\
\hline & & & $\operatorname{EM}(20 \%)$ & 13,3205 & 3,6857 & 5,4365 & 4,3478 \\
\hline & & & C.P $(20 \%)$ & 15,2654 & 3,1829 & 5,2734 & 3,6459 \\
\hline \multirow{6}{*}{30} & \multirow{6}{*}{0,8} & \multirow{6}{*}{$\begin{array}{l}e_{t i} \sim N(0,4) \\
b_{i} \sim N(0,16)\end{array}$} & expressão & 4,1256 & 16,0003 & 1,3579 & 5,3707 \\
\hline & & & boots completo & 3,9111 & 15,8751 & 1,0397 & 4,1678 \\
\hline & & & BLUP(20\%) & 3,8654 & 14,9701 & 1,7483 & 4,4790 \\
\hline & & & Média(20\%) & 4,0051 & 14,6981 & 1,4320 & 5,1308 \\
\hline & & & $\operatorname{EM}(20 \%)$ & 3,6395 & 13,9578 & 1,3079 & 6,0130 \\
\hline & & & $\mathrm{CP}(20 \%)$ & 3,6542 & 15,3618 & 1,1943 & 6,0037 \\
\hline
\end{tabular}


Tabela 4.3.2: Médias e desvios padrão das 2000 estimativas das componentes da variância no modelo linear misto $(n=30, p=40 \%)$

\begin{tabular}{|c|c|c|c|c|c|c|c|}
\hline$n$ & $\rho$ & MLM & Método & $\overline{\hat{\sigma}}^{2}$ & $\hat{\sigma}_{b}^{2}$ & $\hat{S}$ & $\hat{S}_{b}$ \\
\hline \multirow{6}{*}{30} & \multirow{6}{*}{0,5} & \multirow{6}{*}{$\begin{array}{l}e_{t i} \sim N(0,4) \\
b_{i} \sim N(0,4)\end{array}$} & expressão & 4,0198 & 3,7689 & 1,0876 & 1,5844 \\
\hline & & & boots completo & 3,7865 & 3,9765 & 1,0098 & 1,4567 \\
\hline & & & BLUP $(40 \%)$ & 3,6565 & 3,2356 & 1,5647 & 1,9865 \\
\hline & & & Média(40\%) & 3,8743 & 3,6578 & 1,6580 & 1,9148 \\
\hline & & & $\operatorname{EM}(40 \%)$ & 3,1865 & 3,5709 & 1,9541 & 1,6163 \\
\hline & & & $\mathrm{CP}(40 \%)$ & 3,5849 & 3,3980 & 1,7344 & 1,5568 \\
\hline \multirow{6}{*}{30} & \multirow{6}{*}{0,2} & \multirow{6}{*}{$\begin{array}{l}e_{t i} \sim N(0,16) \\
b_{i} \sim N(0,4)\end{array}$} & expressão & 16,1114 & 4,0098 & 4,5647 & 3,9845 \\
\hline & & & boots completo & 15,3413 & 4,8639 & 4,2639 & 2,8712 \\
\hline & & & BLUP(40\%) & 13,9876 & 3,6903 & 5,2986 & 3,9107 \\
\hline & & & Média(40\%) & 13,8507 & 3,7009 & 5,1973 & 3,4521 \\
\hline & & & $\operatorname{EM}(40 \%)$ & 14,0017 & 3,6542 & 5,5819 & 3,8129 \\
\hline & & & $\mathrm{CP}(40 \%)$ & 14,8639 & 3,6937 & 5,7842 & 3,4902 \\
\hline \multirow{6}{*}{30} & \multirow{6}{*}{0,8} & \multirow{6}{*}{$\begin{array}{l}e_{t i} \sim N(0,4) \\
b_{i} \sim N(0,16)\end{array}$} & expressão & 4,1256 & 16,0003 & 1,3579 & 5,3707 \\
\hline & & & boots completo & 3,9111 & 15,8751 & 1,0397 & 4,9678 \\
\hline & & & BLUP $(40 \%)$ & 3,5499 & 14,6791 & 2,0019 & 5,0289 \\
\hline & & & Média(40\%) & 3,9869 & 14,2746 & 1,5891 & 5,3537 \\
\hline & & & $\operatorname{EM}(40 \%)$ & 3,5885 & 14,2310 & 2,1729 & 5,8430 \\
\hline & & & $\mathrm{CP}(40 \%)$ & 3,6693 & 14,8590 & 2,1308 & 5,4778 \\
\hline
\end{tabular}


Tabela 4.3.1a: Médias e desvios padrão das 2000 estimativas das variâncias das componentes da variância no modelo linear misto $(n=30, p=20 \%)$

\begin{tabular}{|c|c|c|c|c|c|c|c|}
\hline$n$ & $\rho$ & MLM & Método & $\overline{\operatorname{Var}}\left(\hat{\sigma}^{2}\right)$ & $\operatorname{Var}\left(\hat{\sigma}_{b}^{2}\right)$ & $\hat{S}_{\text {var }}$ & $\hat{S}_{\text {var }_{b}}$ \\
\hline \multirow{6}{*}{30} & \multirow{6}{*}{0,5} & \multirow{6}{*}{$\begin{array}{l}e_{t i} \sim N(0,4) \\
b_{i} \sim N(0,4)\end{array}$} & expressão & 1,5217 & 2,8365 & 0,8048 & 1,2532 \\
\hline & & & boots completo & 1,0329 & 2,1837 & 0,7069 & 1,4276 \\
\hline & & & BLUP(20\%) & 1,2826 & 1,9308 & 0,8754 & 1,7139 \\
\hline & & & Média(20\%) & 0,9937 & 1,6869 & 0,7548 & 1,5839 \\
\hline & & & $\operatorname{EM}(20 \%)$ & 0,7134 & 1,9196 & 0,5416 & 1,8314 \\
\hline & & & $\mathrm{CP}(20 \%)$ & 1,2581 & 1,7762 & 0,5391 & 1,9224 \\
\hline \multirow{6}{*}{30} & \multirow{6}{*}{0,2} & \multirow{6}{*}{$\begin{array}{l}e_{t i} \sim N(0,16) \\
b_{i} \sim N(0,4)\end{array}$} & expressão & 17,5098 & 14,5362 & 10,7912 & 6,6718 \\
\hline & & & boots completo & 16,6054 & 10,0098 & 11,2819 & 7,3290 \\
\hline & & & BLUP $(20 \%)$ & 13,9078 & 10,7639 & 11,8528 & 7,1789 \\
\hline & & & Média(20\%) & 13,8439 & 9,5320 & 12,6627 & 6,6463 \\
\hline & & & $\operatorname{EM}(20 \%)$ & 12,7482 & 9,6845 & 12,7953 & 7,4729 \\
\hline & & & $\mathrm{CP}(20 \%)$ & 14,3769 & 10,5569 & 11,8709 & 6,6307 \\
\hline \multirow{6}{*}{30} & \multirow{6}{*}{0,8} & \multirow{6}{*}{$\begin{array}{l}e_{t i} \sim N(0,4) \\
b_{i} \sim N(0,16)\end{array}$} & expressão & 1,2110 & 22,3685 & 0,8176 & 14,6829 \\
\hline & & & boots completo & 0,9976 & 20,4821 & 0,8955 & 14,7613 \\
\hline & & & BLUP $(20 \%)$ & 1,0097 & 17,6926 & 0,7540 & 15,0406 \\
\hline & & & Média(20\%) & 1,1103 & 16,1639 & 1,2947 & 14,8638 \\
\hline & & & $\operatorname{EM}(20 \%)$ & 0,9475 & 18,7971 & 0,9472 & 17,5748 \\
\hline & & & $\mathrm{CP}(20 \%)$ & 1,1029 & 17,6780 & 0,8593 & 16,5455 \\
\hline
\end{tabular}


Tabela 4.3.2a: Médias e desvios padrão das 2000 estimativas das variâncias das componentes da variância no modelo linear misto $(n=30, p=40 \%)$

\begin{tabular}{|c|c|c|c|c|c|c|c|}
\hline$n$ & $\rho$ & MLM & Método & $\overline{\operatorname{Var}}\left(\hat{\sigma}^{2}\right)$ & $\overline{\operatorname{Var}}\left(\hat{\sigma}_{b}^{2}\right)$ & $\hat{S}_{\text {var }}$ & $\hat{S}_{\text {var }_{b}}$ \\
\hline \multirow{6}{*}{30} & \multirow{6}{*}{0,5} & \multirow{6}{*}{$\begin{array}{l}e_{t i} \sim N(0,4) \\
b_{i} \sim N(0,4)\end{array}$} & expressão & 1,5217 & 2,8365 & 0,8048 & 1,2532 \\
\hline & & & boots completo & 1,0329 & 2,1837 & 0,7069 & 1,4276 \\
\hline & & & BLUP(40\%) & 1,1356 & 1,6854 & 0,7659 & 1,6730 \\
\hline & & & Média(40\%) & 0,7967 & 1,5946 & 0,6865 & 1,4369 \\
\hline & & & $\operatorname{EM}(40 \%)$ & 0,6389 & 1,8754 & 0,4930 & 1,7649 \\
\hline & & & $\mathrm{CP}(40 \%)$ & 1,1356 & 1,6589 & 0,4789 & 1,6740 \\
\hline \multirow{6}{*}{30} & \multirow{6}{*}{0,2} & \multirow{6}{*}{$\begin{array}{l}e_{t i} \sim N(0,16) \\
b_{i} \sim N(0,4)\end{array}$} & expressão & 17,5098 & 14,5362 & 10,7912 & 6,6718 \\
\hline & & & boots completo & 16,6054 & 10,0098 & 11,2819 & 7,3290 \\
\hline & & & BLUP $(40 \%)$ & 12,6589 & 9,3536 & 11,1570 & 7,0098 \\
\hline & & & Média(40\%) & 12,9769 & 8,6784 & 11,7683 & 6,0096 \\
\hline & & & $\operatorname{EM}(40 \%)$ & 12,0124 & 8,8098 & 12,3541 & 6,3903 \\
\hline & & & $\mathrm{CP}(40 \%)$ & 13,4782 & 8,2908 & 10,4857 & 6,8082 \\
\hline \multirow{6}{*}{30} & \multirow{6}{*}{0,8} & \multirow{6}{*}{$\begin{array}{l}e_{t i} \sim N(0,4) \\
b_{i} \sim N(0,16)\end{array}$} & expressão & 1,2110 & 22,3685 & 0,8176 & 14,6829 \\
\hline & & & boots completo & 0,9976 & 20,4821 & 0,8955 & 14,7613 \\
\hline & & & BLUP $(40 \%)$ & 0,9765 & 16,1432 & 0,6548 & 15,7438 \\
\hline & & & Média(40\%) & 1,0975 & 15,5325 & 1,1838 & 14,0095 \\
\hline & & & $\operatorname{EM}(40 \%)$ & 0,8659 & 17,8751 & 0,7849 & 16,6850 \\
\hline & & & $\mathrm{CP}(40 \%)$ & 0,9976 & 15,3895 & 0,8997 & 14,2819 \\
\hline
\end{tabular}


Tabela 4.3.3: Médias e desvios padrão das 2000 estimativas das componentes da variância no modelo linear misto $(n=100, p=20 \%)$

\begin{tabular}{|c|c|c|c|c|c|c|c|}
\hline$n$ & $\rho$ & MLM & Método & $\overline{\hat{\sigma}}^{2}$ & $\overline{\hat{\sigma}}_{b}^{2}$ & $\hat{S}$ & $\hat{S}_{b}$ \\
\hline \multirow{6}{*}{100} & \multirow{6}{*}{0,5} & \multirow{6}{*}{$\begin{array}{l}e_{t i} \sim N(0,4) \\
b_{i} \sim N(0,4)\end{array}$} & expressão & 4,0123 & 3,8943 & 0,5427 & 0,9009 \\
\hline & & & boots completo & 4,0124 & 3,9278 & 0,5354 & 0,8189 \\
\hline & & & BLUP $(20 \%)$ & 4,0007 & 3,7438 & 0,7469 & 1,1145 \\
\hline & & & Média(20\%) & 3,9034 & 3,7946 & 0,7432 & 1,0628 \\
\hline & & & $\operatorname{EM}(20 \%)$ & 3,5289 & 3,3121 & 0,7951 & 1,2439 \\
\hline & & & $\mathrm{CP}(20 \%)$ & 3,7947 & 3,6729 & 1,0035 & 1,1372 \\
\hline \multirow{6}{*}{100} & \multirow{6}{*}{0,2} & \multirow{6}{*}{$\begin{array}{l}e_{t i} \sim N(0,16) \\
b_{i} \sim N(0,4)\end{array}$} & expressão & 15,9984 & 4,0382 & 2,1836 & 1,9260 \\
\hline & & & boots completo & 15,7485 & 3,9538 & 2,1538 & 1,9362 \\
\hline & & & BLUP(20\%) & 14,4285 & 3,6389 & 2,7369 & 2,2374 \\
\hline & & & Média(20\%) & 14,6258 & 3,9943 & 2,8356 & 2,3426 \\
\hline & & & $\operatorname{EM}(20 \%)$ & 14,0124 & 3,3538 & 3,1192 & 2,7284 \\
\hline & & & $\mathrm{CP}(20 \%)$ & 14,8372 & 3,5037 & 2,5278 & 2,5128 \\
\hline \multirow{6}{*}{100} & \multirow{6}{*}{0,8} & \multirow{6}{*}{$\begin{array}{l}e_{t i} \sim N(0,4) \\
b_{i} \sim N(0,16)\end{array}$} & expressão & 3,9954 & 16,0118 & 0,6378 & 2,5210 \\
\hline & & & boots completo & 3,9528 & 15,9350 & 0,5638 & 2,4529 \\
\hline & & & BLUP(20\%) & 3,7638 & 14,9935 & 1,1730 & 3,1538 \\
\hline & & & Média(20\%) & 3,6528 & 14,3849 & 1,3426 & 3,5931 \\
\hline & & & $\operatorname{EM}(20 \%)$ & 3,3729 & 14,7019 & 0,9982 & 3,4372 \\
\hline & & & $\mathrm{CP}(20 \%)$ & 3,4729 & 15,5038 & 1,9165 & 3,8258 \\
\hline
\end{tabular}


Tabela 4.3.4: Médias e desvios padrão das 2000 estimativas das componentes da variância no modelo linear misto $(n=100, p=40 \%)$

\begin{tabular}{|c|c|c|c|c|c|c|c|}
\hline$n$ & $\rho$ & MLM & Método & $\hat{\hat{\sigma}}^{2}$ & $\overline{\hat{\sigma}}_{b}^{2}$ & $\hat{S}$ & $\hat{S}_{b}$ \\
\hline 100 & 0,5 & $\begin{array}{l}e_{t i} \sim N(0,4) \\
b_{i} \sim N(0,4)\end{array}$ & $\begin{array}{l}\text { expressão } \\
\text { boots completo } \\
\text { BLUP }(40 \%) \\
\text { Média }(40 \%) \\
\operatorname{EM}(40 \%) \\
\text { CP }(40 \%)\end{array}$ & $\begin{array}{l}4,0123 \\
4,0124 \\
3,8428 \\
3,9146 \\
3,4278 \\
3,8256\end{array}$ & $\begin{array}{l}3,8943 \\
3,9278 \\
3,7481 \\
3,7258 \\
3,2438 \\
3,5749\end{array}$ & $\begin{array}{l}0,5427 \\
0,5354 \\
0,8475 \\
0,8642 \\
0,8137 \\
0,9867\end{array}$ & $\begin{array}{l}0,9009 \\
0,8189 \\
1,1629 \\
1,1538 \\
1,2648 \\
1,2091\end{array}$ \\
\hline 100 & 0,2 & $\begin{array}{l}e_{t i} \sim N(0,16) \\
b_{i} \sim N(0,4)\end{array}$ & $\begin{array}{l}\text { expressão } \\
\text { boots completo } \\
\text { BLUP }(40 \%) \\
\text { Média }(40 \%) \\
\operatorname{EM}(40 \%) \\
\operatorname{CP}(40 \%)\end{array}$ & $\begin{array}{l}15,9984 \\
15,7485 \\
14,4652 \\
14,5637 \\
13,9538 \\
14,6382\end{array}$ & $\begin{array}{l}4,0382 \\
3,9538 \\
3,6578 \\
3,8149 \\
3,1428 \\
3,2749\end{array}$ & $\begin{array}{l}2,1836 \\
2,1538 \\
2,8569 \\
3,1742 \\
3,1965 \\
3,1748\end{array}$ & $\begin{array}{l}1,9260 \\
1,9362 \\
2,2748 \\
2,6483 \\
2,9361 \\
2,6293\end{array}$ \\
\hline 100 & 0,8 & $\begin{array}{l}e_{t i} \sim N(0,4) \\
b_{i} \sim N(0,16)\end{array}$ & $\begin{array}{l}\text { expressão } \\
\text { boots completo } \\
\text { BLUP }(40 \%) \\
\text { Média }(40 \%) \\
\operatorname{EM}(40 \%) \\
\operatorname{CP}(40 \%)\end{array}$ & $\begin{array}{l}3,9954 \\
3,9528 \\
3,5389 \\
3,6729 \\
3,2098 \\
3,6452\end{array}$ & $\begin{array}{l}16,0118 \\
15,9350 \\
14,4751 \\
14,5694 \\
14,3627 \\
15,4318\end{array}$ & $\begin{array}{l}0,6378 \\
0,5638 \\
1,2593 \\
1,3619 \\
1,1283 \\
1,8749\end{array}$ & $\begin{array}{l}2,5210 \\
2,4529 \\
3,4195 \\
3,7935 \\
3,8354 \\
3,9627\end{array}$ \\
\hline
\end{tabular}


Tabela 4.3.3a: Médias e desvios padrão das 2000 estimativas das variâncias das componentes da variância no modelo linear misto $(n=100, p=20 \%)$

\begin{tabular}{|c|c|c|c|c|c|c|c|}
\hline$n$ & $\rho$ & MLM & Método & $\overline{\operatorname{Var}}\left(\hat{\sigma}^{2}\right)$ & $\overline{\operatorname{Var}}\left(\hat{\sigma}_{b}^{2}\right)$ & $\hat{S}_{\text {var }}$ & $\hat{S}_{\text {var }_{b}}$ \\
\hline \multirow{6}{*}{100} & \multirow{6}{*}{0,5} & \multirow{6}{*}{$\begin{array}{l}e_{t i} \sim N(0,4) \\
b_{i} \sim N(0,4)\end{array}$} & expressão & 0,3657 & 0,8356 & 0,1134 & 0,1866 \\
\hline & & & boots completo & 0,3498 & 0,7368 & 0,1005 & 0,2487 \\
\hline & & & BLUP(20\%) & 0,3274 & 0,6859 & 0,1358 & 0,3627 \\
\hline & & & Média(20\%) & 0,3465 & 0,6287 & 0,1634 & 0,3503 \\
\hline & & & $\operatorname{EM}(20 \%)$ & 0,2749 & 0,6054 & 0,1218 & 0,3119 \\
\hline & & & $\mathrm{CP}(20 \%)$ & 0,4984 & 0,6854 & 0,3164 & 0,3751 \\
\hline \multirow{6}{*}{100} & \multirow{6}{*}{0,2} & \multirow{6}{*}{$\begin{array}{l}e_{t i} \sim N(0,16) \\
b_{i} \sim N(0,4)\end{array}$} & expressão & 4,8638 & 4,1746 & 1,5738 & 0,9429 \\
\hline & & & boots completo & 4,7839 & 3,7093 & 1,9648 & 1,3428 \\
\hline & & & BLUP(20\%) & 4,1958 & 2,6209 & 2,2956 & 1,5941 \\
\hline & & & Média(20\%) & 4,2538 & 2,6374 & 2,3259 & 1,5983 \\
\hline & & & $\operatorname{EM}(20 \%)$ & 4,1743 & 2,7368 & 2,3090 & 1,6563 \\
\hline & & & $\mathrm{CP}(20 \%)$ & 4,8138 & 2,7864 & 2,8509 & 1,8349 \\
\hline \multirow{6}{*}{100} & \multirow{6}{*}{0,8} & \multirow{6}{*}{$\begin{array}{l}e_{t i} \sim N(0,4) \\
b_{i} \sim N(0,16)\end{array}$} & expressão & 0,3265 & 6,5829 & 0,1573 & 1,7461 \\
\hline & & & boots completo & 0,3021 & 6,3829 & 0,1537 & 2,5739 \\
\hline & & & BLUP(20\%) & 1,4083 & 5,8249 & 1,2761 & 3,1198 \\
\hline & & & Média(20\%) & 1,6352 & 5,3001 & 0,4738 & 3,1165 \\
\hline & & & $\operatorname{EM}(20 \%)$ & 1,2865 & 5,8965 & 0,3658 & 3,1968 \\
\hline & & & $\mathrm{CP}(20 \%)$ & 1,4869 & 5,6987 & 1,5814 & 3,3065 \\
\hline
\end{tabular}


Tabela 4.3.4a: Médias e desvios padrão das 2000 estimativas das variâncias das componentes da variância no modelo linear misto $(n=100, p=40 \%)$

\begin{tabular}{|c|c|c|c|c|c|c|c|}
\hline$n$ & $\rho$ & MLM & Método & $\overline{\operatorname{Var}}\left(\hat{\sigma}^{2}\right)$ & $\overline{\operatorname{Var}}\left(\hat{\sigma}_{b}^{2}\right)$ & $\hat{S}_{\text {var }}$ & $\hat{S}_{\text {var }_{b}}$ \\
\hline \multirow{6}{*}{100} & \multirow{6}{*}{0,5} & \multirow{6}{*}{$\begin{array}{l}e_{t i} \sim N(0.4) \\
b_{i} \sim N(0.4)\end{array}$} & expressão & 0,3657 & 0,8356 & 0,1134 & 0,1866 \\
\hline & & & boots completo & 0,3498 & 0,7368 & 0,1005 & 0,2487 \\
\hline & & & BLUP(40\%) & 0,3345 & 0,6436 & 0,1648 & 0,3362 \\
\hline & & & Média(40\%) & 0,3296 & 0,6264 & 0,1675 & 0,3482 \\
\hline & & & $\operatorname{EM}(40 \%)$ & 0,2468 & 0,4765 & 0,1763 & 0,2759 \\
\hline & & & $\mathrm{CP}(40 \%)$ & 0,3784 & 0,6256 & 0,1794 & 0,3268 \\
\hline \multirow{6}{*}{100} & \multirow{6}{*}{0,2} & \multirow{6}{*}{$\begin{array}{l}e_{t i} \sim N(0,16) \\
b_{i} \sim N(0.4)\end{array}$} & expressão & 4,8638 & 4,1746 & 1,5738 & 0,9429 \\
\hline & & & boots completo & 4,7839 & 3,7093 & 1,9648 & 1,3428 \\
\hline & & & BLUP(40\%) & 4,3358 & 2,6099 & 2,5300 & 1,7998 \\
\hline & & & Média(40\%) & 4,4998 & 2,5783 & 2,6146 & 1,6609 \\
\hline & & & $\operatorname{EM}(40 \%)$ & 4,5972 & 2,1742 & 2,4638 & 1,5748 \\
\hline & & & $\mathrm{CP}(40 \%)$ & 4,5738 & 2,3961 & 2,4328 & 1,4099 \\
\hline \multirow{6}{*}{100} & \multirow{6}{*}{0,8} & \multirow{6}{*}{$\begin{array}{l}e_{t i} \sim N(0.4) \\
b_{i} \sim N(0.16)\end{array}$} & expressão & 0,3265 & 6,5829 & 0,1573 & 1,7461 \\
\hline & & & boots completo & 0,3021 & 6,3829 & 0,1537 & 2,5739 \\
\hline & & & BLUP(40\%) & 1,2658 & 5,7012 & 1,1857 & 3,6148 \\
\hline & & & Média(40\%) & 1,2748 & 5,4538 & 0,9468 & 3,4658 \\
\hline & & & $\operatorname{EM}(40 \%)$ & 1,1547 & 5,5971 & 1,1005 & 3,4378 \\
\hline & & & $\mathrm{CP}(40 \%)$ & 1,2638 & 5,4091 & 1,0472 & 3,4291 \\
\hline
\end{tabular}


Tabela 4.3.5: Médias e desvios padrão das 2000 estimativas das componentes da variância na normal bivariada $(n=30, p=20 \%$ )

\begin{tabular}{|c|c|c|c|c|c|c|c|c|}
\hline$n$ & $\rho$ & Normal & & Método & $\overline{\hat{\sigma}}^{2}$ & $\hat{\sigma}_{b}^{2}$ & $\hat{S}$ & $\hat{S}_{b}$ \\
\hline \multirow{6}{*}{30} & \multirow{6}{*}{0,5} & \multirow{6}{*}{$\Sigma_{1}=$} & \multirow{6}{*}{$\left.\begin{array}{ll}8 & 4 \\
4 & 8\end{array}\right)$} & expressão & 4,0099 & 3,8988 & 1,1565 & 1,5487 \\
\hline & & & & boots completo & 3,9244 & 3,7415 & 1,2087 & 1,2509 \\
\hline & & & & BLUP(20\%) & 3,6495 & 3,5861 & 1,5974 & 1,8534 \\
\hline & & & & Média(20\%) & 3,9658 & 3,9570 & 1,5295 & 1,6123 \\
\hline & & & & $\operatorname{EM}(20 \%)$ & 3,8817 & 3,8148 & 1,5292 & 1,8765 \\
\hline & & & & $\mathrm{CP}(20 \%)$ & 3,6547 & 3,7961 & 1,3489 & 1,6743 \\
\hline \multirow{6}{*}{30} & \multirow{6}{*}{0,2} & \multirow{6}{*}{$\Sigma_{2}=$} & \multirow{6}{*}{$\left.\begin{array}{cc}20 & 4 \\
4 & 20\end{array}\right)$} & expressão & 16,0078 & 4,1289 & 4,6547 & 3,1289 \\
\hline & & & & boots completo & 15,1267 & 4,3759 & 4,3467 & 3,0019 \\
\hline & & & & BLUP(20\%) & 14,7321 & 3,7349 & 5,1940 & 3,4662 \\
\hline & & & & Média(20\%) & 14,2761 & 3,5937 & 5,1983 & 3,3109 \\
\hline & & & & $\operatorname{EM}(20 \%)$ & 13,1976 & 3,7865 & 5,1245 & 4,7647 \\
\hline & & & & $\mathrm{CP}(20 \%)$ & 15,3758 & 3,1965 & 5,1603 & 3,5843 \\
\hline \multirow{6}{*}{30} & \multirow{6}{*}{0,8} & \multirow{6}{*}{$\Sigma_{3}=$} & \multirow{6}{*}{$\left.\begin{array}{cc}20 & 16 \\
16 & 20\end{array}\right)$} & expressão & 4,1190 & 16,1102 & 1,2638 & 5,5974 \\
\hline & & & & boots completo & 3,9559 & 15,6904 & 1,1420 & 4,3776 \\
\hline & & & & BLUP(20\%) & 3,6908 & 14,7440 & 1,4236 & 4,2970 \\
\hline & & & & Média(20\%) & 4,1003 & 14,7859 & 1,4869 & 5,4432 \\
\hline & & & & $\operatorname{EM}(20 \%)$ & 3,5403 & 13,9392 & 1,6279 & 6,1241 \\
\hline & & & & $\mathrm{CP}(20 \%)$ & 3,7499 & 15,8511 & 1,3782 & 6,1333 \\
\hline
\end{tabular}


Tabela 4.3.6: Médias e desvios padrão das 2000 estimativas das componentes da variância na normal bivariada $(n=30, p=40 \%$ )

\begin{tabular}{|c|c|c|c|c|c|c|c|c|}
\hline$n$ & $\rho$ & Normal & & Método & $\overline{\hat{\sigma}}^{2}$ & $\hat{\sigma}_{b}^{2}$ & $\hat{S}$ & $\hat{S}_{b}$ \\
\hline \multirow{6}{*}{30} & \multirow{6}{*}{0,5} & \multirow{6}{*}{\multicolumn{2}{|c|}{$\boldsymbol{\Sigma}_{\mathbf{1}}=\left(\begin{array}{ll}8 & 4 \\
4 & 8\end{array}\right)$}} & expressão & 4,0099 & 3,8988 & 1,1565 & 1,5487 \\
\hline & & & & boots completo & 3,9244 & 3,7415 & 1,1087 & 1,2509 \\
\hline & & & & BLUP(40\%) & 3,7410 & 3,3107 & 1,6829 & 1,8929 \\
\hline & & & & Média(40\%) & 3,9201 & 3,7321 & 1,4825 & 1,7893 \\
\hline & & & & $\operatorname{EM}(40 \%)$ & 3,2265 & 3,8565 & 1,5447 & 1,9856 \\
\hline & & & & $\mathrm{CP}(40 \%)$ & 3,3789 & 3,7841 & 1,8954 & 1,6390 \\
\hline \multirow{6}{*}{30} & \multirow{6}{*}{0,2} & \multirow{6}{*}{$\Sigma_{2}=$} & \multirow{6}{*}{$\left(\begin{array}{cc}20 & 4 \\
4 & 20\end{array}\right)$} & expressão & 16,0078 & 4,1289 & 4,6547 & 3,1289 \\
\hline & & & & boots completo & 15,1267 & 4,3759 & 4,3467 & 3,0019 \\
\hline & & & & BLUP(40\%) & 13,8597 & 3,5798 & 5,2614 & 3,8148 \\
\hline & & & & Média(40\%) & 13,6738 & 3,7197 & 5,3757 & 3,5892 \\
\hline & & & & $\operatorname{EM}(40 \%)$ & 13,9735 & 3,6265 & 5,6334 & 3,9163 \\
\hline & & & & $\mathrm{CP}(40 \%)$ & 14,8749 & 3,7932 & 5,5652 & 3,4295 \\
\hline \multirow{6}{*}{30} & \multirow{6}{*}{0,8} & \multirow{6}{*}{$\Sigma_{3}=$} & \multirow{6}{*}{$\left.\begin{array}{cc}20 & 16 \\
16 & 20\end{array}\right)$} & expressão & 4,1190 & 16,1102 & 1,2638 & 5,5974 \\
\hline & & & & boots completo & 3,9559 & 15,6904 & 1,1420 & 4,3776 \\
\hline & & & & BLUP(40\%) & 3,5366 & 14,8739 & 1,8796 & 5,5071 \\
\hline & & & & Média(40\%) & 4,0043 & 14,3641 & 1,3889 & 5,4987 \\
\hline & & & & $\operatorname{EM}(40 \%)$ & 3,5474 & 14.2102 & 1,9857 & 5,5792 \\
\hline & & & & $\mathrm{CP}(40 \%)$ & 3,4847 & 14,6771 & 2,1075 & 5,5861 \\
\hline
\end{tabular}


Tabela 4.3.5a: Médias e desvios padrão das 2000 estimativas das variâncias das componentes da variância na normal bivariada $(n=30, p=20 \%)$

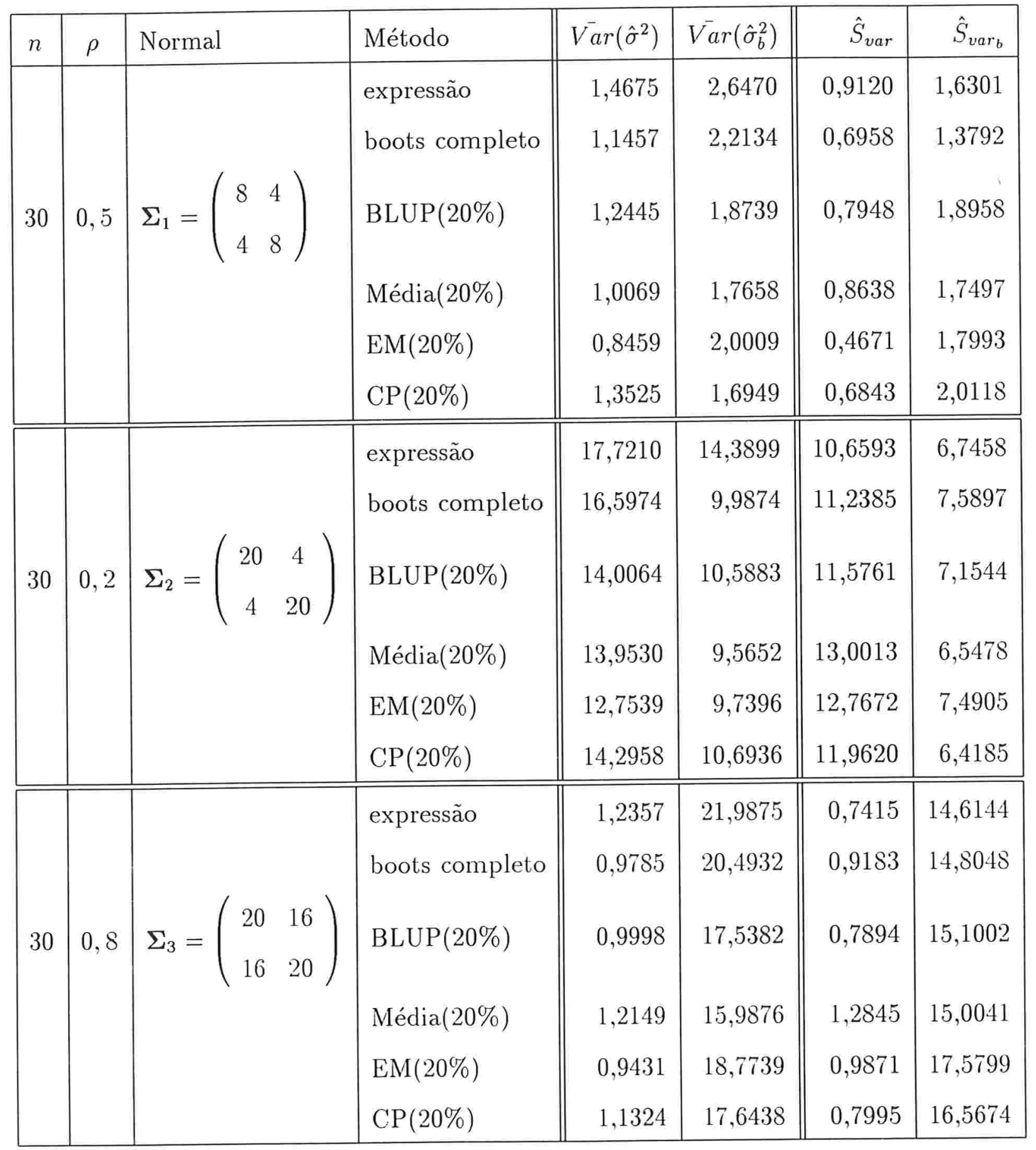


Tabela 4.3.6a: Médias e desvios padrão das 2000 estimativas das variâncias das componentes da variância na normal bivariada $(n=30, p=40 \%)$

\begin{tabular}{|c|c|c|c|c|c|c|c|c|}
\hline$n$ & $\rho$ & Normal & & Método & $\overline{\operatorname{Var}}\left(\hat{\sigma}^{2}\right)$ & $\operatorname{Var}\left(\hat{\sigma}_{b}^{2}\right)$ & $\hat{S}_{\text {var }}$ & $\hat{S}_{\text {var }_{b}}$ \\
\hline \multirow{6}{*}{30} & \multirow{6}{*}{0,5} & \multirow{6}{*}{\multicolumn{2}{|c|}{$\Sigma_{1}=\left(\begin{array}{ll}8 & 4 \\
4 & 8\end{array}\right)$}} & expressão & 1,4675 & 2,6470 & 0,9120 & 1,6301 \\
\hline & & & & boots completo & 1,1457 & 2,2134 & 0,6958 & 1,3792 \\
\hline & & & & BLUP(40\%) & 1,1198 & 1,6908 & 0,7124 & 1,5998 \\
\hline & & & & Média(40\%) & 0,7856 & 1,6123 & 0,7158 & 1,4562 \\
\hline & & & & $\operatorname{EM}(40 \%)$ & 0,6876 & 1,8549 & 0,4378 & 1,7356 \\
\hline & & & & $\mathrm{CP}(40 \%)$ & 1,2145 & 1,6759 & 0,4895 & 1,6436 \\
\hline \multirow{6}{*}{30} & \multirow{6}{*}{0,2} & \multirow{6}{*}{$\Sigma_{2}=$} & \multirow{6}{*}{$\left.\begin{array}{cc}20 & 4 \\
4 & 20\end{array}\right)$} & expressão & 17,7210 & 14,3899 & 10,6593 & 6,7458 \\
\hline & & & & boots completo & 16,5974 & 9,9874 & 11,2385 & 7,5897 \\
\hline & & & & BLUP(40\%) & 12,6784 & 9,4538 & 11,1863 & 6,9886 \\
\hline & & & & Média(40\%) & 12,8759 & 9,8367 & 11,7495 & 5,9947 \\
\hline & & & & $\operatorname{EM}(40 \%)$ & 12,0056 & 9,0013 & 12,2768 & 6,4127 \\
\hline & & & & $\mathrm{CP}(40 \%)$ & 13,5649 & 8,2643 & 10,4763 & 6,8359 \\
\hline \multirow{6}{*}{30} & \multirow{6}{*}{0,8} & \multirow{6}{*}{$\Sigma_{3}=$} & \multirow{6}{*}{$\left.\begin{array}{ll}20 & 16 \\
16 & 20\end{array}\right)$} & expressão & 1,2357 & 21,9875 & 0,7415 & 14,6144 \\
\hline & & & & boots completo & 0,9785 & 20,4932 & 0,9183 & 14,8048 \\
\hline & & & & BLUP $(40 \%)$ & 0,9546 & 16,1836 & 0,6375 & 15,7126 \\
\hline & & & & Média(40\%) & 1,1004 & 15,5627 & 1,1429 & 14,0115 \\
\hline & & & & $\operatorname{EM}(40 \%)$ & 0,8471 & 17,9004 & 0,8126 & 16,6548 \\
\hline & & & & $\mathrm{CP}(40 \%)$ & 1,1386 & 15,3932 & 0,9345 & 14,3005 \\
\hline
\end{tabular}


Tabela 4.3.7: Médias e desvios padrão das 2000 estimativas das componentes da variância na normal bivariada $(n=100, p=20 \%)$

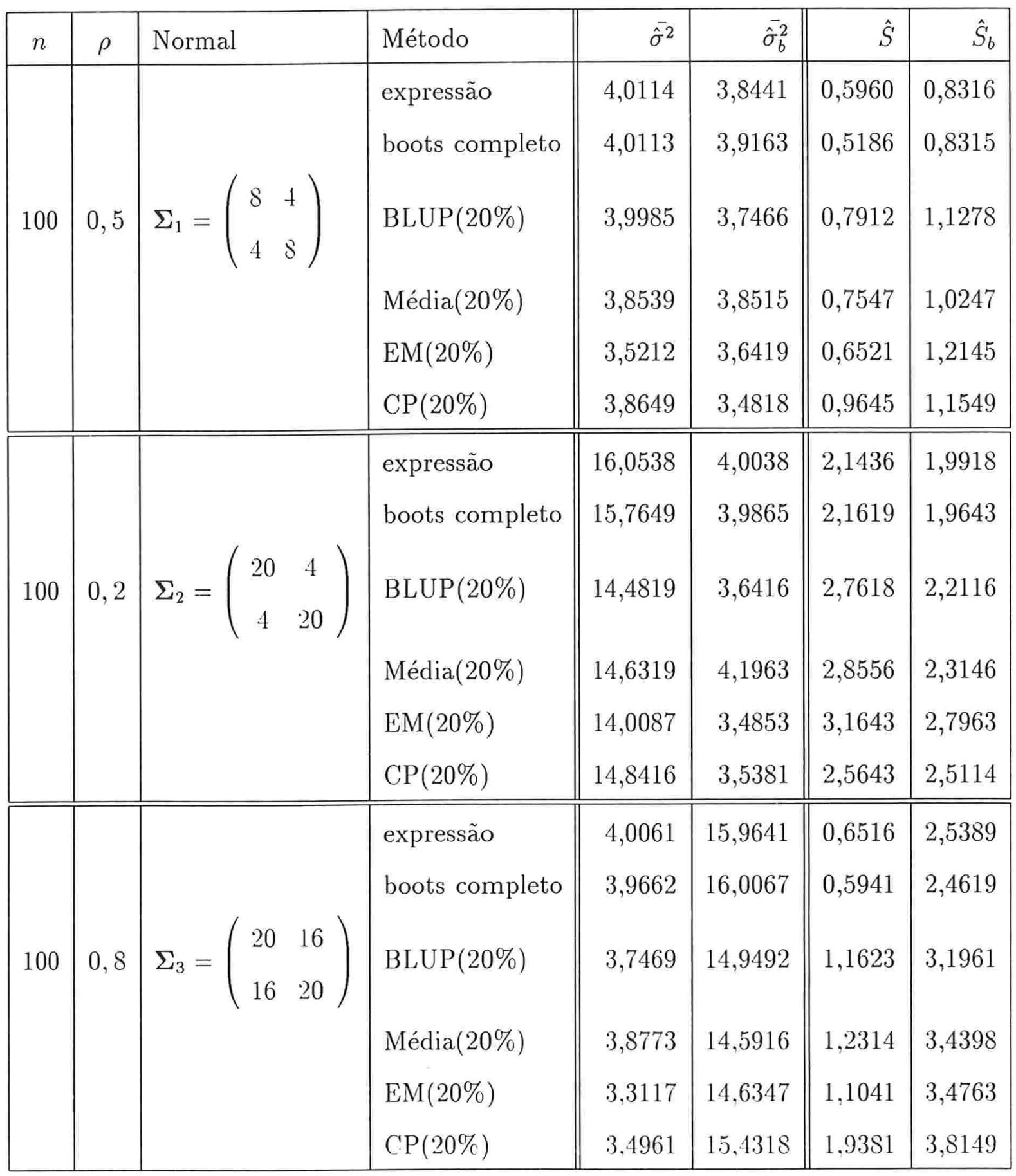


Tabela 4.3.8: Médias e desvios padrão das 2000 estimativas das componentes da variância na normal bivariada $(n=100, p=40 \%)$

\begin{tabular}{|c|c|c|c|c|c|c|c|c|}
\hline$n$ & $\rho$ & Normal & & Método & $\hat{\sigma}^{2}$ & $\hat{\sigma}_{b}^{2}$ & $\hat{S}$ & $\hat{S}_{b}$ \\
\hline \multirow{6}{*}{100} & \multirow{6}{*}{0,5} & \multirow{6}{*}{$\Sigma_{1}=$} & \multirow{6}{*}{$\left.\begin{array}{ll}8 & 4 \\
4 & 8\end{array}\right)$} & expressão & 4,0114 & 3,8441 & 0,5960 & 0,8316 \\
\hline & & & & boots completo & 4,0113 & 3,9163 & 0,5186 & 0,8315 \\
\hline & & & & BLUP(40\%) & 3,8643 & 3,7654 & 0,8256 & 1,1832 \\
\hline & & & & Média(40\%) & 3,9341 & 3,7361 & 0,8943 & 1,1263 \\
\hline & & & & $\operatorname{EM}(40 \%)$ & 3,4116 & 3,6341 & 0,7943 & 1,2943 \\
\hline & & & & $\mathrm{CP}(40 \%)$ & 3,7143 & 3,8616 & 1,0463 & 1,1858 \\
\hline \multirow{6}{*}{100} & \multirow{6}{*}{0,2} & \multirow{6}{*}{$\Sigma_{2}=$} & \multirow{6}{*}{$\left.\begin{array}{cc}20 & 4 \\
4 & 20\end{array}\right)$} & expressão & 16,0538 & 4,0038 & 2,1436 & 1,9918 \\
\hline & & & & boots completo & 15,7649 & 3,9865 & 2,1619 & 1,9643 \\
\hline & & & & BLUP(40\%) & 14,4819 & 3,6416 & 2,7618 & 2,2116 \\
\hline & & & & Média(40\%) & 14,5160 & 3,8318 & 3,1614 & 2,6389 \\
\hline & & & & $\operatorname{EM}(40 \%)$ & 13,9381 & 3,1618 & 3,2163 & 2,9147 \\
\hline & & & & $\mathrm{CP}(40 \%)$ & 14,6345 & 3,2618 & 3,1841 & 2,6143 \\
\hline \multirow{6}{*}{100} & \multirow{6}{*}{0,8} & \multirow{6}{*}{$\Sigma_{3}=$} & \multirow{6}{*}{$\left.\begin{array}{cc}20 & 16 \\
16 & 20\end{array}\right)$} & expressão & 4,0061 & 15,9641 & 0,6516 & 2,5389 \\
\hline & & & & boots completo & 3,9662 & 16,0067 & 0,5941 & 2,4619 \\
\hline & & & & BLUP(40\%) & 3,5154 & 14,4921 & 1,2852 & 3,4676 \\
\hline & & & & Média(40\%) & 3,6843 & 14,5815 & 1,3983 & 3,8805 \\
\hline & & & & $\operatorname{EM}(40 \%)$ & 3,2181 & 14.4401 & 1,1470 & 3,8695 \\
\hline & & & & $\mathrm{CP}(40 \%)$ & 3,6304 & 15.4116 & 1.9683 & 3,9863 \\
\hline
\end{tabular}


Tabela 4.3.7a: Médias e desvios padrão das 2000 estimativas das variâncias das componentes da variância na normal bivariada $(n=100, p=20 \%$ )

\begin{tabular}{|c|c|c|c|c|c|c|c|c|}
\hline$n$ & $\rho$ & Normal & & Método & $\overline{\operatorname{Var}}\left(\hat{\sigma}^{2}\right)$ & $\overline{\operatorname{Var}}\left(\hat{\sigma}_{b}^{2}\right)$ & $\hat{S}_{v a r}$ & $\hat{S}_{\text {var }_{b}}$ \\
\hline \multirow{6}{*}{100} & \multirow{6}{*}{0,5} & \multirow{6}{*}{$\Sigma_{1}=$} & \multirow{6}{*}{$\left(\begin{array}{ll}8 & 4 \\
4 & 8\end{array}\right)$} & expressão & 0,3946 & 0,8154 & 0,1004 & 0,1946 \\
\hline & & & & boots completo & 0,3619 & 0,7513 & 0,0986 & 0,2319 \\
\hline & & & & BLUP(20\%) & 0,3314 & 0,6942 & 0,1223 & 0,3516 \\
\hline & & & & Média(20\%) & 0,3219 & 0,6318 & 0,1718 & 0,3468 \\
\hline & & & & $\operatorname{EM}(20 \%)$ & 0,2875 & 0,5946 & 0,1129 & 0,3016 \\
\hline & & & & $\mathrm{CP}(20 \%)$ & 0,5019 & 0,6765 & 0,3245 & 0,3871 \\
\hline \multirow{6}{*}{100} & \multirow{6}{*}{0,2} & \multirow{6}{*}{$\Sigma_{2}=$} & \multirow{6}{*}{$\left.\begin{array}{cc}20 & 4 \\
4 & 20\end{array}\right)$} & expressão & 5,0068 & 4,2688 & 1,5649 & 0,9218 \\
\hline & & & & boots completo & 4,8619 & 3,7133 & 1,9512 & 1,3996 \\
\hline & & & & BLUP(20\%) & 4,1846 & 2,6641 & 2,3164 & 1,6014 \\
\hline & & & & Média(20\%) & 4,2754 & 2,6518 & 2,3173 & 1,6098 \\
\hline & & & & $\operatorname{EM}(20 \%)$ & 4,1865 & 2,7218 & 2,3100 & 1,6345 \\
\hline & & & & $\mathrm{CP}(20 \%)$ & 4,8098 & 2,7635 & 2,8419 & 1,8114 \\
\hline \multirow{6}{*}{100} & \multirow{6}{*}{0,8} & \multirow{6}{*}{$\Sigma_{3}=$} & \multirow{6}{*}{$\left.\begin{array}{cc}20 & 16 \\
16 & 20\end{array}\right)$} & expressão & 0,3163 & 6,5318 & 0,1003 & 1,7614 \\
\hline & & & & boots completo & 0,2918 & 6,3512 & 0,1256 & 2,5014 \\
\hline & & & & BLUP(20\%) & 1,3948 & 5,8347 & 1,2814 & 3,1819 \\
\hline & & & & Média(20\%) & 1,6379 & 5,2546 & 0,4963 & 3,1452 \\
\hline & & & & $\operatorname{EM}(20 \%)$ & 1,2669 & 5,9019 & 0,3188 & 3,2848 \\
\hline & & & & $\mathrm{CP}(20 \%)$ & 1,4157 & 5,7695 & 1,5612 & 3,3219 \\
\hline
\end{tabular}


Tabela 4.3.8a: Médias e desvios padrão das 2000 estimativas das variâncias das componentes da variância na normal bivariada ( $n=100, p=40 \%$ )

\begin{tabular}{|c|c|c|c|c|c|c|c|c|}
\hline$n$ & $\rho$ & Normal & & Método & $\overline{\operatorname{Var}}\left(\hat{\sigma}^{2}\right)$ & $\operatorname{Var}\left(\hat{\sigma}_{b}^{2}\right)$ & $\hat{S}_{\text {var }}$ & $\hat{S}_{\text {varb }_{b}}$ \\
\hline \multirow{6}{*}{100} & \multirow{6}{*}{0,5} & \multirow{6}{*}{\multicolumn{2}{|c|}{$\Sigma_{1}=\left(\begin{array}{ll}0 & 4 \\
4 & 8\end{array}\right)$}} & expressão & 0,3946 & 0,8154 & 0,1004 & 0,1946 \\
\hline & & & & boots completo & 0,3619 & 0,7513 & 0,0986 & 0,2319 \\
\hline & & & & BLUP $(40 \%)$ & 0,3318 & 0,6318 & 0,1631 & 0,3218 \\
\hline & & & & Média(40\%) & 0,3309 & 0,6178 & 0,1563 & 0,3391 \\
\hline & & & & $\operatorname{EM}(40 \%)$ & 0,2218 & 0,4282 & 0,1214 & 0,2843 \\
\hline & & & & $\mathrm{CP}(40 \%)$ & 0,3618 & 0,6126 & 0,1843 & 0,3143 \\
\hline \multirow{6}{*}{100} & \multirow{6}{*}{0,2} & \multirow{6}{*}{$\Sigma_{2}=$} & \multirow{6}{*}{$\left.\begin{array}{cc}20 & 4 \\
4 & 20\end{array}\right)$} & expressão & 5,0068 & 4,2688 & 1,5649 & 0,9218 \\
\hline & & & & boots completo & 4,8619 & 3,7133 & 1,9512 & 1,3996 \\
\hline & & & & BLUP(40\%) & 4,3267 & 2,6198 & 2,5247 & 1,8001 \\
\hline & & & & Média(40\%) & 4,5478 & 2,5439 & 2,6064 & 1,6538 \\
\hline & & & & $\operatorname{EM}(40 \%)$ & 4,6728 & 2,1649 & 2,4861 & 1,5438 \\
\hline & & & & $\mathrm{CP}(40 \%)$ & 4,5436 & 2,3948 & 2,3156 & 1,4176 \\
\hline \multirow{6}{*}{100} & \multirow{6}{*}{0,8} & \multirow{6}{*}{\multicolumn{2}{|c|}{$\Sigma_{3}=\left(\begin{array}{ll}20 & 16 \\
16 & 20\end{array}\right)$}} & expressão & 0,3163 & 6,5318 & 0,1003 & 1,7614 \\
\hline & & & & boots completo & 0,2918 & 6,3512 & 0,1256 & 2,5014 \\
\hline & & & & BLUP $(40 \%)$ & 1,2351 & 5,6942 & 1,1926 & 3,6381 \\
\hline & & & & Média(40\%) & 1,2639 & 5,4620 & 0,9854 & 3,4261 \\
\hline & & & & $\operatorname{EM}(40 \%)$ & 1,1728 & 5,6395 & 1,0038 & 3,4263 \\
\hline & & & & $\mathrm{CP}(40 \%)$ & 1,2571 & 5,3960 & 1,0236 & 3,4372 \\
\hline
\end{tabular}


Tabela 4.3.9: Coeficientes de variação das componentes da variância no modelo linear misto

\begin{tabular}{|c|c|c|c|c|c|c|}
\hline \multirow{2}{*}{ Método } & \multirow[b]{2}{*}{$n$} & \multirow[b]{2}{*}{$\rho$} & \multicolumn{2}{|l|}{$p=20 \%$} & \multicolumn{2}{|l|}{$p=40 \%$} \\
\hline & & & $\operatorname{cv}\left(\hat{\sigma}^{2}\right)$ & $c v\left(\hat{\sigma}_{b}^{2}\right)$ & $\operatorname{cv}\left(\hat{\sigma}^{2}\right)$ & $c v\left(\hat{\sigma}_{b}^{2}\right)$ \\
\hline \multirow{6}{*}{ BLUP } & \multirow{3}{*}{30} & 0,5 & 0,31 & 0,43 & 0,43 & 0,61 \\
\hline & & 0,2 & 0,35 & 0,90 & 0,38 & 1,06 \\
\hline & & 0,8 & 0,45 & 0,30 & 0,56 & 0,34 \\
\hline & \multirow{3}{*}{100} & 0,5 & 0,19 & 0,30 & 0,22 & 0,31 \\
\hline & & 0,2 & 0,19 & 0,61 & 0,20 & 0,62 \\
\hline & & 0,8 & 0,31 & 0,21 & 0,36 & 0,24 \\
\hline \multirow{6}{*}{ Média } & \multirow{3}{*}{30} & 0,5 & 0,37 & 0,47 & 0,43 & 0,52 \\
\hline & & 0,2 & 0,36 & 0,89 & 0,38 & 0,93 \\
\hline & & 0,8 & 0,36 & 0,35 & 0,40 & 0,38 \\
\hline & \multirow{3}{*}{100} & 0,5 & 0,19 & 0,28 & 0,22 & 0,31 \\
\hline & & 0,2 & 0,19 & 0,59 & 0,22 & 0,69 \\
\hline & & 0,8 & 0,37 & 0,25 & 0,37 & 0,26 \\
\hline \multirow{6}{*}{ EM } & \multirow{3}{*}{30} & 0,5 & 0,42 & 0,51 & 0,61 & 0,45 \\
\hline & & 0,2 & 0,41 & 1,18 & 0,40 & 1,04 \\
\hline & & 0,8 & 0,36 & 0,43 & 0,61 & 0,41 \\
\hline & \multirow{3}{*}{100} & 0,5 & 0,23 & 0,38 & 0,24 & 0,39 \\
\hline & & 0,2 & 0,22 & 0,81 & 0,23 & 0,93 \\
\hline & & 0,8 & 0,30 & 0,23 & 0,35 & 0,27 \\
\hline \multirow{6}{*}{$\mathrm{CP}$} & \multirow{3}{*}{30} & 0,5 & 0,42 & 0,49 & 0,48 & 0,46 \\
\hline & & 0,2 & 0,35 & 1,15 & 0,39 & 0,94 \\
\hline & & 0,8 & 0,33 & 0,39 & 0,58 & 0,37 \\
\hline & \multirow{3}{*}{100} & 0,5 & 0,26 & 0,31 & 0,26 & 0,34 \\
\hline & & 0,2 & 0,17 & 0,72 & 0,22 & 0,80 \\
\hline & & 0,8 & 0,55 & 0,25 & 0,51 & 0,26 \\
\hline
\end{tabular}




\section{Conclusões}

O objetivo deste trabalho foi aplicar o bootstrap a dados incompletos. Usamos a técnica bootstrap primeiro para dados completos, tanto para dados gerados sob o modelo linear misto como a partir da distribuição normal bivariada. Observamos que, para as duas formas, os resultados foram similares.

No caso de dados completos, as estimativas foram calculadas pelas expressões dos estimadores e pelo bootstrap. Os resultados foram similares.

Aplicamos também o bootstrap a dados incompletos, conjuntamente com quatro métodos de imputação determinísticos, o BLUP, sustituição da média, algoritmo EM e componentes principais de Dear. Como já era esperado o fato de ter ausência de dados faz com que as estimativas das componentes da variância e as variâncias destas sejam subestimadas. Os resultados obtidos pelo BLUP e pelo método da média são similares e melhores do que os obtidos pelo algoritmo EM e o método de componentes principais.

Quando a razão entre as componentes da variância (variância do efeito sobre variância dos erros) é pequena, o erro padrão da variância do estimador da variância do efeito é grande, para amostras pequenas.

É claro que muito ainda pode ser feito. Algumas sugestões de tópicos para a continuidade deste trabalho são as seguintes:

1. considerar o bootstrap aplicado a dados incompletos usando reamostragem sem reposição (Sitter 1992); 
2. considerar o caso em que a amostra é selecionada de uma população finita;

3. aplicar métodos de imputação aleatórios, em especial a imputação múltipla;

4. fazer comparações com outros métodos de reamostragem como jackknife e validação cruzada;

5. considerar planejamentos amostrais mais complexos, como por exemplo amostra estratificada;

6. estudar a aplicação do bootstrap paramétrico e do bootstrap Bayesiano a dados incompletos. 


\section{Apêndice}

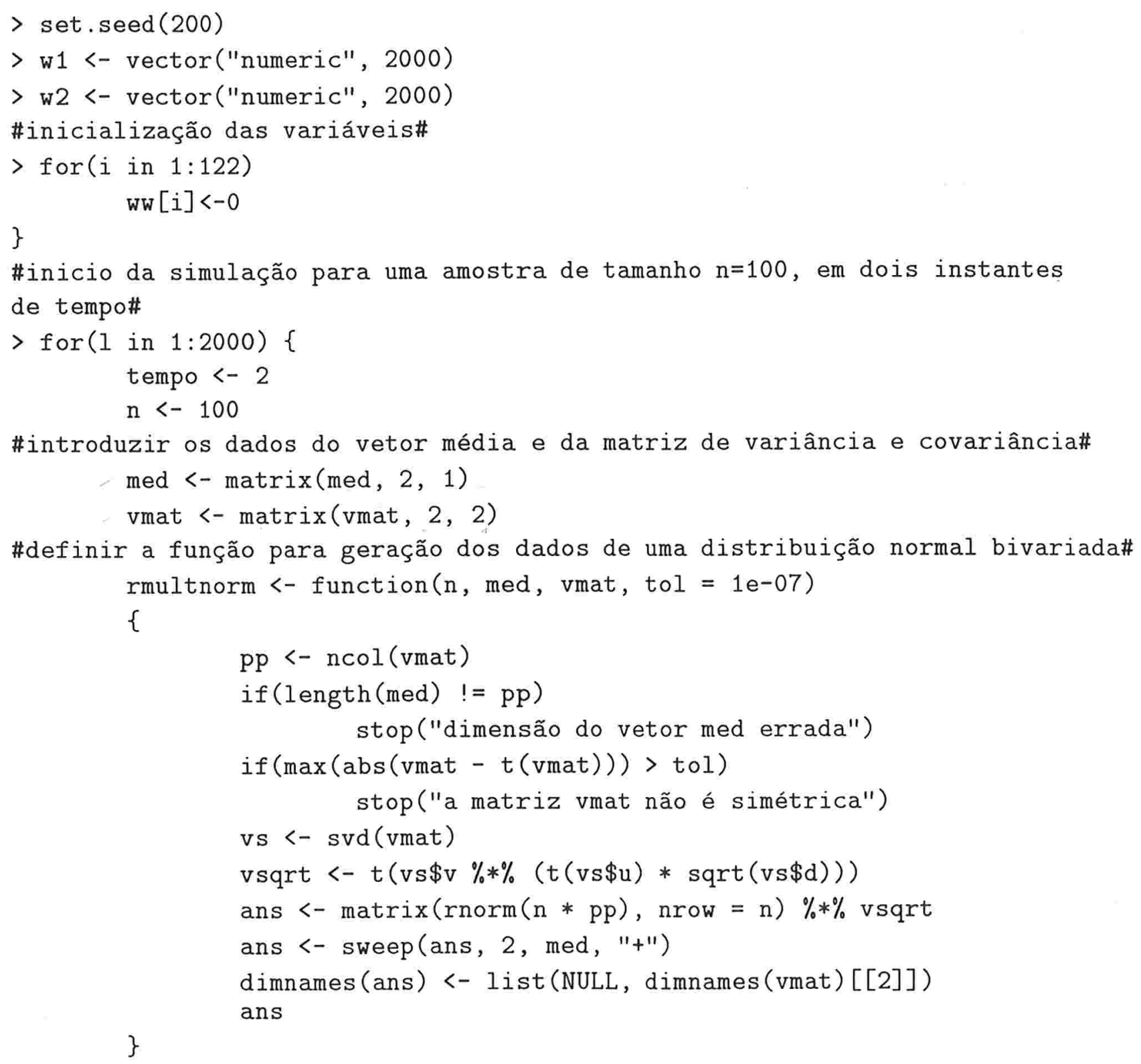


\#amostra gerada através da normal bivariada (y1; y2)\#

$$
\begin{aligned}
& \text { del <- rmultnorm(n, med, vmat) } \\
& \text { del <- } \operatorname{matrix}(\operatorname{del}, \mathrm{n}, 2) \\
& \mathrm{y} 1<-\operatorname{del}[1: \mathrm{n}, 1] \\
& \text { y2 <- del[1:n, 2] }
\end{aligned}
$$

\#cálculo das componentes da variância e de suas variâncias com os dados completos\#

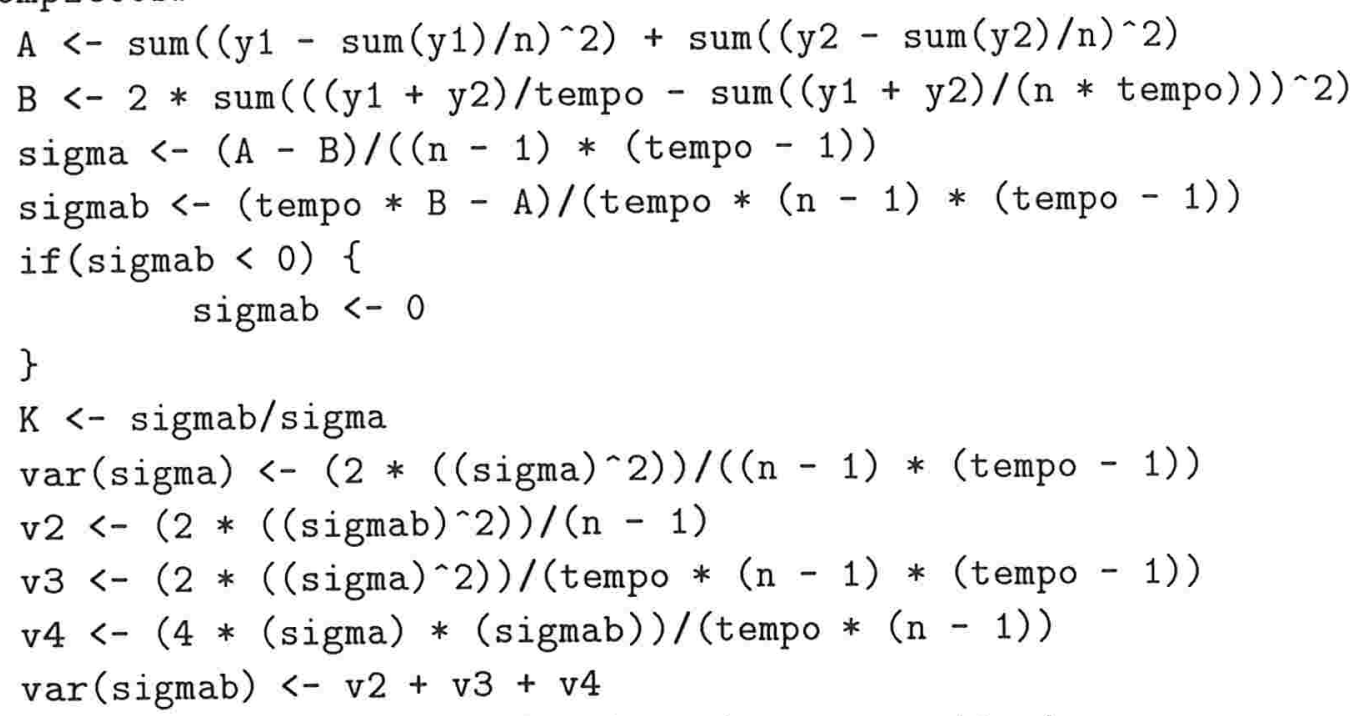




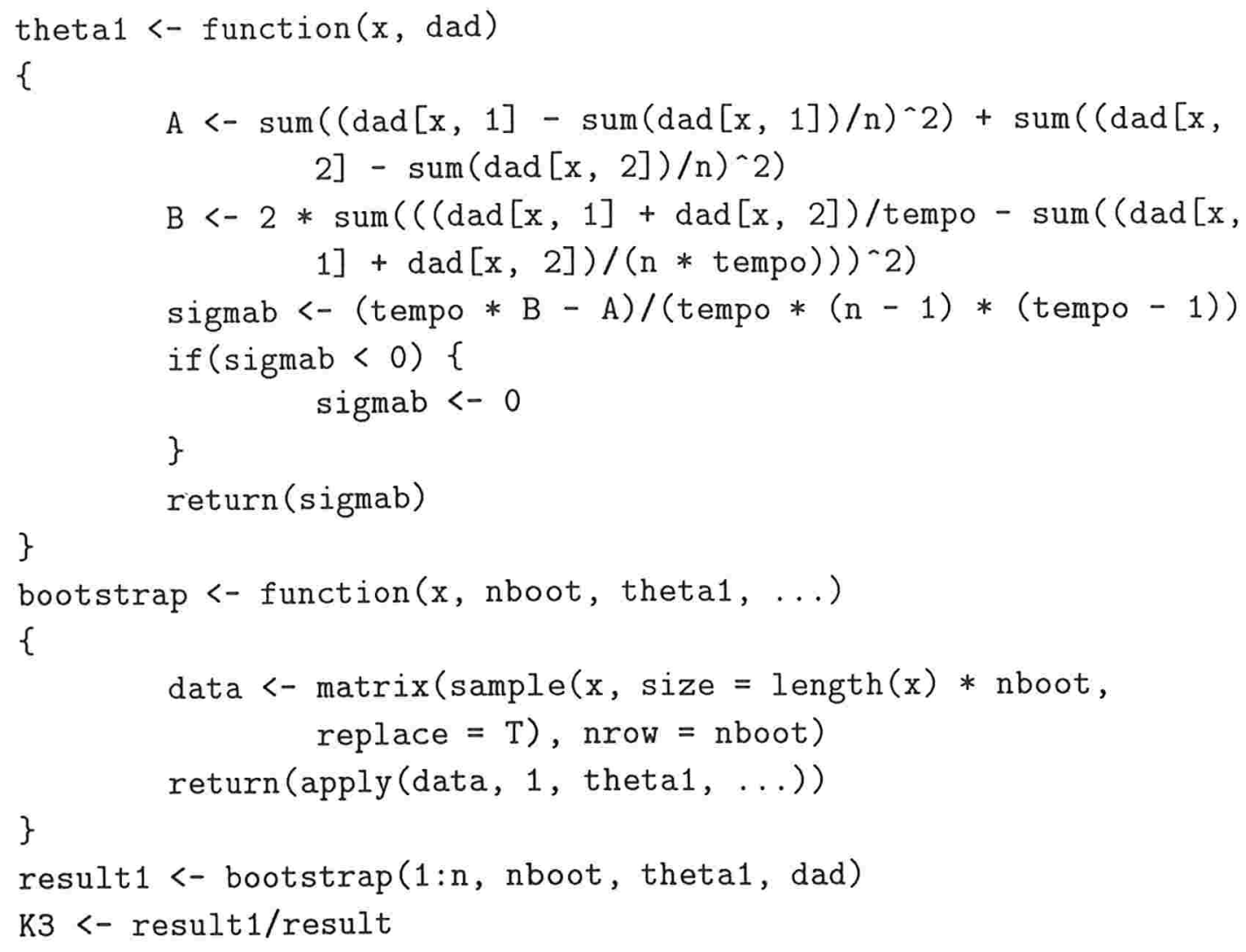




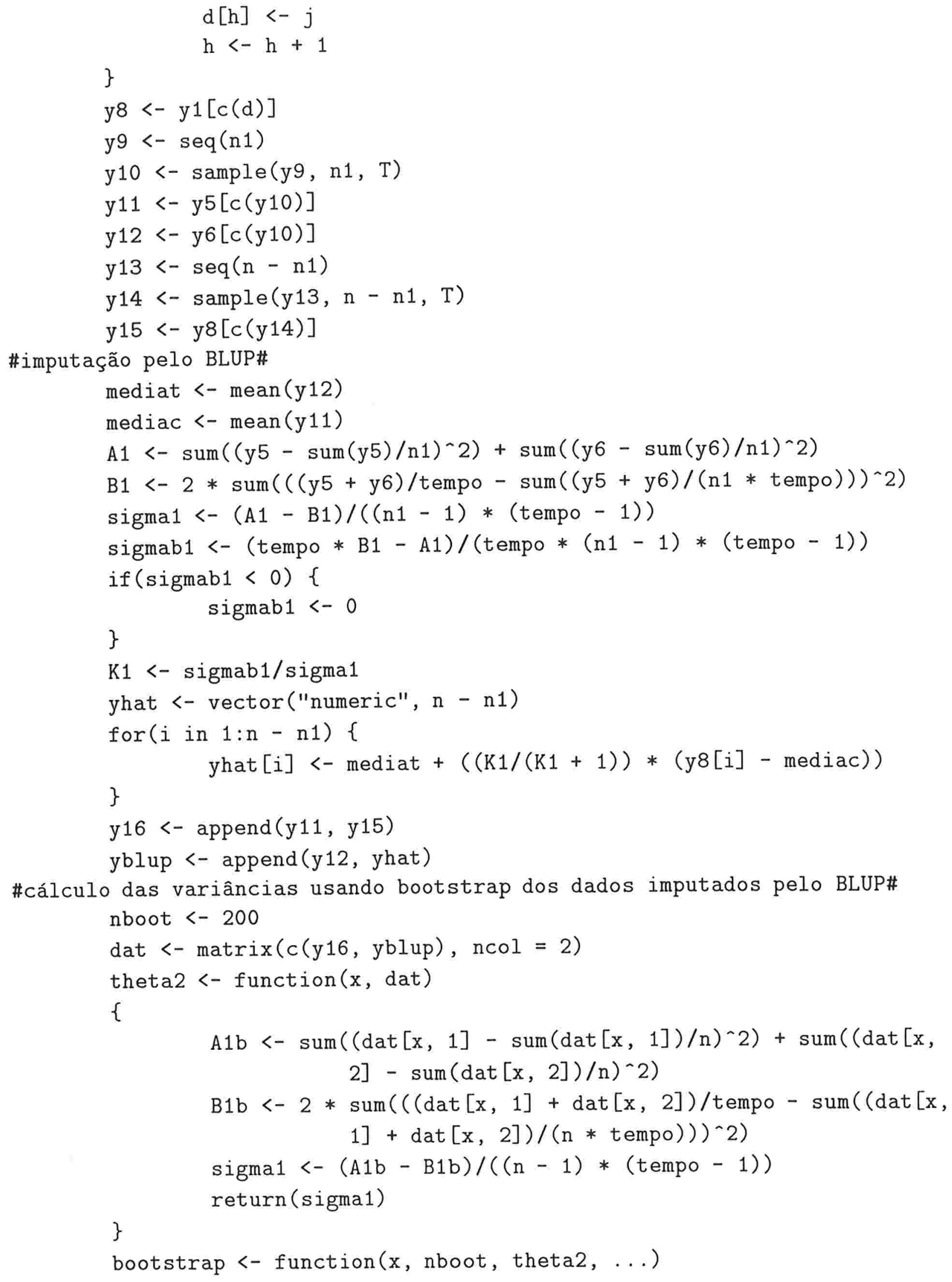


\{

data <- matrix $(\operatorname{sample}(x, \operatorname{size}=\operatorname{length}(x) *$ nboot, replace $=T)$, nrow $=$ nboot $)$

return (apply (data, 1, theta2, ...))

\}

$\operatorname{sig} 20<-$ bootstrap $(1: n$, nboot, theta2, dat $)$

theta3 <- function( $x$, dat)

\{

$\mathrm{A} 1 \mathrm{~b}<-\operatorname{sum}\left((\operatorname{dat}[\mathrm{x}, 1]-\operatorname{sum}(\operatorname{dat}[\mathrm{x}, 1]) / \mathrm{n})^{\wedge} 2\right)+\operatorname{sum}((\operatorname{dat}[\mathrm{x}$, 2] $\left.-\operatorname{sum}(\operatorname{dat}[x, 2]) / n)^{\wedge} 2\right)$

$\mathrm{B} 1 \mathrm{~b}<-2 * \operatorname{sum}(((\operatorname{dat}[\mathrm{x}, 1]+\operatorname{dat}[\mathrm{x}, 2]) /$ tempo $-\operatorname{sum}((\operatorname{dat}[\mathrm{x}$, 1] $+\operatorname{dat}[\mathrm{x}, 2]) /(\mathrm{n} *$ tempo $\left.)))^{\wedge} 2\right)$

sigmab1 <- $($ tempo*B1b - A1b)/(tempo * $(n-1) *($ tempo-1) $)$

if $($ sigmab1 $<0)\{$

sigmab1 $<-0$

\}

$\operatorname{return}($ sigmab1)

\}

bootstrap <- function(x, nboot, theta3,...)

\{

data <- matrix $(\operatorname{sample}(x, \operatorname{size}=\operatorname{length}(x) *$ nboot, replace $=T$ ), nrow $=$ nboot $)$

return (apply(data, 1, theta3, ...))

\}

$\operatorname{sigb20}<-$ bootstrap $(1: \mathrm{n}$, nboot, theta3, dat $)$

$\mathrm{K} 4 \mathrm{~b}<-$ sigb20/sig20

\#imputação pela média\#

y17<- vector("numeric", n - n1)

for ( $i$ in $1: n-n 1)$ \{

$\mathrm{y} 17[\mathrm{i}]<-\operatorname{mean}(\mathrm{y} 12)$

\}

ymedia <- append (y12, y17)

\#cálculo das variâncias usando bootstrap dos dados imputados pela média\#

datm <- matrix $(c(y 16$, ymedia $)$, ncol $=2)$

theta $4<-$ function $(x$, datm $)$

\{

$$
\begin{gathered}
\operatorname{A1m}<-\operatorname{sum}\left((\operatorname{datm}[\mathrm{x}, 1]-\operatorname{sum}(\operatorname{datm}[\mathrm{x}, 1]) / \mathrm{n})^{\wedge} 2\right)+\operatorname{sum}( \\
\left.\quad(\operatorname{datm}[\mathrm{x}, 2]-\operatorname{sum}(\operatorname{datm}[\mathrm{x}, 2]) / \mathrm{n})^{-} 2\right) \\
\mathrm{B} 1 \mathrm{~m}<-2 * \operatorname{sum}(((\operatorname{datm}[\mathrm{x}, 1]+\operatorname{datm}[\mathrm{x}, 2]) / \operatorname{tempo}-\operatorname{sum}( \\
\left.\quad(\operatorname{datm}[\mathrm{x}, 1]+\operatorname{datm}[\mathrm{x}, 2]) /(\mathrm{n} * \operatorname{tempo})))^{-} 2\right)
\end{gathered}
$$




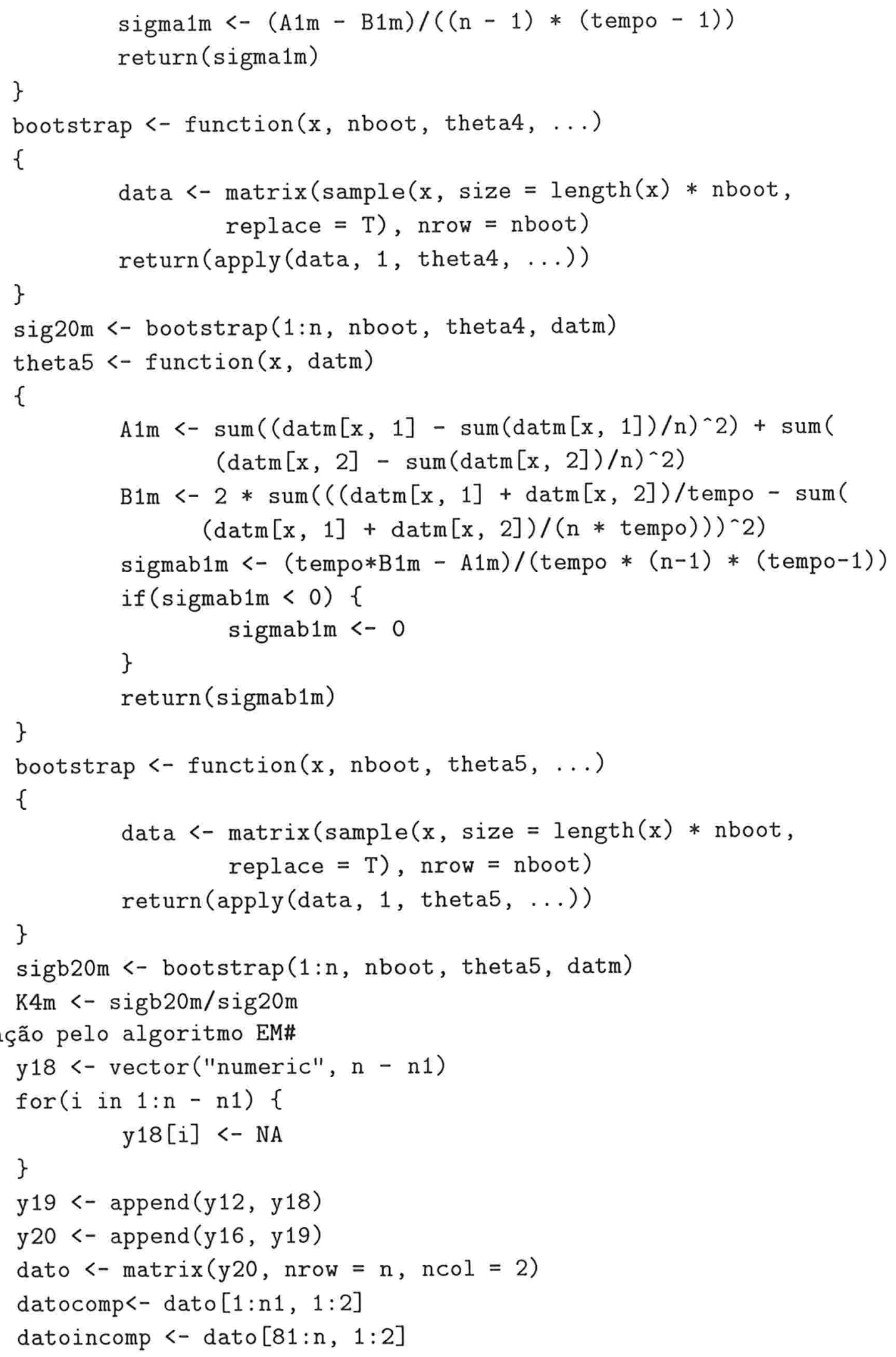




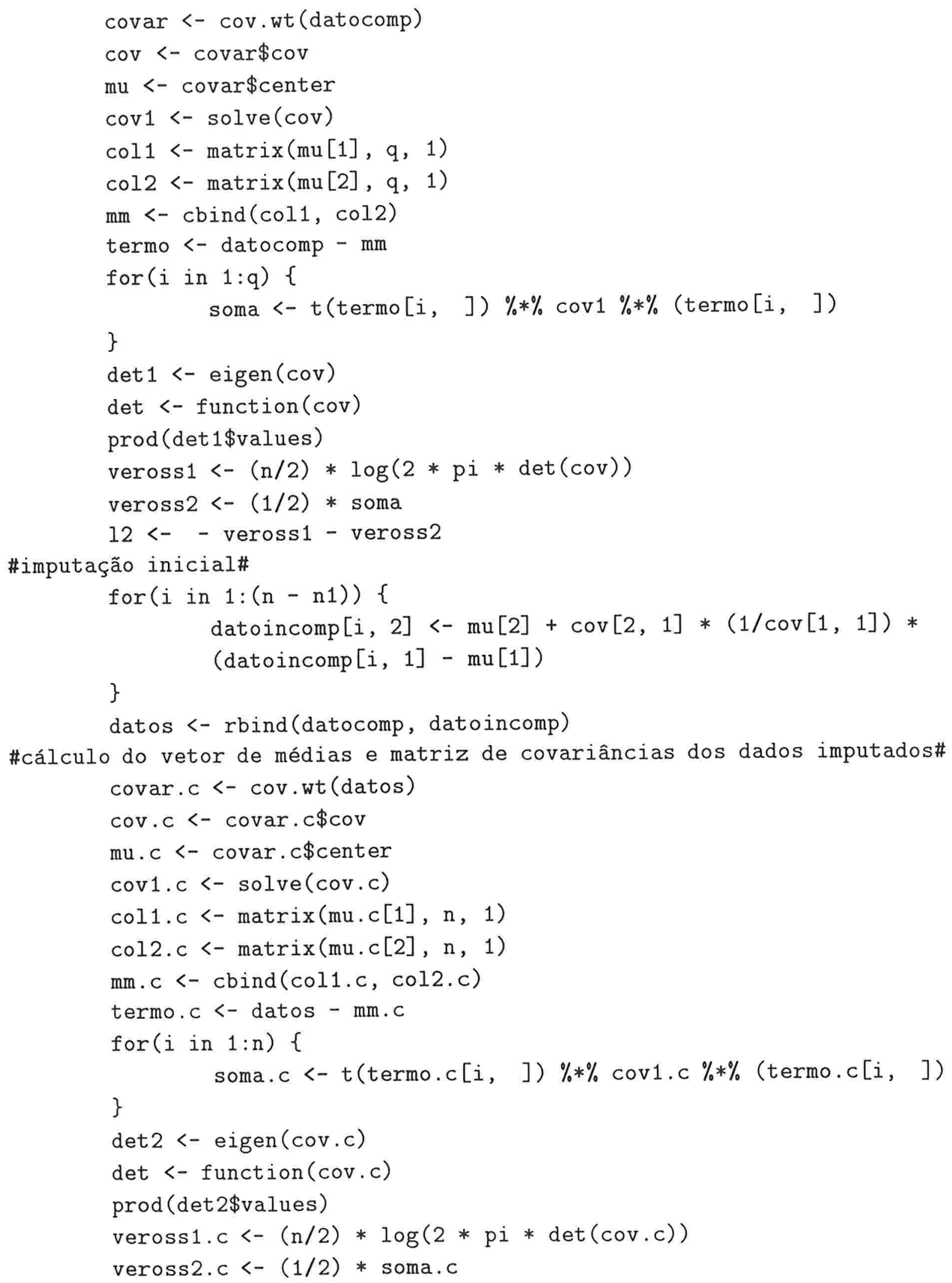


\#cálculo das variâncias usando bootstrap dos dados imputados pelo algoritmo EM\#

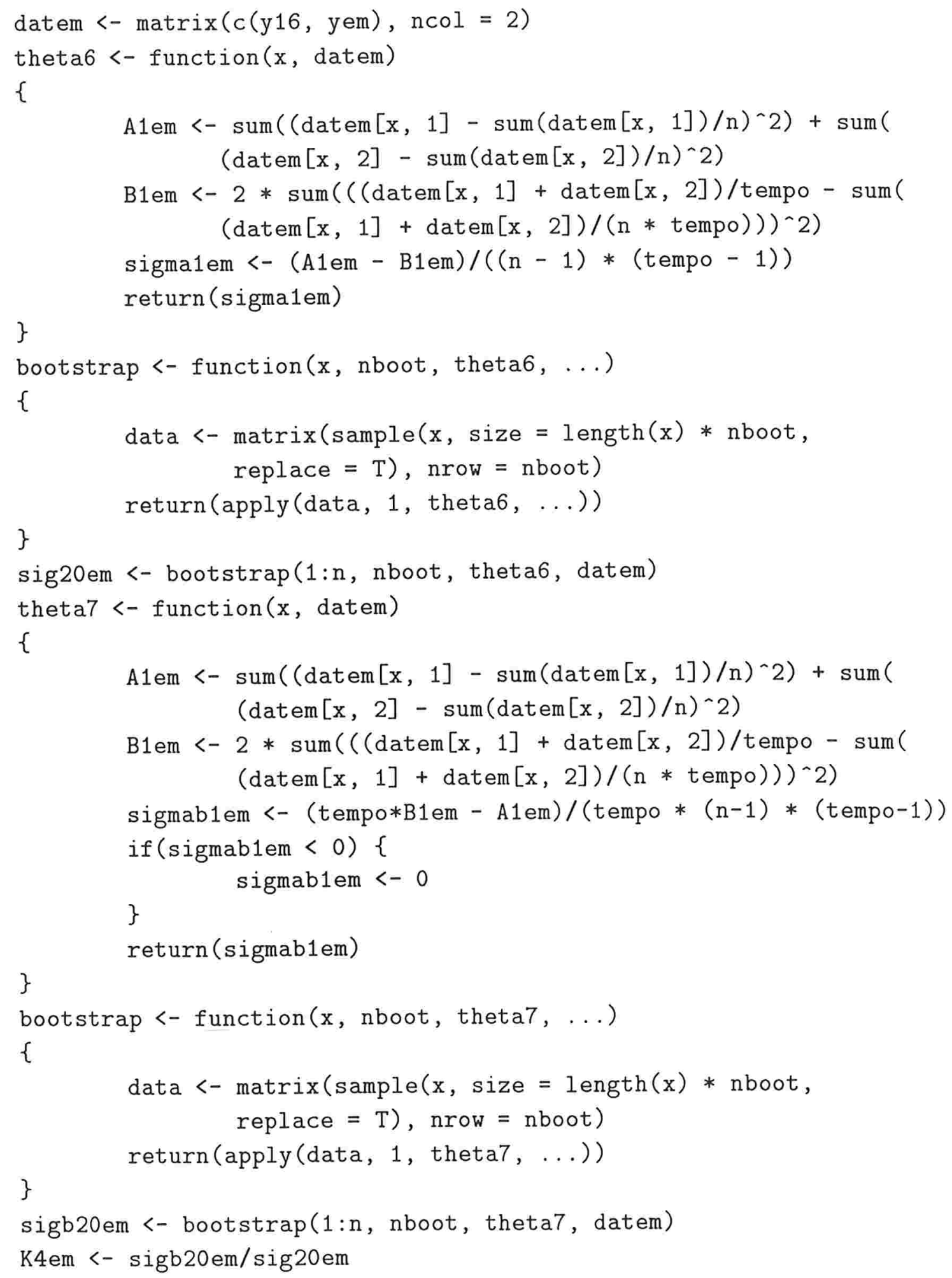




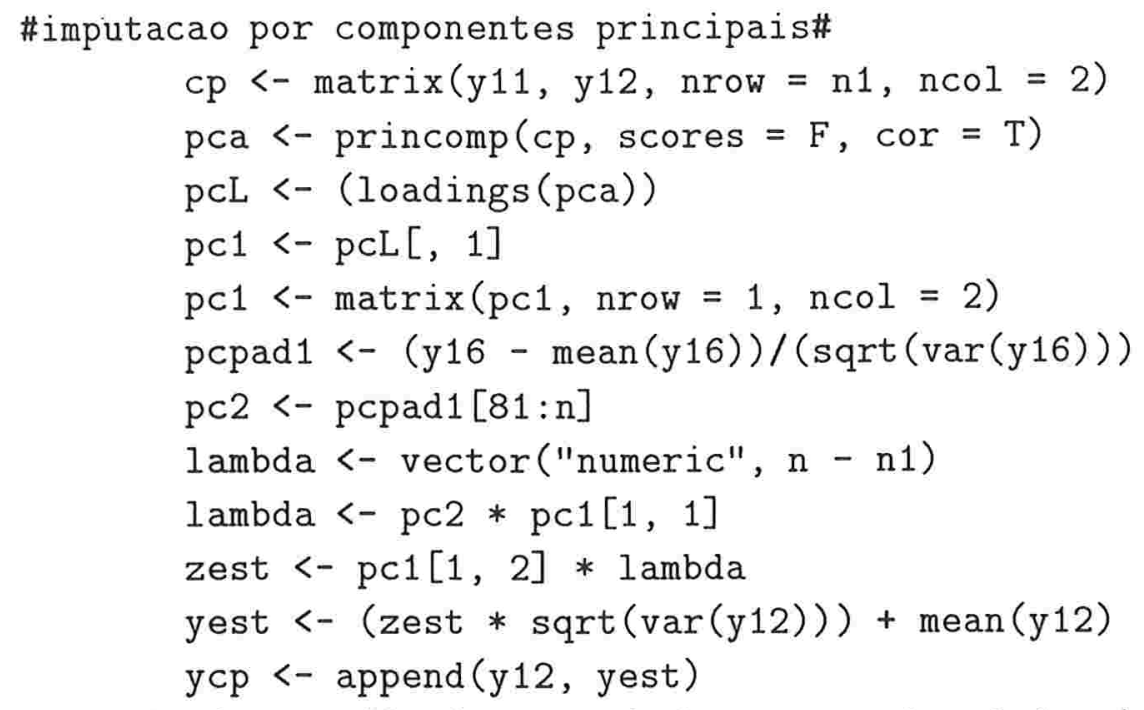

\#cálculo das variâncias usando bootstrap dos dados imputados por componentes principais\#

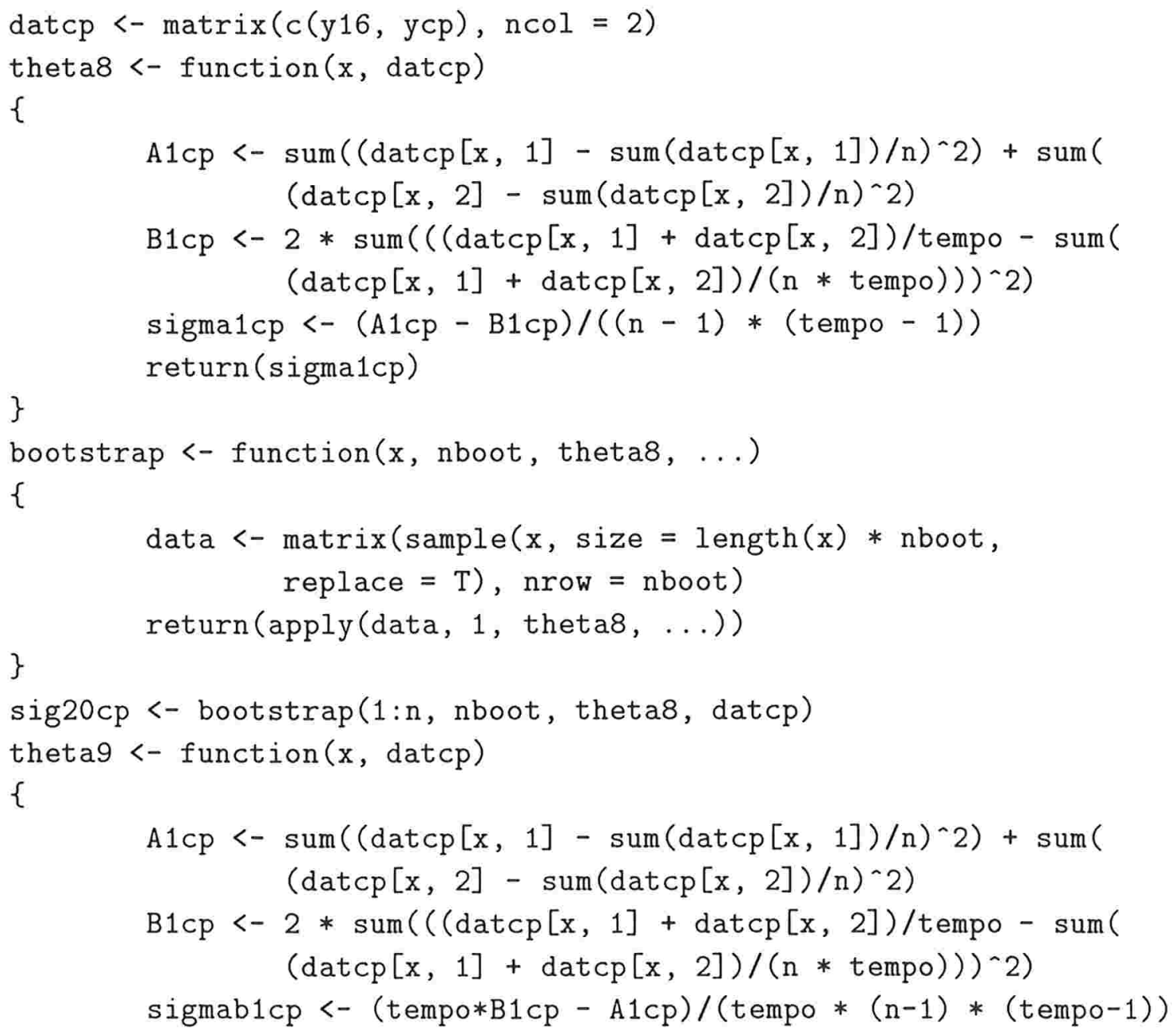




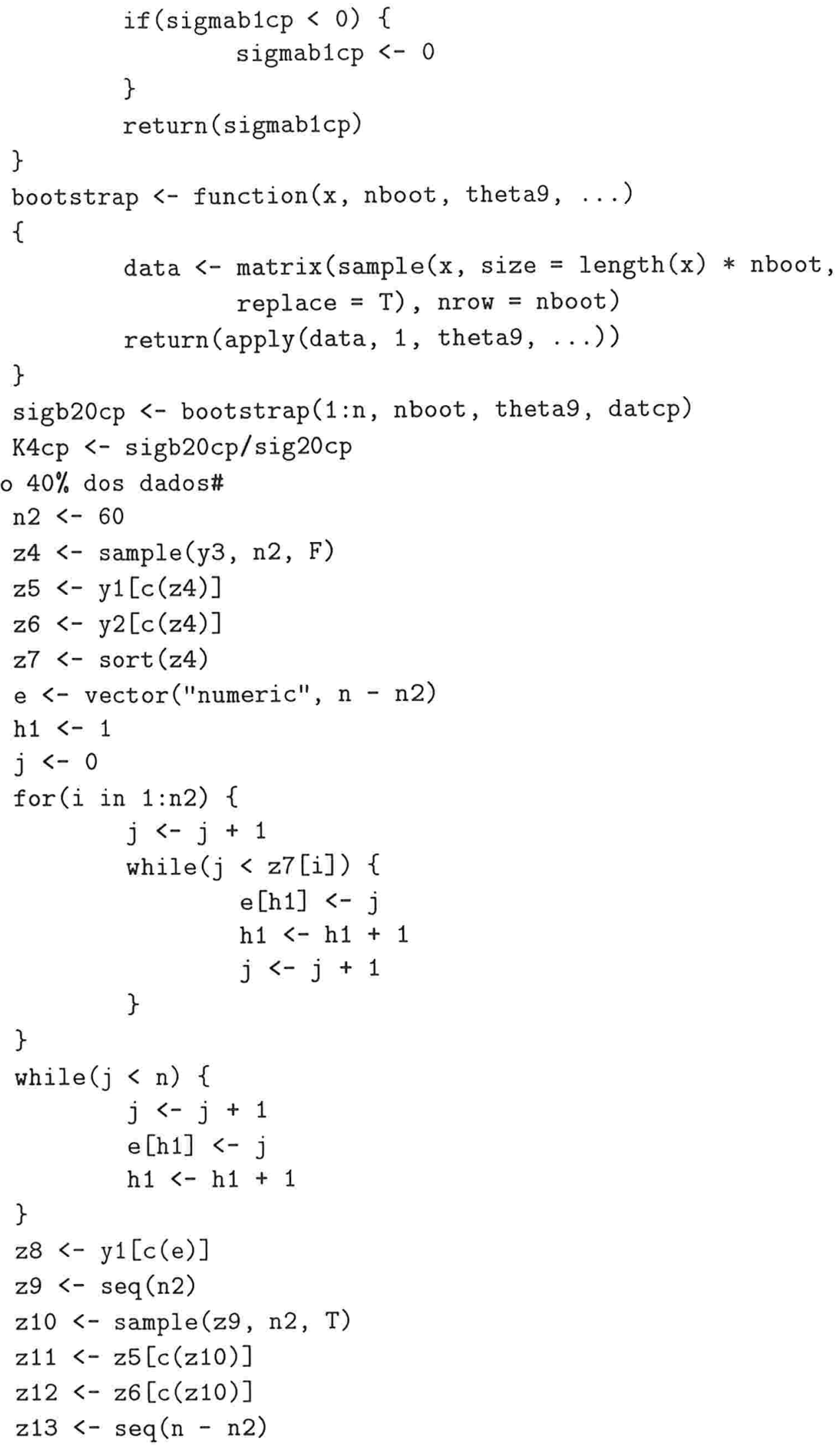




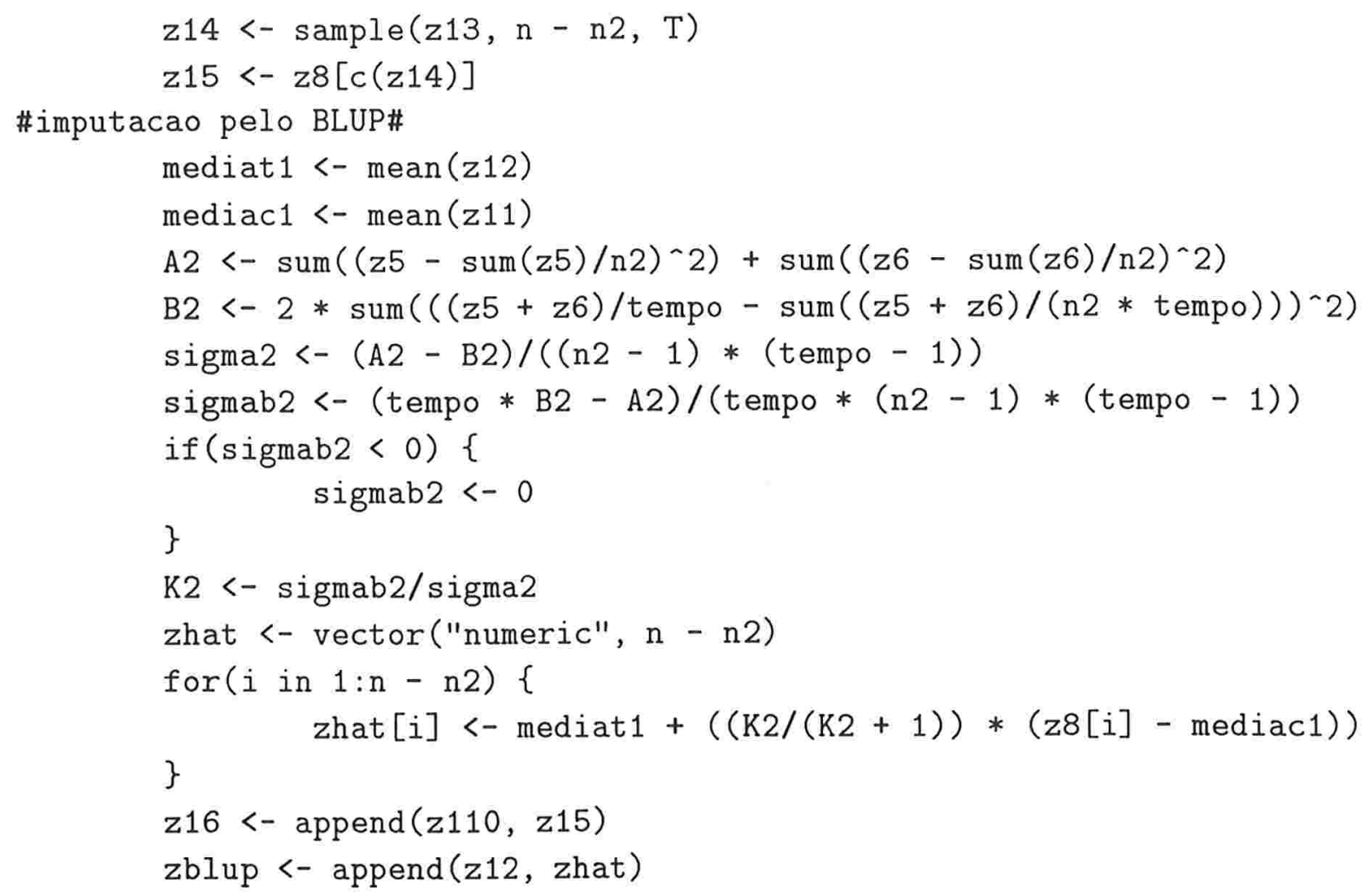

\#cálculo das variâncias usando bootstrap dos dados imputados pelo BLUP\# nboot $<-200$ 


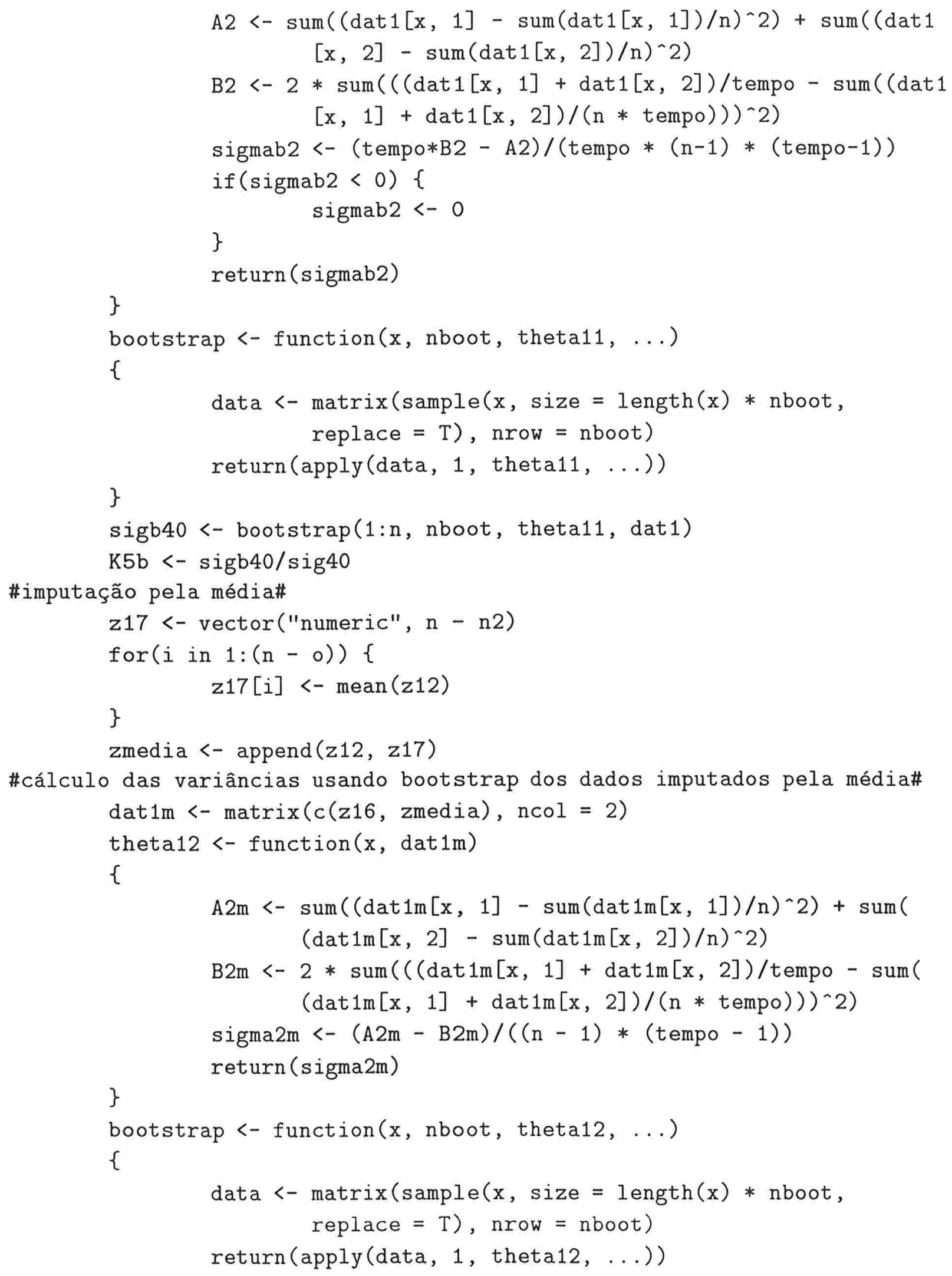




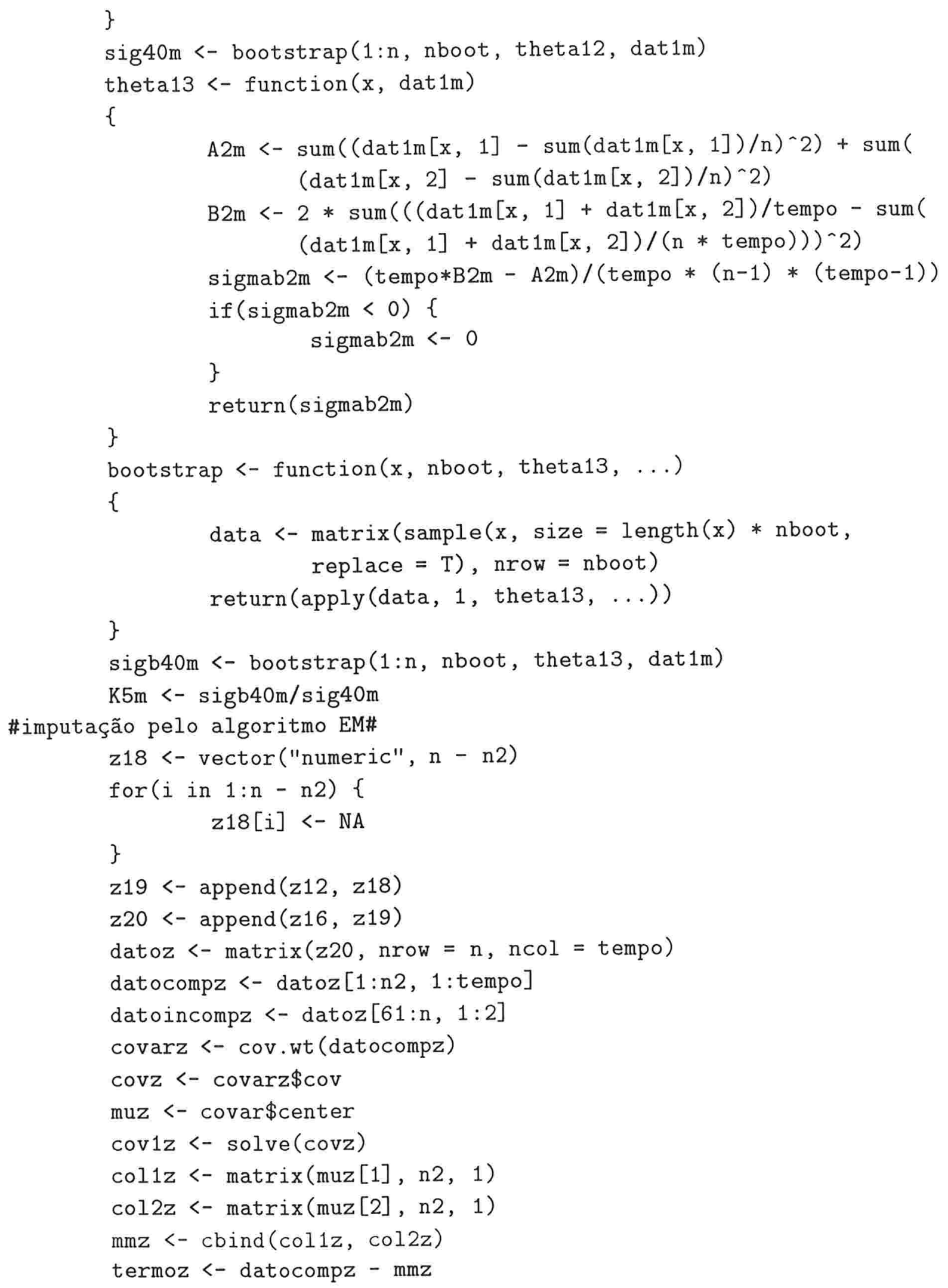




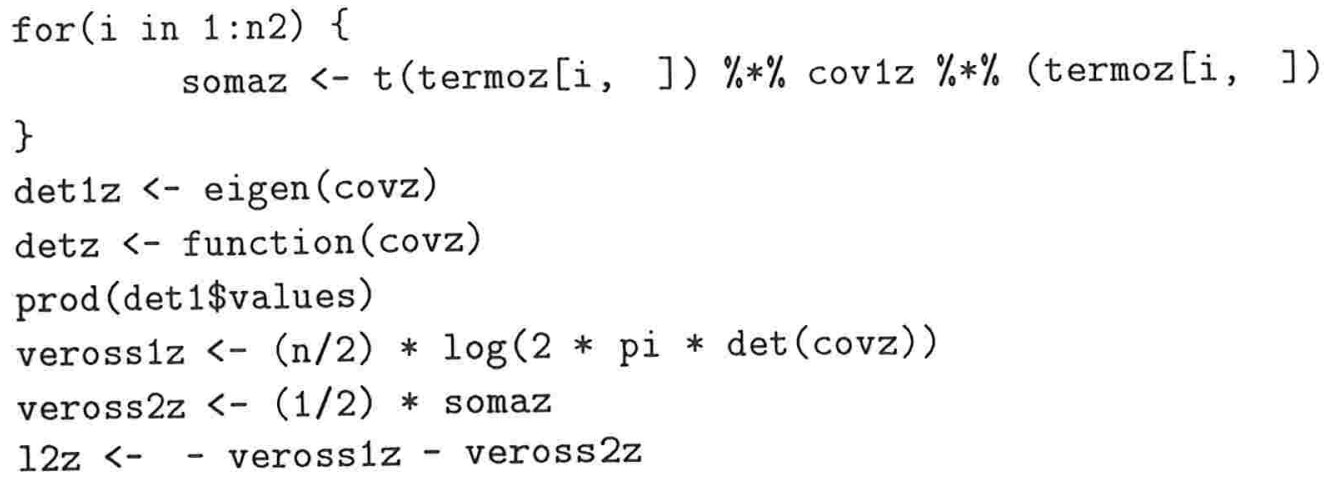

\#cálculo do vetor de médias e da matriz de covariâncias dos dados imputados\# covarz.c <- cov.wt (datosz)

covz.c <- covarz.c\$cov

muz.c <- covarz.c $\$$ center

cov1z.c <- solve (covz.c)

$\operatorname{col} 1 z . c<-\operatorname{matrix}(\operatorname{muz} . c[1], \mathrm{n}, 1)$

col2z.c <- matrix (muz.c[2], n, 1)

$\mathrm{mmz} . \mathrm{c}<-\operatorname{cbind}(\operatorname{col} 1 z . c, \operatorname{col} 2 z . c)$

termoz.c <- datosz - mmz.c

for ( $\mathrm{i}$ in $1: \mathrm{n})$ \{

somaz.c $<-\operatorname{sum}(t(t e r m o z . c[i]) \% * \,% \operatorname{cov} 1 z . c \% * \%$ (termoz.c $[i]$,$) )$

\}

$\operatorname{det} 2 z<-\operatorname{eigen}(\operatorname{covz} \cdot c)$

$\operatorname{detz}<-$ function (covz.c)

$\operatorname{prod}(\operatorname{det} 2 z \$$ values)

veross1z.c <- $(\mathrm{n} / 2) * \log (2 *$ pi $* \operatorname{detz}(\operatorname{covz} . \mathrm{c}))$

veross $2 z . c<-(1 / 2) *$ somaz.c

$11 \mathrm{z}<-\quad$ veross $1 z . c$ - veross $2 z . c$

c1 $<-1$

\#comparação das verossimilhanças\#

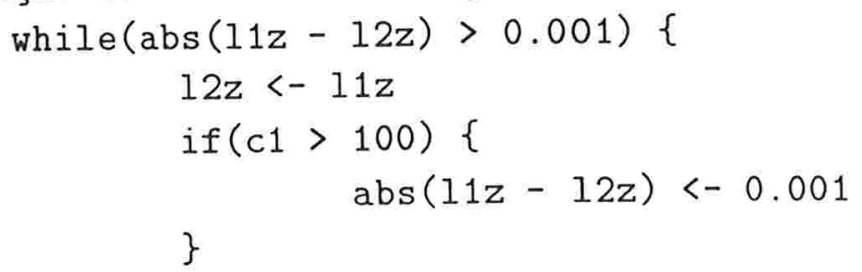




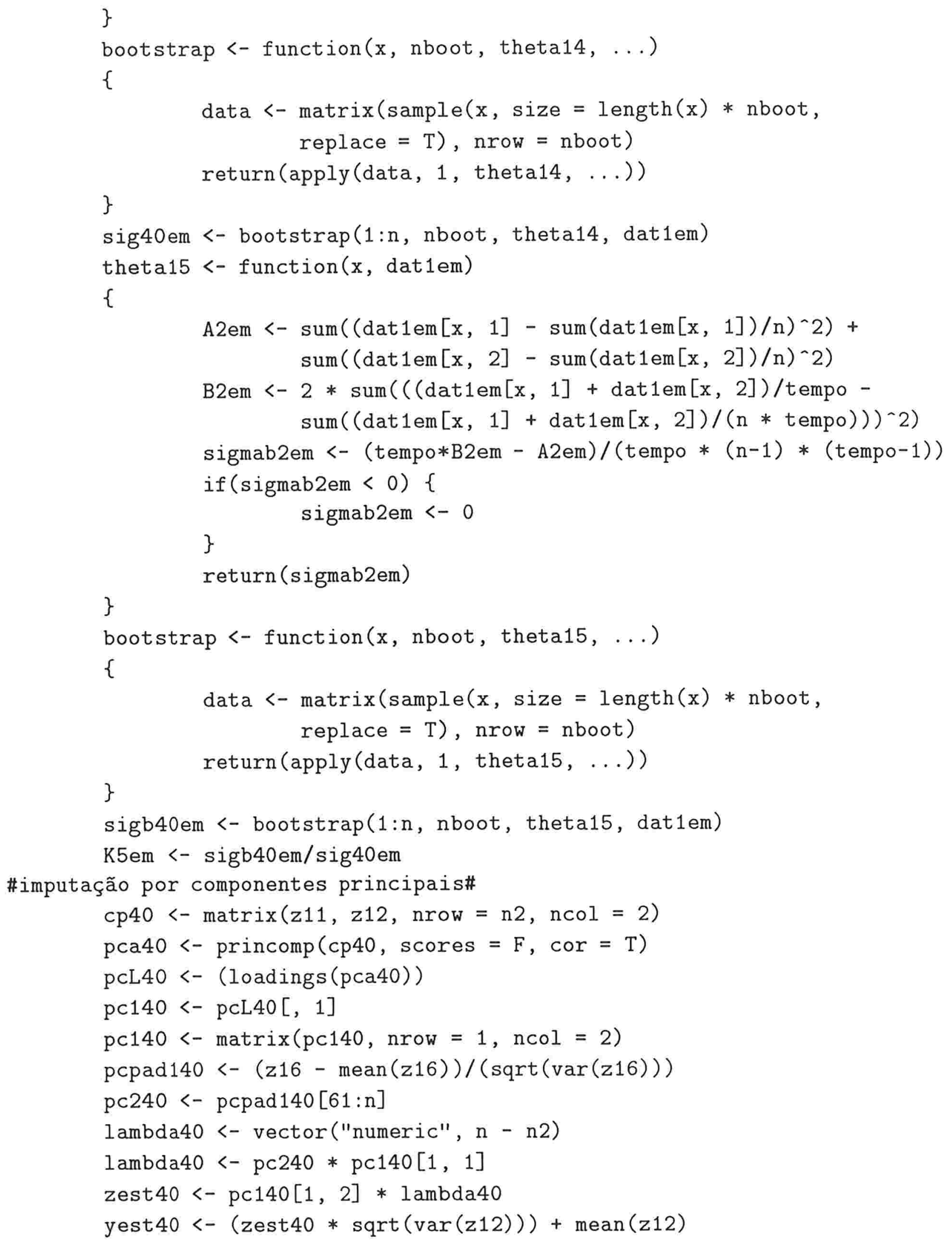




$$
z c p<- \text { append }(z 12 \text {, yest40) }
$$

\#cálculo das variâncias usando bootstrap dos dados imputados por componentes principais\#

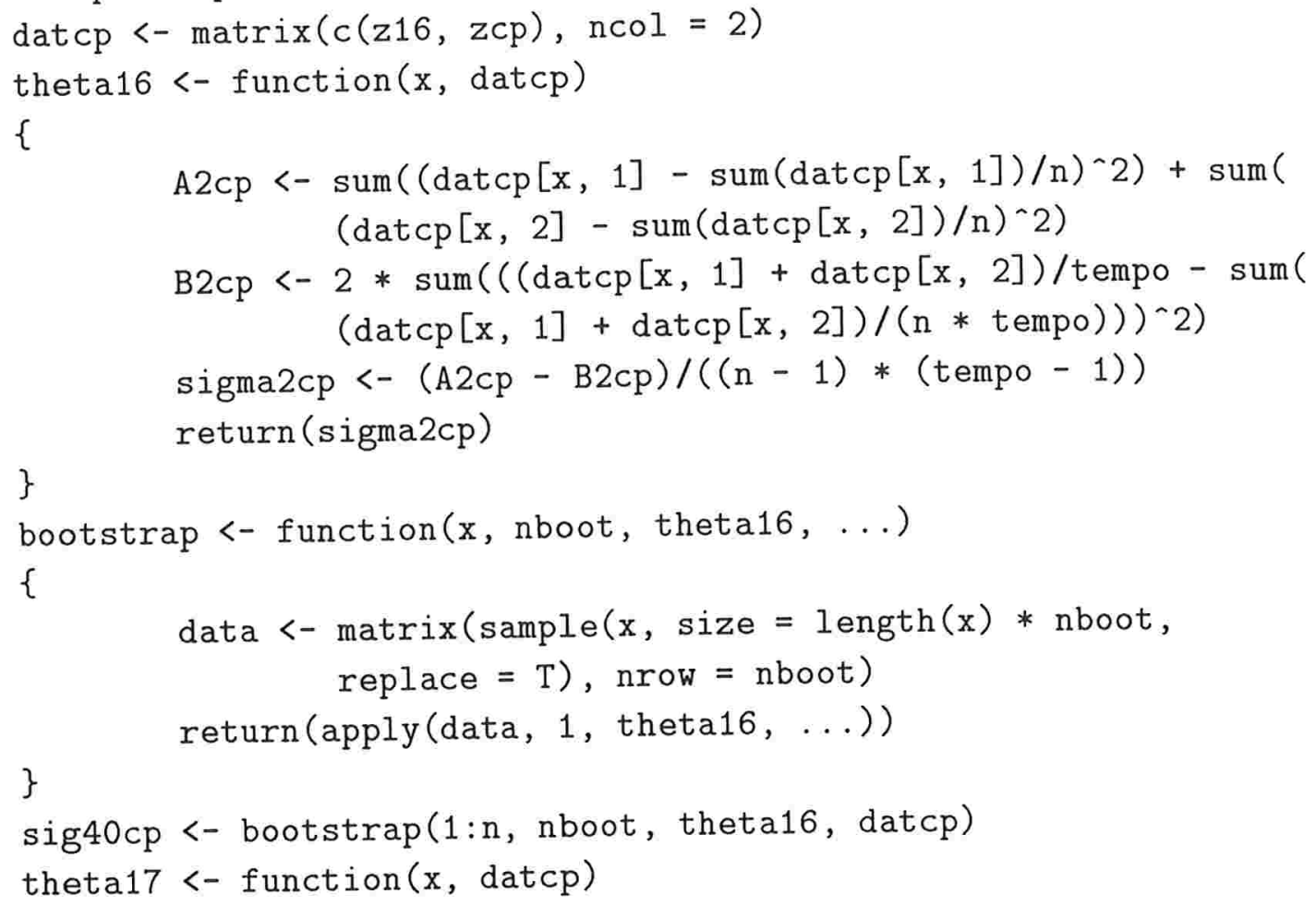


\#resultados dos parâmetros calculados das 2000 vezes\#

$$
\begin{aligned}
& \text { ww }[1]<- \text { ww }[1]+\text { sigma } \\
& w w[2]<-w w[2]+(\text { sigma }) \wedge 2 \\
& \text { ww [3] <- ww [3] + sigmab } \\
& \text { ww }[4]<- \text { ww }[4]+(\text { sigmab }) \wedge 2 \\
& \text { Ww }[5]<- \text { ww }[5]+K \\
& \text { ww }[6]<-w w[6]+K^{\wedge} 2 \\
& \text { ww }[7]<- \text { ww }[7]+\operatorname{var}(\text { sigma }) \\
& \text { ww }[8]<- \text { ww }[8]+(\operatorname{var}(\text { sigma }))^{\wedge} 2 \\
& \text { ww }[9]<- \text { ww }[9]+\operatorname{var}(\text { sigmab }) \\
& \text { ww }[10]<- \text { ww }[10]+(\operatorname{var}(\operatorname{sigmab})) \wedge 2 \\
& \text { ww }[11]<- \text { ww }[11]+\operatorname{mean}(\text { result }) \\
& \text { ww }[12]<- \text { ww }[12]+(\operatorname{mean}(\text { result })) \wedge 2 \\
& \text { ww [13] <- ww [13] + var (result) } \\
& \text { wW }[14]<- \text { ww }[14]+(\operatorname{var}(\text { result }))^{\wedge} 2 \\
& \text { ww }[15]<- \text { ww }[15]+\operatorname{mean}(\text { result } 1) \\
& W W[16]<-W W[16]+(\operatorname{mean}(\text { result1) }) \wedge 2 \\
& \text { ww }[17]<- \text { ww }[17]+\operatorname{var}(\text { result1) } \\
& \text { ww }[18]<- \text { ww }[18]+(\operatorname{var}(\operatorname{result1})) \wedge 2 \\
& \text { ww }[19]<- \text { ww }[19]+\operatorname{mean}(\text { K3 }) \\
& \text { ww }[20]<-w w[20]+(\operatorname{mean}(K 3))^{\wedge} 2 \\
& \text { ww [21]<- ww [21] + } \operatorname{var}(K 3) \\
& \text { Ww [22] <- ww [22] + }(\operatorname{var}(K 3))^{\wedge} 2 \\
& \text { Ww }[23]<- \text { ww }[23]+\operatorname{mean}(K 1) \\
& \text { ww }[24]<- \text { ww }[24]+(\operatorname{mean}(K 1)) \wedge 2 \\
& \text { ww [25] <- ww [25] + mean (sig20) } \\
& \text { Ww [26] <- ww [26] + (mean }(\operatorname{sig} 20))^{\wedge} 2 \\
& \text { ww }[27]<- \text { ww }[27]+\operatorname{var}(\operatorname{sig} 20) \\
& \text { WW }[28]<- \text { Ww }[28]+(\operatorname{var}(\operatorname{sig} 20))^{-} 2 \\
& \text { ww [29] <- ww [29] + mean (sigb20) } \\
& \text { ww }[30]<- \text { ww }[30]+(\text { mean }(\text { sigb20 }))^{\wedge} 2 \\
& \text { ww }[31]<-w w[31]+\operatorname{var}(\operatorname{sigb20}) \\
& \text { Ww [32]<- ww [32] + (var }(\operatorname{sigb20}))^{\wedge} 2 \\
& \text { ww [33] <- ww [33] + mean }(K 4 b) \\
& \text { ww [34] <- ww [34] + (mean }(K 4 b))^{\wedge} 2 \\
& \text { ww [35] <- ww[35] + } \operatorname{var}(K 4 b) \\
& \text { ww }[36]<-w w[36]+(\operatorname{var}(K 4 b)) \wedge 2 \\
& \text { ww }[37]<- \text { ww }[37]+\operatorname{mean}(\operatorname{sig} 20 \mathrm{~m}) \\
& \text { ww }[38]<- \text { ww }[38]+(\operatorname{mean}(\operatorname{sig} 20 \mathrm{~m}))^{\wedge} 2
\end{aligned}
$$




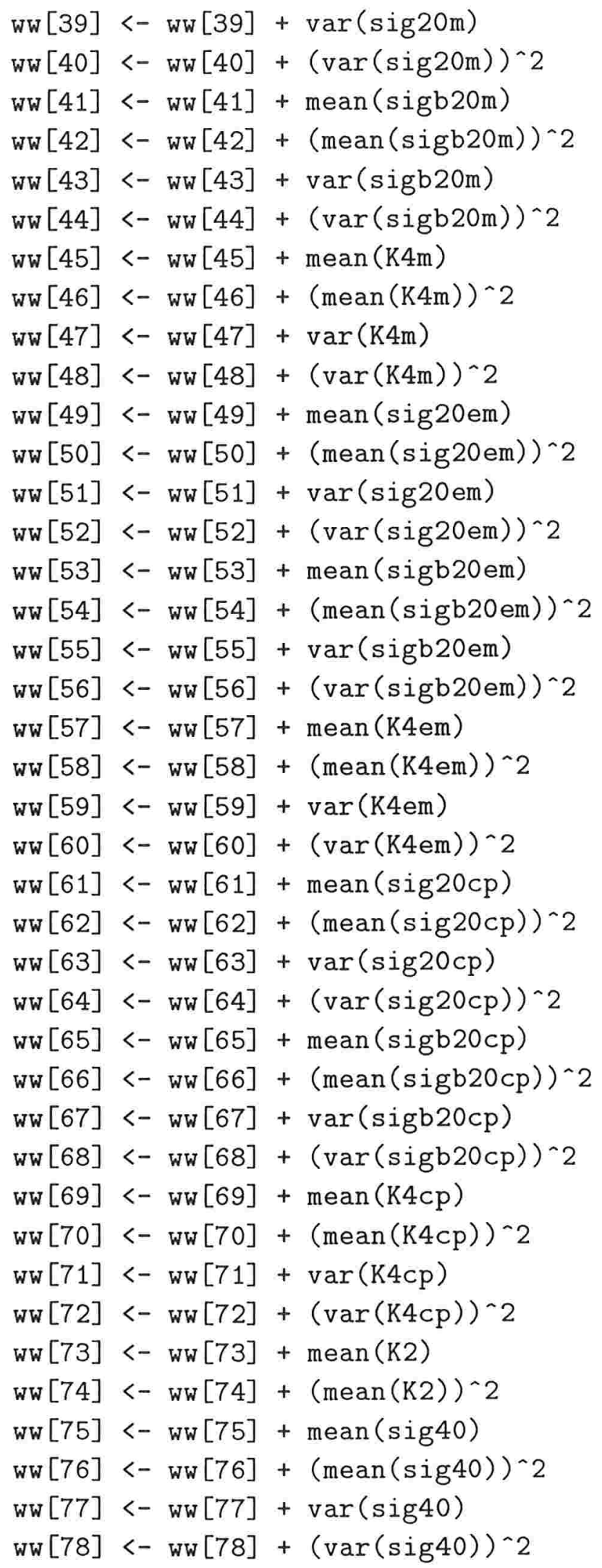




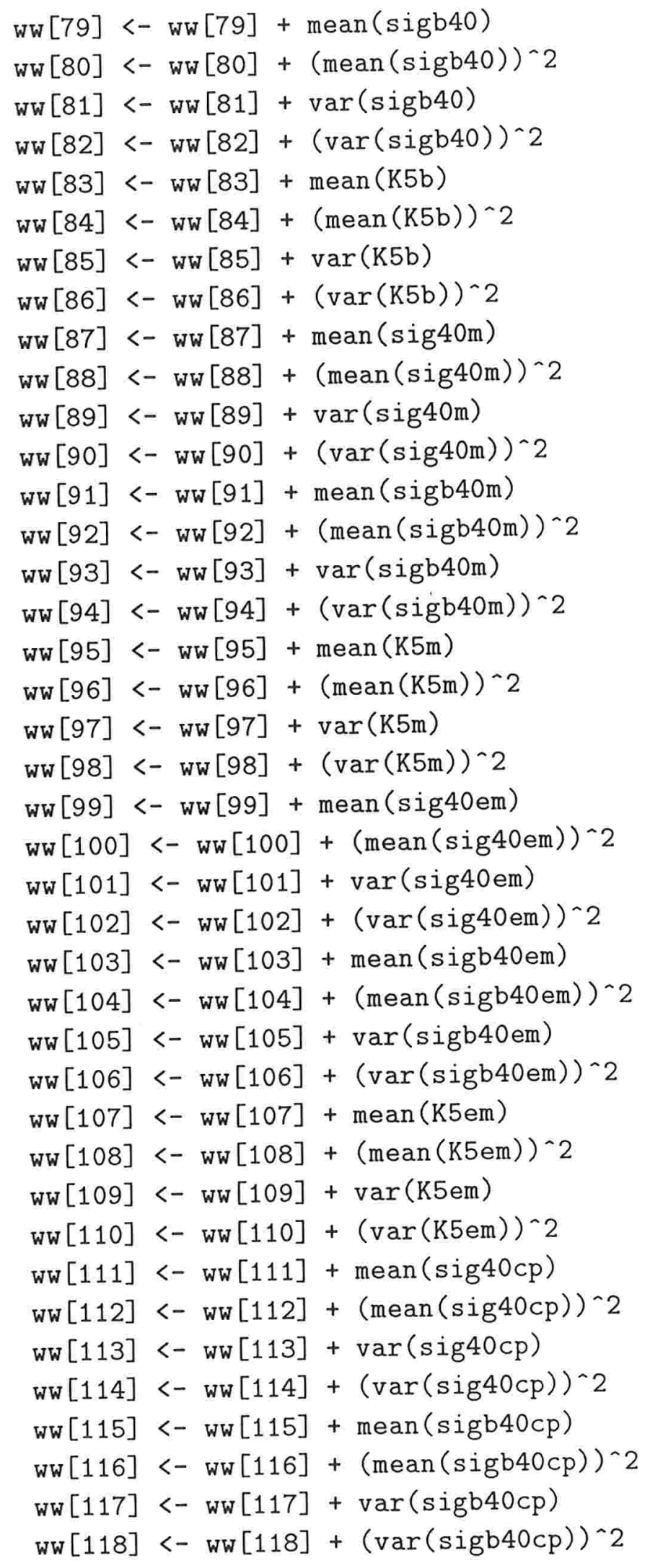




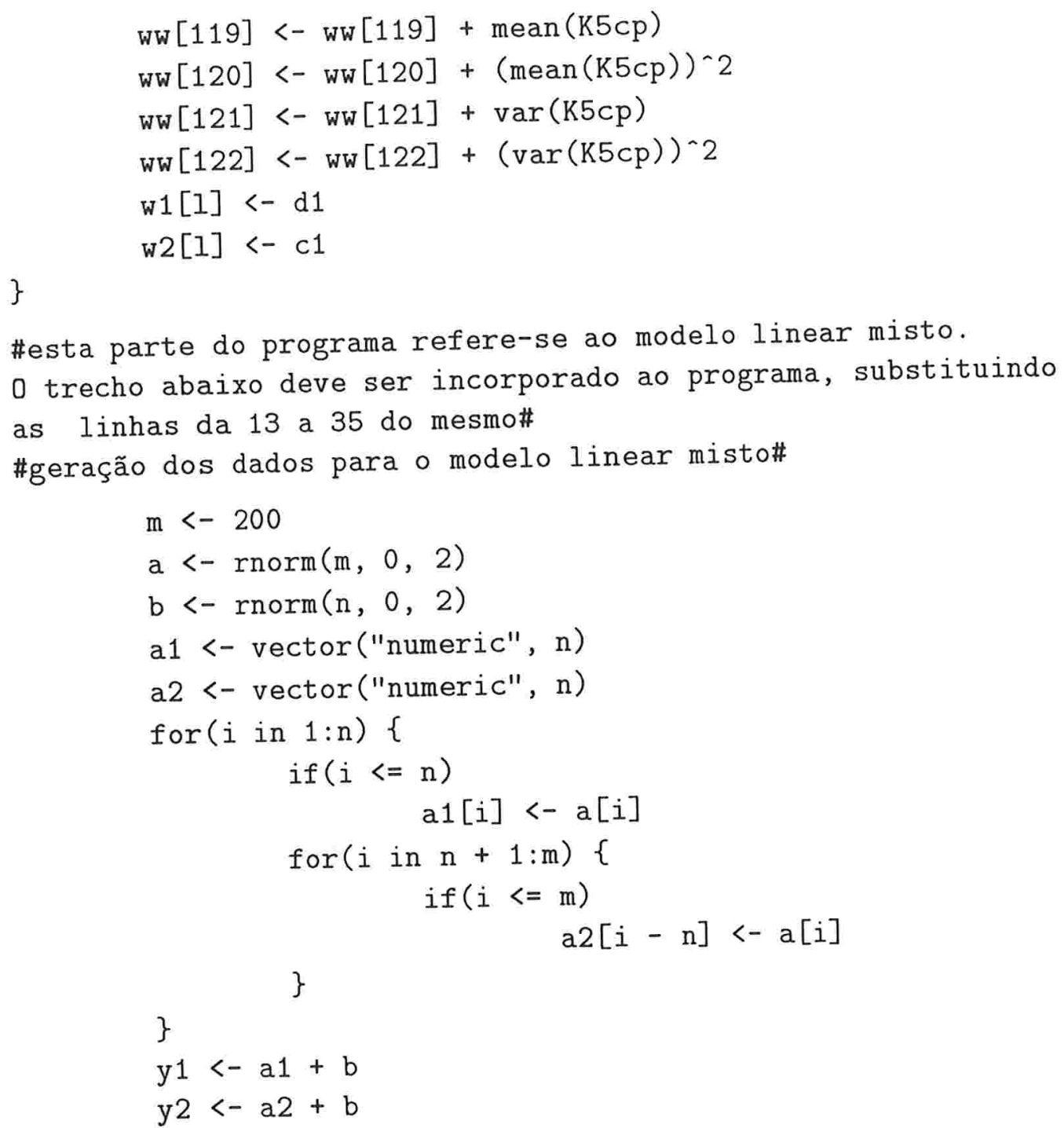




\section{Referências Bibliográficas}

[1] ALBIERI, S.(1989). A Ausência de Resposta em Pesquisas: uma Aplicação de Métodos de Imputação. Dissertação de Mestrado, IMPA-RJ.

[2] BARroso, L.P.(1995). Imputação de Dados em Painéis para Populações Finitas. Tese de Doutorado, Instituto de Matemáticas e Estatística - USP, São Paulo.

[3] BELLO, A.L.(1993). Choosing among imputation techniques for incomplete multivariate data: A simulation study. Comunications in Statistics A: Theory and Method 22 (3) 853-877.

[4] BELLO, A.L.(1994). A bootstrap Methods for Using Imputation Techniques for Data with Missing Values. Biometrics Journal 36 (4) 453-464.

[5] BERAN, R. and SRIVASTAVA, M.S. (1985). Bootstrap tests and confidence regions for functions of a covariance matrix. The Annals of Statistics, 13 (1), 95-115.

[6] BUSSAB, W.O and MORETTIN, P.A.(1991). Métodos Quantitativos - Estatística Básica. Atual Editora.

[7] DAVISON,A.C. and HINKLEY, D.V. (1992). Computer-intensive Statistical methods. In Y. Dodge and J. Whittaker (eds), COMPSTAT 1992. Physica Verlag, 2 51-62.

[8] DEAR, R.E.(1959). A principal component missing data method for multiple regression models, report SP-86. System- Development Corporation. Santa Mónica, CA.

[9] DEMPSTER, A.P.; LAIRD, N.M. and RUBIN, D.B. (1977). Maximum likelihood estimation from incomplet data via the EM algorithm (with discussion). Journal of the Royal Statistical Society, Series, B, 39, 1-38. 
[10] DICICCIO, T.J. and ROMANO,J.P. (1988). A Review of bootstrap confidence intervals. Journal of the Royal Statistical Society, Series, B, 50 (3), 338-354.

[11] DICICCIO, T.J. and EFRON, B. (1996). Bootstrap Confidence Intervals. Statistical Science, 11 (3), 189-228.

[12] EFRON, B. (1979). Bootstrap Methods: Another Look at the Jackknife. The Annals of Statistics, 7 (1), 1-26.

[13] EFRON, B. (1982). The Jackknife, the Bootstrap, and other resampling plans. Soc. Ind. Appl. Math. CBSM Natl Sci. Found Monograph, 38.

[14] EFRON, B. (1987). Better Bootstrap Confidence Intervals. Journal of the American Statistical Association, 82, 171-185.

[15] EFRON, B. (1990). More Efficient Bootstrap Computations. Journal of the American Statistical Association, 85 (409), 79-89.

[16] EFRON, B. (1994). Missing Data, Imputation, and the Bootstrap. Journal of the American Statistical Association, 89 (426), 463-479.

[17] EFRON, B. and TIBSHIRANI, R. (1986). Bootstrap Methods for Standard Error, Confidence Intervals, and Other Measures of Statistical Accuracy. Statistical Science, 1, 54-77.

[18] EFRON, B. and TIBSHIRANI, R. (1993). An Introduction to the Bootstrap. Chapman and Hall. New York.

[19] FISHER N.I. and HALL P. (1989). Bootstrap Confidence Regions for Directional Data. Journal of the American Statistical Association, 84 (408), 996-1002.

[20] FRANGOS,C.C. and SCHUCANYW.R. (1995). Improved bootstrap confidence intervals in certain toxicological experiments. Comunications in Statistics A: Theory and Method 24 829-844.

[21] FREEDMAN, D.A. (1981). Bootstrapping regression models. The Annals of Statistics, 9 (61), 1218-1228.

[22] HALL, P. (1988). On symmetric bootstrap confidence intervals. Journal of the Royal Statistical Society, Series, B, 50 (1), 35-45.

[23] HAMMERSLEY. I.M. and HANDSCOMB, D.C. (1964). Monte Carlo Methods, John Wiley and Sons. N.Y 
[24] HENDERSON, C.R. (1950). Estimation of genetic parameters. The Annals of Mathematical Statistics, 21, 309-310.

[25] HENDERSON, C.R. (1953). Estimation of variance and covariance components. Biometrics, 19, 226-252.

[26] HENDERSON, C.R. (1975). Best linear unbiased estimation and prediction under a selection model. Biometrics, 31, 423-447.

[27] JOHNS, M.V. (1988). Importance sampling for bootstrap confidence intervals. Journal of the American Statistical Association, 83 (403), 709-714.

[28] LITTLE, R.J.A. and RUBIN, D.B. (1987). Statistical analysis with Missing Data, Wiley NY.

[29] LO, A.Y. (1987). A large-sample study of the Bayesian bootstrap. The Annals of Statistics, 15 (1), 360-375.

[30] MANTEIGA, W.G. e SÁNCHEZ, J.M. (1994). The bootstrap - A Review. Computational Statistics, 9, 165-205.

[31] QUEnouille, M. (1949). Aproximate Tests of Correlation in Time Series. Journal of the Royal Statistical Society, Series, B, 11, 18-84.

[32] ROBINSON, G.K. (1991). That BLUP is a good thing: the estimation of random effects. Statistical Sciencie, 6, 15-51.

[33] RUBIN, D.B. (1976). Inference and missing data. Biometrika, 63, 581-592.

[34] RUBIN, D.B. (1978). Multiple Imputation in Sample Surveys - A Phenomenological Bayesian Approach to Nonresponse. Proceedings of the Survey Research Methods Section, American Statistical Association, 20-34.

[35] RUBIN, D.B. (1981). The Bayesian bootstrap. The Annals of Statistics, 9 (1), 130-139.

[36] RUBIN, D.B. (1987). Multiple Imputation for Nonresponse in Surveys, Wiley, NY.

[37] SEARLE, S.R. (1971). Linear Models, John Wiley and Sons, N.Y.

[38] SILVA, P.L.N. (1989). Crítica e Imputação de Dados Quantitativos Utilizando o SAS. Dissertação de Mestrado, IMPA-RJ. 
[39] SHAO, J. and SITTER, R.R. (1996). Bootstrap for Imputed Survey Data. Journal of the American Statistical Association, 91 (435), 1278-1288.

[40] SITTER, R.R. (1992). Comparing three Bootstrap Methods for Survey Data. Canadian Journal of Statistic, 20, 134-154.

[41] WILKS, S.S. (1932). Moments and Distribution of estimates of population parameters from fragmentary samples. The Annals of Statistics, 3, 163-195.

[42] WU. C.F.J. (1986). Jackknife, bootstrap and another resampling methods in regression analysis (with discussion). The Annals of Statistics, 14 (4), 1261-1295.

[43] YOUNG, G. A. (1994). Bootstrap: More than a Stab in the Dark? Statistical Science, 9 (3), 382-415. 